\title{
COHOMOLOGY OF FINITE GROUP SCHEMES OVER A FIELD
}

\author{
Eric M. Friedlander* ANd Andrei Suslin**
}

A finite group scheme $G$ over a field $k$ is equivalent to its coordinate algebra, a finite dimensional commutative Hopf algebra $k[G]$ over $k$. In many contexts, it is natural to consider the rational (or Hochschild) cohomology of $G$ with coefficients in a $k[G]$-comodule $M$. This is naturally isomorphic to the cohomology of the dual cocommutative Hopf algebra $k[G]^{\#}$ with coefficients in the $k[G]^{\#}$-module $M$. In this latter formulation, we encounter familiar examples of the cohomology of group algebras $k \pi$ of a finite groups $\pi$ and of restricted enveloping algebras $V(g)$ of finite dimensional restricted Lie algebras $g$.

In recent years, the representation theory of the algebras $k \pi$ and $V(g)$ has been studied by considering the spectrum of the cohomology algebra with coefficients in the ground field $k$ and the support in this spectrum of the cohomology with coefficients in various modules. This approach relies on the fact that $H^{*}(\pi, k)$ and $H^{*}(V(g), k)$ are finitely generated $k$-algebras as proved in [G], [E], [V], [FP2]. Rational representations of algebraic groups in positive characteristic correspond to representations of a hierarchy of finite group schemes. In order to begin the process of introducing geometric methods to the study of these other group schemes, finite generation must be proved. Such a proof has proved surprisingly elusive (though partial results can be found in [FP2]).

The main theorem of this paper is the following:

Theorem 1.1. Let $G$ be a finite group scheme and $M$ a finite dimensional rational $G$-module. Then $H^{*}(G, k)$ is a finitely generated $k$-algebra and $H^{*}(G, M)$ is a finite $H^{*}(G, k)$-module.

Work in progress by C. Bendel (and the authors) reveals that Theorem 1.1 and its proof will provide interesting theorems of a geometric nature concerning the representation theory of finite group schemes.

In a sense that is made explicit in section 1, our proof of finite generation is quite constructive. We embed $G$ in some general linear group $G L_{n}$ and establish the existence of universal extension classes for $G L_{n}$ of specified degrees. In a direct manner, these classes provide the generators of $H^{*}(G, k)$.

In order to construct these universal extension classes, we follow closely the approach of V. Franjou, J. Lannes, and L. Schwartz [FLS]. This entails the study of extensions in a certain category of functors (in our context, "polynomial functors"). Certain aspects of this category enable computations of Ext-groups which seem inaccessible when studying the Ext-groups of $G$-comodules. On the other hand,

*Partially supported by the N.S.F. and N.S.A. grant \# MDA 904-93-H-3029

**Partially supported by the I.S.F. grant 0100 and the N.S.F. grant DMS - 9510242

Typeset by $\mathcal{A} \mathcal{M S}-\mathrm{T}_{\mathrm{E}} \mathrm{X}$ 
our functor category is closely related to the category of modules over classical Schur algebras as shown in section 3 .

Much work must be done after constructing these universal classes to establish that they have the requisite good properties. This work entails further computations of Ext-groups in the category of polynomial functors.

In section 7 , we provide as a corollary of our analysis a new proof of a theorem of M. Bökstedt[B] computing the homology $H_{*}\left(G L\left(\mathbf{F}_{q}\right), M\left(\mathbf{F}_{q}\right)\right)$ of the infinite general linear group over a finite field $\mathbf{F}_{q}$ with ceofficients in its adjoint representation. We find this pleasing, for our proof is purely algebraic and does not refer to results in geometric topology.

Except for fields of characteristic 2, we have not found explicit extensions representing our universal extension classes. This is one of the many challenges which remain. The ambitious reader is encouraged to try his hand at further calculations of functor cohomology which may have bearing on computations of certain algebraic K-groups.

We briefly summarize the contents of this paper. In $\S 1$, we show that Theorem 1.1 is implied by the existence of certain universal extension classes for general linear groups over fields of positive characteristic $p$. The universality of these classes suggest that they should have independent interest. Our category $\mathcal{P}$ of polynomial functors of finite degree is introduced in $\S 2$ and its basic properties are proved. These include the existence of explicit projective and injective generators and a vanishing result crucial for our calculations of Ext-groups. In a somewhat different guise, this vanishing theorem was first proved by M. Jibladze and T. Pirashvili [JP]. The relationship between polynomial functors of finite degree and modules over classical Schur algebras is made explicit in $\S 3$ using the properties of $\mathcal{P}$ established in $\S 2$. Following S. Donkin $[\mathrm{D}]$, we also give a proof of the close relation between cohomology of Schur algebras and rational cohomology of general linear groups.

In $\S 4$, we present a somewhat shortened version of the ingenious constructions of Franjou-Lannes-Schwartz which establishes the existence of our fundamental extensions. The technique which we have borrowed is the exploitation through hypercohomology spectral sequences of the complexes of DeRham and Koszul. Necessary further computations of Ext-groups between polynomial functors are provided in $\S 5$, enabling us to identify in $\S 6$ the restrictions of our universal classes to certain infinitesimal sub-group schemes of $G L_{n}$. At this point, the proof of Theorem 1.1 is complete. We continue the paper with our computation of $H_{*}\left(G L\left(\mathbf{F}_{q}\right), M\left(\mathbf{F}_{q}\right)\right)$ in $\S 7$. Finally, in $\S 8$ we construct (in the special case of fields of characteristic 2) explicit injective resolutions of functors $S^{m(r)}$ which give immediate computations of the corresponding Ext-groups and provide, in particular, representatives for our universal extensions $e_{r}$.

As pointed out to us by L. Avramov, the finite generation of the cohomology of a finite dimensional commutative Hopf algebra $A$ follows easily from the known structure of such algebras as a semi-tensor product of a separable algebra and a polynomial algebra truncated by $p$-primary powers of the indeterminants, where $p$ is the characteristic of the ground field (cf. [W]). We do not know whether it is reasonable to expect finite generation of the cohomology of an arbitrary finite dimensional Hopf algebra.

The first author is indebted to many friends for their help and encouragement 
during the long evolution of this proof of finite generation. Brian Parshall, especially, provided many insights and detected many errors in earlier efforts; Bill Dwyer recommended consideration of [FLS], Len Evens helped us understand the role of finite groups, and Dan Nakano pointed out S. Donkin's work [D] on Schur algebras.

\section{§1. Reduction to the existence of universal Classes}

We show that Theorem 1.1 as restated below is implied by the existence of certain universal classes in the rational cohomology of the general linear group.

Let $k$ be a field. We denote by $G L_{n}=G L_{n, k}$ the general linear group over $k$, i.e.

$$
G L_{n, k}=\operatorname{Spec}\left(k\left[\left(X_{i j}\right)_{i, j=1}^{n}, t\right] /\left(\operatorname{det}\left(X_{i j}\right) \cdot t-1\right)\right) .
$$

An affine group scheme over $k$ is an affine scheme of finite type over $k$ endowed with a structure of group scheme over $k$. It's well known (see [W]) that any affine group scheme over $k$ may be embeded as a closed subgroup scheme into some $G L_{n}$. The affine group scheme $G / k$ is said to be a finite group scheme if the coordinate ring $k[G]$ is a finite dimensional $k$-vector space, or (what's the same) if $G$ as a scheme consists of finitely many (closed) points.

Note that for any affine group scheme $G$ the coordinate ring $k[G]$ has a natural structure of a Hopf algebra. We recall that a rational $G$-module $M$ is a comodule for the coalgebra $k[G]$. If $M$ is finite dimensional over $k$, then to make $M$ into a rational $G$-module is the same as to give a $k$-linear action

$$
G \times M \rightarrow M
$$

where we use the same notation $M$ for the corresponding scheme $\operatorname{Spec} S^{*}\left(M^{\#}\right)$ (here $M^{\#}$ stands for the dual vector space and $S^{*}$ denotes the symmetric algebra). This is also the same as to give a homomorphism of group schemes over $k$

$$
G \rightarrow G L(M)
$$

where $G L(M)$ is the open subscheme of $\operatorname{End}_{k}(M)$, consisting of endomorphisms with non-zero determinant (i.e. $G L(M) \cong G L_{n, k}$ provided that $\operatorname{dim}_{k} M=n$ ). For a rational $G$-module $M$ we denote by $H^{*}(G, M)$ the corresponding rational cohomology groups, defined as the Ext-groups $\operatorname{Ext}^{*}(k, M)$ in the category of rational $G$-modules (see $[\mathrm{J}]$ ).

Theorem 1.1. Let $G$ be a finite group scheme over $k$ and let $M$ be a finite dimensional rational $G$-module. Then $H^{*}(G, k)$ is a finitely generated $k$-algebra and $H^{*}(G, M)$ is a finite $H^{*}(G, k)$-module.

This theorem is trivial if char $k=0$ (in this case $H^{i}(G, M)=0$ for $i>0$ and any rational $G$-module $M)$. So from now on we assume that char $k=p>0$. We first recall the definition and elementary properties of the Frobenius twist.

Denote by $f: k \rightarrow k$ the field embeding given by the formula $f(\lambda)=\lambda^{p}$. For any vector space $M$ over $k$ we denote by $M^{(1)}$ the new vector space obtained from $M$ by means of the base change $f: k \rightarrow k$

$$
M^{(1)}=M \otimes_{f} k \text {. }
$$


We shall use the notation $m^{(1)}$ for the element $m \otimes_{f} 1 \in M^{(1)}$. Thus $M^{(1)}$ is generated as a $k$-vector space by elements $m^{(1)}$ and the only relations on these generators are of the form

$$
\left(m_{1}+m_{2}\right)^{(1)}=m_{1}^{(1)}+m_{2}^{(1)} ; \quad(\lambda m)^{(1)}=\lambda^{p} \cdot m^{(1)} .
$$

Note that $M \mapsto M^{(1)}$ is an exact additive functor (called the Frobenius twist) from the category of $k$-vector spaces to itself. As any base change functor, the Frobenius twist takes tensor products to tensor products and duals (of finite dimensional spaces) to duals, i.e. we have natural isomorphisms

$$
\begin{aligned}
(M \otimes N)^{(1)} & \stackrel{\sim}{\longrightarrow} M^{(1)} \otimes N^{(1)} \\
\left(M^{\#}\right)^{(1)} & \stackrel{\sim}{\longrightarrow}\left(M^{(1)}\right)^{\#} \quad\left(\operatorname{dim}_{k} M<\infty\right) .
\end{aligned}
$$

In particular, if $M$ happens to be a $k$-algebra ( $k$-coalgebra, Hopf algebra over $k, \ldots)$ then $M^{(1)}$ is naturally endowed with the same kind of structure.

Let $A$ be a commutative $k$-algebra. According to what was said above, in this case $A^{(1)}$ is also a commutative $k$-algebra. Moreover we have a natural homomorphism of $k$-algebras $A^{(1)} \rightarrow A: a \otimes \lambda \mapsto \lambda \cdot a^{p}$. For any $k$-vector space $M$ we get, applying the previous remark to the $k$ algebra $A=S^{*}(M)$, a natural homomorphism of $k$-vector spaces $M^{(1)} \rightarrow S^{p}(M): m \otimes \lambda \mapsto \lambda \cdot m^{p}$.

The above construction admits an immediate globalization. For any scheme $X /$ Spec $k$ we set $X^{(1)}=X \otimes_{f} k$. As in the affine case, we have a natural isomorphism of schemes over $k:\left(X \times_{k} Y\right)^{(1)} \stackrel{\sim}{\longrightarrow} X^{(1)} \times_{k} Y^{(1)}$. This shows, in particular, that for a group scheme $G / k$ the scheme $G^{(1)} / k$ also has a natural group structure. Furthermore, for any scheme $X / k$ we have a natural morphism of schemes over $k \quad F=F_{X}: X \rightarrow X^{(1)}$, called the Frobenius morphism (in the affine case, this morphism corresponds to the $k$-algebra homomorphism $A^{(1)} \rightarrow A$ ). If $G / k$ is a group scheme, then one checks easily that the Frobenius morphism $F_{G}: G \rightarrow G^{(1)}$ is a group homomorphism.

Let $G / k$ be an affine group scheme and let $M$ be a rational $G$-module. Applying the Frobenius twist to the structure homomorphism $M \rightarrow M \otimes k[G]$ we get a homomorphism $M^{(1)} \rightarrow(M \otimes k[G])^{(1)}=M^{(1)} \otimes k\left[G^{(1)}\right]$, making $M^{(1)}$ a rational module over the affine group scheme $G^{(1)}$. Using next the natural homomorphism of group schemes $F_{G}: G \rightarrow G^{(1)}$ we make further $M^{(1)}$ into a rational $G$-module. Thus Frobenius twist defines a functor from the category of rational $G$-modules to itself.

Assume that the $k$-vector space $M$ is defined over the prime field $\mathbb{F}_{p}$ (i.e. we are given an $\mathbb{F}_{p}$-vector space $M_{0}$ and an isomorphism $\left.M_{0} \otimes_{\mathbb{F}_{p}} k \stackrel{\sim}{\longrightarrow} M\right)$. In this case we have a natural isomorphism $M^{(1)}=\left(M_{0} \otimes_{\mathbb{F}_{p}} k\right) \otimes_{f} k=M_{0} \otimes_{\mathbb{F}_{p}} k=M$. In the same way if a scheme $X / k$ is defined over $\mathbb{F}_{p}$ then we get a natural isomorphism of schemes over $k X^{(1)} \cong X$. Furthermore if $G / k$ is a group scheme defined over $\mathbb{F}_{p}$ then the corresponding isomorphism $G^{(1)} \cong G$ is compatible with the group structures on $G$ and $G^{(1)}$ respectively. Thus for group schemes defined over $\mathbb{F}_{p}$, the Frobenius homomorphism may be written in the form

$$
F: G \rightarrow G
$$


According to definitions, this homomorphism corresponds to the Hopf algebra homomorphism $k[G] \rightarrow k[G]$ which is identity on $k$ and coincides with raising to the power $p$ on $\mathbb{F}_{p}\left[G_{0}\right]$. We denote by $G_{(r)}$ the kernel of the iterated Frobenius homomorphism $F^{r}: G \rightarrow G$. Thus, in particular,

$$
\left(G L_{n, k}\right)_{(r)}=\operatorname{Spec} k\left[\left(X_{i j}\right)_{i, j=1}^{n}\right] /\left(X_{i j}^{p^{r}}-\delta_{i j}\right)
$$

One reason why Frobenius twist behaves well with respect to the group actions lies in the fact that it is a polynomial functor. By this we mean that for any finite dimensional $k$-vector spaces $M, N$ the map $\operatorname{Hom}_{k}(M, N) \rightarrow \operatorname{Hom}_{k}\left(M^{(1)}, N^{(1)}\right)$ defined by the Frobenius twist is a homogeneous polynomial map of degree $p$. This fact may be used to give a slightly different description of the $G$-structure on $M^{(1)}$. Assume that $M$ is a finite dimensional rational $G$-module. The polynomial map $\operatorname{End}_{k}(M) \rightarrow \operatorname{End}_{k}\left(M^{(1)}\right)$ may be considered as a morphism of schemes. Furthermore this morphism of schemes takes the open subscheme $G L(M) \subset \operatorname{End}_{k}(M)$ to $G L\left(M^{(1)}\right) \subset \operatorname{End}_{k}\left(M^{(1)}\right)$ and defines a group scheme homomorphism

$$
G L(M) \rightarrow G L\left(M^{(1)}\right)\left(=(G L(M))^{(1)}\right) .
$$

Composing this homomorphism with the original homomorphism $G \rightarrow G L(M)$ we get a homomorphism $G \rightarrow G L\left(M^{(1)}\right)$ making $M^{(1)}$ into a rational $G$-module. One checks immediately that this $G$-structure coincides with the one discussed above.

We proceed to show that our Theorem 1.1 is implied by the following result, in the formulation of which we denote by $g l_{n}$ the adjoint representation of $G L_{n}$ (viewed as a rational $G L_{n}$-module).

Theorem 1.2. Assume that $k$ is a field of characteristic $p>0$. For any $n>1$, $r \geq 1$ there exist rational cohomology classes (defined over the prime field $\mathbb{F}_{p}$ )

$$
e_{r} \in H^{2 p^{r-1}}\left(G L_{n, k}, g l_{n}^{(r)}\right)
$$

which restrict nontrivially to $H^{2 p^{r-1}}\left(\left(G L_{n, k}\right)_{(1)}, g l_{n}^{(r)}\right)=H^{2 p^{r-1}}\left(\left(G L_{n, k}\right)_{(1)}, k\right) \otimes$ $g l_{n}^{(r)}$.

Proof. We shall construct the classes $e_{r}$ in section 4 and establish the non-triviality of their restriction to $\left(G L_{n, k}\right)_{(1)}$ in section 6 .

Remark 1.2.1. The classes $e_{r}$ defined for all $n>1$ are compatible in the following sense. For any $n \leq m$ we consider the standard embeding (into the left upper corner)

$$
i=i_{n, m}: G L_{n, k} \hookrightarrow G L_{m, k}
$$

and the standard $G L_{n, k}$-equivariant projection

$$
p=p_{n, m}: g l_{m} \rightarrow g l_{n}
$$

sending the matrix $\alpha=\left(a_{i j}\right)_{i, j=1}^{m}$ to its left upper corner $p(\alpha)=\left(a_{i j}\right)_{i, j=1}^{n}$. The pair $(i, p)$ defines homomorphisms on the rational cohomology groups

$$
H^{*}\left(G L_{m, k}, g l_{m}^{(r)}\right) \stackrel{i^{*}}{\longrightarrow} H^{*}\left(G L_{n, k}, g l_{m}^{(r)}\right) \stackrel{\left(p^{(r)}\right)_{*}}{\longrightarrow} H^{*}\left(G L_{n, k}, g l_{n}^{(r)}\right) .
$$


We show in Lemma 6.1 that the image of $e_{r} \in H^{2 p^{r-1}}\left(G L_{m, k}, g l_{m}^{(r)}\right)$ under the above homomorphism coincides with $e_{r} \in H^{2 p^{r-1}}\left(G L_{n, k}, g l_{n}^{(r)}\right)$.

Remark 1.2.2 Starting with cohomology classes $e_{i} \in H^{2 p^{i-1}}\left(G L_{n, k}, g l_{n}^{(i)}\right)$ and using the Frobenius morphism

$$
F: G L_{n, k} \rightarrow G L_{n, k}
$$

we can construct a few more rational cohomology classes. Note that if we start with a rational $G L_{n, k}$-module $g l_{n}^{(i)}$ and endow it with a new structure of a rational $G L_{n, k}$-module using the homomorphism $F: G L_{n, k} \rightarrow G L_{n, k}$, then this new rational $G L_{n, k}$-module is naturally isomorphic to $g l_{n}^{(i+1)}$. Thus, pulling back cohomology classes via $F$ defines a natural homomorphism

$$
H^{*}\left(G L_{n, k}, g l_{n}^{(i)}\right) \rightarrow H^{*}\left(G L_{n, k}, g l_{n}^{(i+1)}\right)
$$

We shall use the notation $\xi^{(1)}$ for the image of $\xi \in H^{*}\left(G L_{n, k}, g l_{n}^{(i)}\right)$ in $H^{*}\left(G L_{n, k}, g l_{n}^{(i+1)}\right)$. Applying this procedure to the cohomology classes $e_{i} \in$ $H^{2 p^{i-1}}\left(G L_{n, k}, g l_{n}^{(i)}\right)$ we get new cohomology classes $e_{i}^{(j)} \in H^{2 p^{i-1}}\left(G L_{n, k}, g l_{n}^{(i+j)}\right)$. Note that the restriction of $e_{i}^{(j)}$ to $\left(G L_{n, k}\right)_{(1)}$ is trivial if $j>0$.

In establishing that Theorem 1.2 implies Theorem 1.1, we start with the case of infinitesimal group schemes (i.e. connected finite group schemes). Let $G=\operatorname{Spec} A$ be an infinitesimal group scheme, let $\epsilon: A \rightarrow k$ be the augmentation defined by the unit of $G$ and let $\mathfrak{M}$ be the kernel of $\epsilon$. By definition, the ideal $\mathfrak{M}$ is nilpotent and hence there exists $N \geq 0$ such that $x^{p^{N}}=0$ for all $x \in \mathfrak{M}$. The minimal $N$ with this property is called the height of the infinitesimal group scheme $G$ and is denoted $h t(G)$. The height may be characterized also in slightly different terms as one sees from the following elementary Lemma, the proof of which we leave as an (easy) exercise to the reader.

Lemma 1.3. Let $G$ be an infinitesimal group scheme over $k$ and let $r \geq 0$ be an integer. The following conditions are equivalent

(1) $r \geq h t(G)$

(2) There exists a closed embeding $G \hookrightarrow\left(G L_{n, k}\right)_{(r)}$

(3) For any closed embeding $G \hookrightarrow G L_{n, k}$ the image of $G$ is contained in $\left(G L_{n, k}\right)_{(r)}$.

Assume that $G$ is an infinitesimal group scheme of height $r$. Choose an embeding $G \hookrightarrow\left(G L_{n, k}\right)_{(r)}$. Since the action of $G$ on $\left(g l_{n}\right)^{(r)}$ is trivial we get, restricting $e_{i}^{(r-i)}$ to $G$ the cohomology classes

$\left.e_{i}^{(r-i)}\right|_{G} \in H^{2 p^{i-1}}\left(G, g l_{n}^{(r)}\right)=H^{2 p^{i-1}}(G, k) \otimes g l_{n}^{(r)}=H_{o m}\left(\left(g l_{n}^{(r)}\right)^{\#}, H^{2 p^{i-1}}(G, k)\right)$.

In other words $e_{i}^{(r-i)}$ defines a $k$-linear map

$$
\left.e_{i}^{(r-i)}\right|_{G}:\left(g l_{n}^{(r)}\right)^{\#} \rightarrow H^{2 p^{i-1}}(G, k)
$$


and hence a homomorphism of graded $k$-algebras

$$
S^{*}\left(\left(g l_{n}^{(r)}\right)^{\#}\left(2 p^{i-1}\right)\right) \rightarrow H^{*}(G, k)
$$

Here the index $2 p^{i-1}$ in the parentheses indicates the degree in which $\left(g l_{n}^{(r)}\right)^{\#}$ is placed. Taking the product of all these homomorphisms we get a homomorphism

$$
\bigotimes_{i=1}^{r} S^{*}\left(\left(g l_{n}^{(r)}\right)^{\#}\left(2 p^{i-1}\right)\right) \rightarrow H^{*}(G, k)
$$

Lemma 1.4. Denote by $\operatorname{Tr} \in g l_{n}^{\#}$ the trace homomorphism. All the $k$-linear maps

$$
\left.e_{i}^{(r-i)}\right|_{G}:\left(g l_{n}^{(r)}\right)^{\#}=\left(g l_{n}^{\#}\right)^{(r)} \rightarrow H^{2 p^{i-1}}(G, k)
$$

vanish on the one-dimensional subspace $k \cdot \operatorname{Tr}^{(r)}$ and hence factor via $\left(\mathrm{gl}_{n}^{\#} / k\right.$. $\operatorname{Tr})^{(r)}=\left((\operatorname{Ker} \operatorname{Tr})^{\#}\right)^{(r)}=\left(s l_{n}^{\#}\right)^{(r)}$.

Proof. This follows from the commutativity of the diagram

$$
\begin{aligned}
& H^{2 p^{i-1}}\left(G L_{n, k}, g l_{n}^{(r)}\right) \longrightarrow H^{2 p^{i-1}}\left(G, g l_{n}^{(r)}\right)=\operatorname{Hom}_{k}\left(\left(g l_{n}^{(r)}\right)^{\#}, H^{2 p^{i-1}}(G, k)\right) \\
& \downarrow\left(T r^{(r)}\right)_{*} \quad \downarrow \circ T r^{(r)} \\
& H^{2 p^{i-1}}\left(G L_{n, k}, k\right) \quad \longrightarrow \quad H^{2 p^{i-1}}(G, k)
\end{aligned}
$$

since $H^{*}\left(G L_{n, k}, k\right)=0$ for $*>0$.

For infinitesimal groups our Theorem 1.1 may be given the following more constructive form.

Theorem 1.5. Let $G \subset\left(G L_{n, k}\right)_{(r)}$ be an infinitesimal group scheme over $k$ of height $r$. Let further $C$ be a commutative $k$-algebra (considered as a trivial $G$-module) and let $M$ be a noetherian $C$-module, on which $G$ acts by $C$-linear transformations. Assume that $n \not \equiv 0 \bmod p$. In this case $H^{*}(G, M)$ is a noetherian module over the algebra $\bigotimes_{i=1}^{r} S^{*}\left(\left(g l_{n}^{(r)}\right)^{\#}\left(2 p^{i-1}\right)\right) \otimes C$. In particular $H^{*}(G, k)$ is a finite module over a finitely generated algebra $\bigotimes_{i=1}^{r} S^{*}\left(\left(g l_{n}^{(r)}\right)^{\#}\left(2 p^{i-1}\right)\right)$ and hence is finitely generated.

Remark 1.5.1. Using remark 1.2 .1 one sees easily that the restriction $n \not \equiv 0$ $\bmod p$ is superfluous. However, the present formulation is sufficient for our purposes.

Corollary 1.5.2. Let $G$ be an infinitesimal group scheme of height $r$ over $k$ and let $M$ be a finite dimensional rational $G$-module. Then $H^{\mathrm{ev}}(G, k)$ is a finitely generated $k$-algebra and $H^{*}(G, M)$ is a finite $H^{\mathrm{ev}}(G, k)$-module.

Proof. Choose an embeding $G \hookrightarrow\left(G L_{n, k}\right)_{(r)}$ with $n \not \equiv 0 \bmod p$. Applying now Theorem 1.5 with $C=k$ we conclude that $H^{*}(G, M)$ is a noetherian module over the finitely generated $k$-algebra $A=\otimes_{i=1}^{r} S^{*}\left(\left(\left(g l_{n}\right)^{(r)}\right)^{\#}\left(2 p^{i-1}\right)\right)$. Since the action of the latter algebra on $H^{*}(G, M)$ factors via $H^{\mathrm{ev}}(G, k)$ we conclude that $H^{*}(G, M)$ 
is a noetherian (and hence finite) module over the algebra $H^{\mathrm{ev}}(G, k)$ as well. Finally taking $M=k$ we see that the algebra $H^{\mathrm{ev}}(G, k)$ is a finite module over the finitely generated $k$-algebra $A$ and hence is also finitely generated.

In the course of the proof of the Theorem 1.5 we will use several times the following elementary Lemma which is the main tool in proving finite generation via the spectral sequences. All the spectral sequences appearing in the next Lemma are assumed to consist of $k$-modules, $k$-algebras,... where $k$ is a fixed commutative ring, all tensor products are taken over $k$.

Lemma 1.6. Let $E_{2}^{p, q} \Longrightarrow E_{\infty}^{p+q}$ be a first quadrant (graded commutative) ring spectral sequence. Let further $\tilde{E}_{2}^{p, q} \Longrightarrow \widetilde{E}_{\infty}^{p+q}$ be a first quadrant spectral sequence which is a module over $E$. Assume further that we are given graded commutative rings $A$ and $B$ concentrated in even degrees and graded ring homomorphisms $A \rightarrow$ $E_{\infty}$ and $B \rightarrow E_{2}^{*, 0}$. Using the natural graded ring homomorphisms $E_{2}^{*, 0} \rightarrow E_{\infty}^{*, 0} \hookrightarrow$ $E_{\infty}$ and $E_{\infty} \rightarrow E_{\infty}^{0, *} \hookrightarrow E_{2}^{0, *}$ we produce further graded ring homomorphisms $A \otimes$ $B \stackrel{f}{\rightarrow} E_{\infty}$ and $A \otimes B \stackrel{g}{\rightarrow} E_{2}^{*, *}$. Assume that $g$ makes $\tilde{E}_{2}^{*, *}$ into a noetherian module over $A \otimes B$. Then $f$ makes $\tilde{E}_{\infty}$ into a noetherian module over $A \otimes B$.

Proof. Denote by $\Lambda_{r}$ the bigraded subalgebra of permanent cycles in $E_{r}^{*, *}$ and by $M_{r}$ denote the bigraded $\Lambda_{r}$-submodule of permanent cycles in $\tilde{E}_{r}^{* * *}$. For each $r$ $d_{r}\left(E_{r}^{*, *}\right)$ is an ideal in $\Lambda_{r}$ and $\Lambda_{r+1}=\Lambda_{r} / d_{r}\left(E_{r}^{*, *}\right)$. In the same way $\tilde{d}_{r}\left(\tilde{E}_{r}^{*, *}\right)$ is a $\Lambda_{r}$-submodule in $M_{r}$ and $M_{r+1}=M_{r} / \tilde{d}_{r}\left(\tilde{E}_{r}^{*, *}\right)$. Our definitions imply immediately that $g(A \otimes B) \subset \Lambda_{2}$ and hence all $M_{r}$ may be considered as $A \otimes B$-modules via $g$. Since $\widetilde{E}_{2}^{*, *}$ is a noetherian $A \otimes B$-module we conclude that $M_{2}$ is also a noetherian $A \otimes B$-module and hence the sequence of epimorphisms $M_{2} \rightarrow M_{3} \rightarrow \ldots$ consists of isomorphisms starting with a certain stage $r$. This implies that $\widetilde{d}_{i}=0$ for $i \geq r$ and hence $M_{r} \stackrel{\sim}{\longrightarrow} \widetilde{E}_{\infty}^{*, *}$. Thus we conclude that $\tilde{E}_{\infty}^{*, *}$ is a noetherian $A \otimes B$-module. The $A \otimes B$-module structure on $\tilde{E}_{\infty}^{*, *}$ is defined via the homomorphism $A \otimes B \rightarrow$ $\Lambda_{2} \rightarrow E_{\infty}^{*, *}$. Consider now the graded ring homomorphism $f: A \otimes B \rightarrow E_{\infty}$. Note that $f\left(B^{j}\right) \subset F^{j}\left(E_{\infty}^{j}\right)$ and hence if we define filtration on $A \otimes B$ via the formula

$$
F^{j}(A \otimes B)=A \otimes \bigoplus_{i \geq j} B^{i}
$$

then $f$ becomes a homomorphism of graded filtered rings. The associated bigraded ring for the filtered ring $A \otimes B$ may be identified canonically with $A \otimes B$ and the homomorphism

$$
g r(f): A \otimes B=\operatorname{gr}(A \otimes B) \rightarrow g r\left(E_{\infty}\right)=E_{\infty}^{*, *}
$$

coincides with the composition $A \otimes B \rightarrow \Lambda_{2} \rightarrow E_{\infty}^{*, *}$. Thus $\tilde{E}_{\infty}^{*, *}=\operatorname{gr}\left(\widetilde{E}_{\infty}\right)$ is a noetherian module over $\operatorname{gr}(A \otimes B)$ and hence $\widetilde{E}_{\infty}$ is a noetherian module over $A \otimes B$ (via $f$ ) - see [E] Proposition 2.1. 
Proof of the Theorem 1.5 (assuming Theorem 1.2). We start the proof of the Theorem 1.5 with a special case $r=1$. This case was already settled in [FP1], but since we need details of the computations we remind the proof. For any infinitesimal group scheme $G$ of height one the Hopf algebra $k[G]^{\#}$ is canonically isomorphic to the universal restricted envelopping algebra of the corresponding restricted Lie algebra $g$. Hence rational cohomology of $G$ coincides with cohomology of the restricted Lie algebra $g$ and hence for any rational $G$-module $M$ we have the May spectral sequence (see [M,FP2])

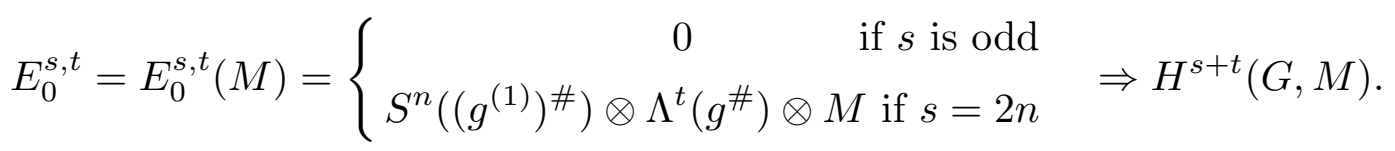

The differential $d_{0}$ of this spectral sequence coincides with the Koszul differential of the complex $\Lambda^{*}\left(g^{\#}\right) \otimes M$, whereas $d_{1}=0$ by dimension considerations. Thus

$$
E_{2}^{s, t}(M)=E_{1}^{s, t}(M)=\left\{\begin{array}{cl}
0 & \text { if } s \text { is odd } \\
S^{n}\left(\left(g^{(1)}\right)^{\#}\right) \otimes H^{t}(g, M) & \text { if } s=2 n
\end{array}\right.
$$

In the present situation, $E(C)$ is a ring spectral sequence and $E(M)$ is a module over $E(C)$. Furthermore $E_{2}^{2,0}(C)=E_{0}^{2,0}(C)=\left(g^{(1)}\right)^{\#} \otimes C$. Taking now $B=S^{*}\left(\left(g^{(1)}\right)^{\#}(2)\right)$ and $A=C$ (with trivial grading) in Lemma 1.6, we conclude immediately that $H^{*}(G, M)$ is a noetherian module over $S^{*}\left(\left(g^{(1)}\right)^{\#}(2)\right) \otimes C$. To finish the proof of the Theorem 1.5 for groups of height one, it suffices now to show that the homomorphism $\left(g l_{n}^{(1)}\right)^{\#} \rightarrow H^{2}(G, k)$ appearing in the formulation of Theorem 1.5 coincides (up to a non zero scalar factor) with the composition $\left(g l_{n}^{(1)}\right)^{\#} \rightarrow\left(g^{(1)}\right)^{\#} \rightarrow H^{2}(G, k)$ where the first arrow is obtained by duality from the map of Lie algebras $g \hookrightarrow g l_{n}$, corresponding to the closed embeding $G \hookrightarrow G L_{n, k}$ and the second arrow is the edge homomorphism in the May spectral sequence. In view of the naturality of the May spectral sequence it suffices to check the last statement in the special case $G=\left(G L_{n, k}\right)_{(1)}$. For future use, we prove the following more general result.

Lemma 1.7. Assume that $n \not \equiv 0 \bmod p$. Let $\left(g l_{n}^{(r)}\right)^{\#} \rightarrow H^{2 p^{r-1}}\left(\left(G L_{n, k}\right)_{(1)}, k\right)$ be a non zero $G L_{n, k}$-equivariant homomorphism vanishing on $\operatorname{Tr}^{(r)} \in\left(g l_{n}^{(r)}\right)^{\#}$. Then this homomorphism coincides (up to a non-zero scalar factor) with the composition

$$
\left(g l_{n}^{(r)}\right)^{\#} \rightarrow S^{p^{r-1}}\left(\left(g l_{n}^{(1)}\right)^{\#}\right) \rightarrow H^{2 p^{r-1}}\left(\left(G L_{n, k}\right)_{(1)}, k\right)
$$

where the first arrow is raising to the power $p^{r-1}$ and the second arrow is the edge homomorphism in the May spectral sequence.

Proof. From the May spectral sequence we extract the following exact sequence of rational $G L_{n, k}$-modules

$$
0 \rightarrow H^{1}\left(\left(G L_{n, k}\right)_{(1)}, k\right) \rightarrow H^{1}\left(g l_{n}, k\right) \stackrel{d_{2}}{\longrightarrow}\left(g l_{n}^{(1)}\right)^{\#} \rightarrow H^{2}\left(\left(G L_{n, k}\right)_{(1)}, k\right)
$$


An easy computation shows that $H^{1}\left(\left(G L_{n, k}\right)_{(1)}, k\right)=0$ whereas $H^{1}\left(g l_{n}, k\right)=k$. Since the only $G L_{n}$-invariant element in $\left(g l_{n}^{(1)}\right)^{\#}=\left(g l_{n}^{\#}\right)^{(1)}$ is $\operatorname{Tr}^{(1)}$ we conclude that the edge homomorphism $\left(g l_{n}^{\#}\right)^{(1)} \rightarrow H^{2}\left(\left(G L_{n, k}\right)_{(1)}, k\right)$ vanishes on $\operatorname{Tr}^{(1)}$ and hence factors via $\left(s l_{n}^{\#}\right)^{(1)}$. Using the multiplicative properties of the spectral sequence, we conclude next that the edge homomorphism $S^{p^{r-1}}\left(\left(g l_{n}^{\#}\right)^{(1)}\right) \rightarrow$ $H^{2 p^{r-1}}\left(\left(G L_{n, k}\right)_{(1)}, k\right)$ factors via $S^{p^{r-1}}\left(\left(s l_{n}^{\#}\right)^{(1)}\right)$. The assumption $n \not \equiv 0 \bmod p$ implies that $s l_{n}^{\#}=s l_{n}$ is an irreducible $G L_{n, k}$-module of high weight $\epsilon_{n}-\epsilon_{1}$ (see section 3 for conventions and notations pertaining to the representation theory of $\left.G L_{n}\right)$. Thus $\left(s l_{n}^{\#}\right)^{(r)}=s l_{n}^{(r)}$ is an irreducible $G L_{n}$-module of high weight $p^{r}\left(\epsilon_{n}-\epsilon_{1}\right)$. Immediate computation shows that the only term of total degree $2 p^{r-1}$ in the May spectral sequence in which this weight appears is $E_{2}^{2 p^{r-1}, 0}=S^{p^{r-1}}\left(\left(g l_{n}^{(1)}\right)^{\#}\right)$ and moreover it appears with multiplicity one. Thus there exists a unique (up to a scalar factor) homomorphism $\left(s l_{n}^{\#}\right)^{(r)} \rightarrow H^{2 p^{r-1}}\left(\left(G L_{n, k}\right)_{(1)}, k\right)$ and it coincides with the composition

$$
\left(s l_{n}^{\#}\right)^{(r)} \rightarrow S^{p^{r-1}}\left(\left(s l_{n}^{\#}\right)^{(1)}\right) \rightarrow H^{2 p^{r-1}}\left(\left(G L_{n, k}\right)_{(1)}, k\right) .
$$

Corollary 1.8. Let $G \subset\left(G L_{n, k}\right)_{(1)}$ be an infinitesimal group scheme of height 1 . let further $C$ be a commutative $k$-algebra (considered as a trivial $G$-module) and let $M$ be a noetherian $C$-module, on which $G$ acts by $C$-linear transformations. Assume that $n \not \equiv 0 \bmod p$. In this case for any $r \geq 1 \quad H^{*}(G, M)$ is a noetherian module over $S^{*}\left(\left(g l_{n}^{(r)}\right)^{\#}\left(2 p^{r-1}\right)\right) \otimes C$ (here $S^{*}\left(\left(g l_{n}^{(r)}\right)^{\#}\left(2 p^{r-1}\right)\right)$ is mapped to $H^{*}(G, k)$ using $\left.\left.e_{r}\right|_{G}\right)$.

Proof. According to what was proved above, $H^{*}(G, M)$ is a noetherian module over $S^{*}\left(\left(g l_{n}^{(1)}\right)^{\#}(2)\right) \otimes C$. Thus it suffices to show that the $k$-algebra homomorphism

$$
S^{*}\left(\left(g l_{n}^{(r)}\right)^{\#}\left(2 p^{r-1}\right)\right) \rightarrow H^{*}(G, k)
$$

may be factored via $S^{*}\left(\left(g l_{n}^{(1)}\right)^{\#}(2)\right)$ and that $S^{*}\left(\left(g l_{n}^{(1)}\right)^{\#}(2)\right)$ is a finite module over the (finitely generated) $k$-algebra $S^{*}\left(\left(g l_{n}^{(r)}\right)^{\#}\left(2 p^{r-1}\right)\right)$. As always it suffices to consider the special case $G=\left(G L_{n, k}\right)_{(1)}$. In this case the statement follows from the nontriviality of the restriction of $e_{r}$ to $\left(G L_{n, k}\right)_{(1)}$ and Lemmas 1.4 and 1.7.

Now we are prepared to settle the general case of Theorem 1.5. Let $G^{\prime} \subset$ $\left(G L_{n, k}\right)_{(r-1)}$ be the image of $G \subset\left(G L_{n, k}\right)_{(r)}$ under the Frobenius endomorphism $F: G L_{n, k} \rightarrow G L_{n, k}$ and let $H=G \cap\left(G L_{n, k}\right)_{(1)}$ be the kernel of the induced (surjective) homomorphism $F: G \rightarrow G^{\prime}$. Thus we have an extension of infinitesimal group schemes

$$
1 \rightarrow H \rightarrow G \rightarrow G^{\prime} \rightarrow 1
$$

and hence the associated Lindon-Hochschild-Serre spectral sequence (see $[J]$ )

$$
E_{2}^{s, t}(M)=H^{s}\left(G^{\prime}, H^{t}(H, M)\right) \Rightarrow H^{s+t}(G, M) .
$$

Set $A=S^{*}\left(\left(g l_{n}^{(r)}\right)^{\#}\left(2 p^{r-1}\right)\right) \otimes C, B=\bigotimes_{i=1}^{r-1} S^{*}\left(\left(g l_{n}^{(r-1)}\right)^{\#}\left(2 p^{i-1}\right)\right)$ and consider the standard maps (defined by $\left.e_{i}^{(r-1-i)}\right|_{G^{\prime}}$ and $\left.e_{r}\right|_{G}$ )

$$
B \rightarrow H^{*}\left(G^{\prime}, k\right)=E_{2}^{*, 0}(k), \quad A \rightarrow H^{*}(G, C) .
$$


Corollary 1.8 shows that $H^{*}(H, M)$ is a noetherian $A$-module. Applying now the induction hypothesis to the group $G^{\prime}$ of height $r-1$, the $k$-algebra $A$, and the noetherian $A$-module $H^{*}(H, M)$ on which $G^{\prime}$ acts by $A$-linear transformations, we conclude that $E_{2}^{*, *}(M)$ is a noetherian $A \otimes B$-module and hence $H^{*}(G, M)$ is a noetherian $A \otimes B$-module according to Lemma 1.6. Finally we remark that our definitions imply that for any $i=1, \ldots, r-1$ the following diagram commutes

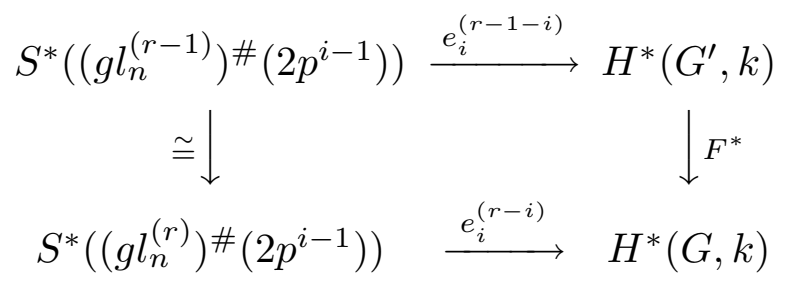

and hence the algebra $B \otimes A$ and the homomorphism $B \otimes A \rightarrow H^{*}(G, C)$ coincide with the corresponding data from the formulation of the Theorem 1.5.

To finish the proof of Theorem 1.1 we need two more Lemmas. The first one is the version of the Main Lemma of Evens [E].

Lemma 1.9. Let $G_{0}$ be a finite group scheme over $k$ and let $\pi$ be a finite group of order $n$ acting on $G_{0}$ by group scheme automorphisms. Consider the semidirect product $\pi \ltimes G_{0}=G$ and the natural embeding $G_{0} \hookrightarrow G$. Assume that $\xi \in H^{2 l}\left(G_{0}, k\right)$ is invariant under the action of $\pi$. Then there exists $\eta \in H^{2 \ln }(G, k)$ such that $\left.\eta\right|_{G_{0}}=\xi^{n}$.

Proof. Let $\pi$ act on $G_{0}^{\times \pi}$ permuting the factors : $\beta \cdot\left\{g_{\alpha}\right\}_{\alpha \in \pi}=\left\{g_{\beta^{-1} \alpha}\right\}_{\alpha \in \pi}$ and let $G_{0} \hookrightarrow G_{0}^{\times \pi}$ be the closed embeding given by the formula $g \mapsto\left\{\alpha^{-1} g\right\}_{\alpha \in \pi}$. This group homomorphism is compatible with the action of $\pi$ and hence defines a homomorphism on semidirect products

$$
G=\pi \ltimes G_{0} \hookrightarrow \pi \ltimes G_{0}^{\times \pi}=G_{0} \curlywedge \pi .
$$

Let $I^{\bullet}$ be an injective resolution of the $k\left[G_{0}\right]$-comodule $k$. Introduce some linear ordering on the set $\pi$ and consider the complex $\left(I^{\bullet}\right)^{\otimes \pi}$. This complex is an injective resolution of $k$ considered as a (trivial) comodule for $k\left[G_{0}^{\times \pi}\right]=k\left[G_{0}\right]^{\otimes \pi}$. Furthermore one can define the action of $\pi$ on this complex setting

$$
\beta \cdot\left(\otimes_{\alpha \in \pi} x_{\alpha}\right)=\epsilon\left(\beta, \operatorname{deg} x_{\alpha}\right) \cdot\left(\otimes_{\alpha \in \pi} x_{\beta^{-1} \alpha}\right) .
$$

Here $\epsilon$ is an appropriate sign depending on the chosen ordering of $\pi, \beta$ and the degrees of $x_{\alpha}$-see [E] page 231. The choice of $\epsilon$ is dictated by the necessity for this action to commute with the differential. One essential point is that $\epsilon=+1$ if degrees of all $x_{\alpha}$ are even. The actions of $k\left[G_{0}^{\times \pi}\right]$ and of $\pi$ on $\left(I^{\bullet}\right)^{\otimes \pi}$ are compatible and give rise to the action of the coalgebra $k\left[G_{0} 2 \pi\right]$ on $\left(I^{\bullet}\right)^{\otimes \pi}$. Since $\left(I^{\bullet}\right)^{\otimes \pi}$ is an acyclic $k\left[G_{0}<\pi\right]$-comodule complex over $k$ we have natural homomorphisms

$$
H^{*}\left(\left(\left[\left(I^{\bullet}\right)^{G_{0}}\right]^{\otimes \pi}\right)^{\pi}\right)=H^{*}\left(\left(\left(I^{\bullet}\right)^{\otimes \pi}\right)^{G_{0} 2 \pi}\right) \rightarrow H^{*}\left(G_{0} 2 \pi, k\right) \rightarrow H^{*}(G, k) .
$$

Let $x \in\left(I^{2 l}\right)^{G_{0}}$ be a representing cocycle for $\xi$. Then $x \otimes \ldots \otimes x \in\left(\left(\left(I^{\bullet}\right)^{G_{0}}\right)^{\otimes \pi}\right)^{\pi}$ is a cocycle and hence defines a cohomology class $\eta \in H^{2 l n}(G, k)$. A straightforward verification shows that $\left.\eta\right|_{G_{0}}=\xi^{n}$.

We leave the proof of the following elementary fact as an easy exercise to the reader 
Lemma 1.10. Let $A$ be a finitely generated commutative $k$-algebra and let $\pi$ be a finite group acting on $A$ by $k$-algebra automorphisms. Then $A$ is a finite module over the subalgebra of invariants $A^{\pi}$ and the algebra $A^{\pi}$ is finitely generated.

Proof of Theorem 1.1. Note that if $k^{\prime}$ is any extension of $k$ then for any affine group scheme $G / k$ and any rational $G$-module $M$ we have the following relation - see [J]

$$
H^{*}\left(G_{k^{\prime}}, M_{k^{\prime}}\right)=H^{*}(G, M) \otimes_{k} k^{\prime} .
$$

This formula shows that the validity of Theorem 1.1 for $G_{k^{\prime}}$ and $M_{k^{\prime}}$ implies its validity for $G$ and $M$ and enables us to replace $k$ by an appropriate extension $k^{\prime} / k$. Passing to an appropriate finite extension of $k$ we may assume that all points of the group scheme $G$ are $k$-rational. In this case $G$ is a semidirect product of its connected component $G_{0}$ and a finite group $\pi=G(k)$ of its $k$-points. Consider the Lyndon-Hochschild-Serre spectral sequences

$$
\begin{aligned}
E_{2}^{s t}(k) & =H^{s}\left(\pi, H^{t}\left(G_{0}, k\right)\right) \Rightarrow H^{s+t}(G, k) \\
E_{2}^{s t}(M) & =H^{s}\left(\pi, H^{t}\left(G_{0}, M\right)\right) \Rightarrow H^{s+t}(G, M) .
\end{aligned}
$$

According to Corollary 1.5.2 $H^{*}\left(G_{0}, M\right)$ is a finite module over the finitely generated commutative $k$-algebra $H^{\mathrm{ev}}\left(G_{0}, k\right)$. Furthermore Lemma 1.10 implies that $H^{*}\left(G_{0}, M\right)$ is a finite module even over the subalgebra $H^{\mathrm{ev}}\left(G_{0}, k\right)^{\pi}$. Choose a finite system of generators $\xi_{i} \in H^{2 l_{i}}\left(G_{0}, k\right)^{\pi}$ of the latter algebra and denote the order of $\pi$ by $n$. According to Lemma 1.9, there exist cohomology classes $\eta_{i} \in H^{2 l_{i} n}(G, k)$ such that $\left.\eta_{i}\right|_{G_{0}}=\xi_{i}^{n}$. Let $A$ be the subalgebra of $H^{*}(G, k)$ generated by $\eta_{i}$ and let $B$ be the algebra $H^{\mathrm{ev}}(\pi, k)$. The algebra $B$ is finitely generated according to the theorem of Venkov-Evens ([V,E]). Furthermore the action of $\pi$ on $A$ is trivial and $H^{*}\left(G_{0}, M\right)$ is a finite $A$-module according to our construction. A theorem of Evens [E] now shows that $H^{*}\left(\pi, H^{*}\left(G_{0}, M\right)\right)$ is a finite $B \otimes A$-module. Using once again Lemma 1.6, we conclude that $H^{*}(G, M)$ is a finite $B \otimes A$-module. Taking $M=k$ we see that $H^{*}(G, k)$ is a finite module over a finitely generated algebra $B \otimes A$ and hence is also finitely generated.

\section{$\S 2$. The Category $\mathcal{P}$ of polynomial functors of Finite Degree.}

In this section, we establish basic properties of the category $\mathcal{P}$ (of polynomial functors of finite degree) in which we shall make computations of Ext-groups in order to prove Theorem 1.2. The two key results are Theorem 2.10 which constructs convenient projective (and injective) functors and Theorem 2.13 which provides a very useful vanishing theorem.

Let $k$ be a field. Denote by $\mathcal{V}$ the category of finite dimensional vector spaces over $k$ and $k$-linear maps. For any $V, W \in \mathcal{V}$ set $\operatorname{Hom}_{P o l}(V, W)=S^{*}\left(V^{\#}\right) \otimes W$, where $V^{\#}$ is the $k$-linear dual of $V$ and $S^{*}\left(V^{\#}\right)$ is the symmetric algebra on $V^{\#}$. The elements of $\operatorname{Hom}_{P o l}(V, W)$ are called polynomial maps from $V$ to $W$. A polynomial map is said to be homogenous of degree $d$ if it belongs to $S^{d}\left(V^{\#}\right) \otimes W$.

Observe that every polynomial map $p: V \rightarrow W$ determines a set map from $V$ to $W$ (which we will usually denote by the same letter $p$ ) and is determined by this set map provided that the field $k$ is infinite. Thus over infinite fields polynomial maps 
may be identified with set maps $p: V \rightarrow W$ having the property that (with respect to some choice of bases for $V$ and $W$ ) the coordinates of $p(v) \in W$ are polynomial functions in coordinates of $v \in V$.

Quite often we will be identifying vector space $V$ over $k$ with the corresponding affine scheme Spec $S^{*}\left(V^{\#}\right)$. It's clear that with this identification we have the following formula:

$$
\operatorname{Hom}_{P o l}(V, W)=\operatorname{Hom}_{S c h / k}(V, W) .
$$

The last formula enables us in particular to define a composition map

$$
\operatorname{Hom}_{P o l}(V, W) \times H_{\text {omol }}(U, V) \rightarrow H_{\text {omol }}(U, W) .
$$

Note also that we can extend scalars in polynomial maps, so that whenever $p: V \rightarrow$ $W$ is a polynomial map and $k^{\prime} / k$ is a field extension we may consider a polynomial map $p_{k^{\prime}}: V_{k^{\prime}} \rightarrow W_{k^{\prime}}$ (and hence also the corresponding set map $V_{k^{\prime}} \rightarrow W_{k^{\prime}}$ ). Note finally that $\operatorname{Hom}_{k}(V, W)=V^{\#} \otimes W \subset \operatorname{Hom}_{P o l}(V, W)$, i.e. $k$-linear maps may be identified with homogenous polynomial maps of degree one. In the same way each bilinear map $U \times V \rightarrow W$ defines a homogenous polynomial map of degree 2 .

Definition 2.1. A polynomial functor $T: \mathcal{V} \rightarrow \mathcal{V}$ is a pair of functions, the first of which assigns to each $V \in \mathcal{V}$ a new vector space $T(V) \in \mathcal{V}$ and the second assignes a polynomial map $T_{V, W} \in H_{\text {om }}\left(H_{\text {or }}(V, W), H_{k}(T(V), T(W))\right)$ to each pair $V, W \in \mathcal{V}$. These two functions should satisfy the usual conditions from the definition of a functor, which now take the form:

(2.1.1) For any vector space $V \in \mathcal{V}$ we have: $T_{V, V}\left(1_{V}\right)=1_{T(V)}$.

(2.1.2) For any $U, V, W \in \mathcal{V}$ the following diagram of polynomial maps commute

$$
\begin{aligned}
& \operatorname{Hom}_{k}(V, W) \times \operatorname{Hom}_{k}(U, V) \quad \longrightarrow \quad H_{k}(U, W) \\
& T_{V, W} \times T_{U, V} \downarrow \downarrow \downarrow T_{U, W} \\
& H \operatorname{mom}_{k}(T(V), T(W)) \times \operatorname{Hom}_{k}(T(U), T(V)) \longrightarrow \operatorname{Hom}_{k}(T(U), T(W)) \text {. }
\end{aligned}
$$

Note that over an infinite field $k$ one can identify polynomial functors with usual functors $T: \mathcal{V} \rightarrow \mathcal{V}$ which have the property that for any $V, W \in \mathcal{V}$ the natural map of $k$-vector spaces $T: \operatorname{Hom}_{k}(V, W) \rightarrow H_{o m}(T(V), T(W))$ is polynomial. Thus the above definition is really necessary only dealing with finite fields.

Let $G / k$ be an affine group scheme and let $V$ be a finite dimensional representation of $G$. Thus we are given a homomorphism of $k$-groups $G \rightarrow G L(V)$. Assume further that $T$ is a polynomial functor. By definition, $T$ defines a morphism of schemes over $k \quad T_{V, V}: \operatorname{End}_{k}(V) \rightarrow \operatorname{End}_{k}(T(V))$. Moreover one checks easily that this morphism takes the open subscheme $G L(V) \subset \operatorname{End}_{k}(V)$ to $G L(T(V)) \subset$ $\operatorname{End}_{k}(T(V))$ and defines a homomorphism of group schemes over $k \quad T: G L(V) \rightarrow$ $G L(T(V))$. Composing this homomorphism with the original homomorphism $G \rightarrow$ $G L(V)$ we see that we have a natural representation of $G$ in $T(V)$ as well.

The above remark explains the role of polynomial functors in construction of representations of affine group schemes. 
Lemma 2.2. Let $T: \mathcal{V} \rightarrow \mathcal{V}$ be a polynomial functor and let $n \geq 0$ be an integer then the following conditions are equivalent

(1) For any $V \in \mathcal{V}$, any field extension $k^{\prime} / k$, and any $\lambda \in k^{\prime *}$, the $k^{\prime}$-linear map $T_{k^{\prime}}\left(\lambda \cdot 1_{V_{k^{\prime}}}\right) \in \operatorname{End}_{k^{\prime}}\left(T(V)_{k^{\prime}}\right)$ coincides with $\lambda^{n} \cdot 1_{T(V)_{k^{\prime}}}$

(2) For any $V \in \mathcal{V}, n$ is the only weight of the representation of the algebraic group $\mathbf{G}_{\mathbf{m}}$ in $T(V)$ obtained by applying $T$ to the evident representation of $\mathbf{G}_{\mathbf{m}}$ in $V$.

(3) For any $V, W \in \mathcal{V}$, the polynomial map $T_{V, W}: \operatorname{Hom}_{k}(V, W) \rightarrow$ $\rightarrow H_{k}(T(V), T(W))$ is homogenous of degree $n$.

A polynomial functor satisfying one of these equivalent conditions is said to be homogenous of degree $n$.

Proof. The equivalence of (1) and (2) is straightforward. Furthermore it is clear that (3) implies (1). Assume now that (1) is satisfied. Let $k^{\prime} / k$ be any infinite field extension of $k$. Note that the polynomial map $k^{\prime} \rightarrow \operatorname{End}_{k^{\prime}}\left(V_{k^{\prime}}\right): \quad \lambda \mapsto T_{k^{\prime}}(\lambda$. $\left.1_{V_{k^{\prime}}}\right)-\lambda^{n} \cdot 1_{T(V)_{k^{\prime}}}$ takes zero value for all $\lambda \neq 0$ and hence takes zero value for $\lambda=0$ as well. Consider now the polynomial map $T_{V, W}^{\prime}=\left(T_{V, W}\right)_{k^{\prime}}: H_{o m} m_{k^{\prime}}\left(V_{k^{\prime}}, W_{k^{\prime}}\right) \rightarrow$ $H_{o^{\prime} m_{k^{\prime}}}\left(T(V)_{k^{\prime}}, T(W)_{k^{\prime}}\right)$. For any $f \in H_{o^{\prime}}\left(V_{k^{\prime}}, W_{k^{\prime}}\right)$ and any $\lambda \in k^{\prime}$ we have $T_{V, W}^{\prime}(\lambda \cdot f)=T_{V, W}^{\prime}\left(\left(\lambda \cdot 1_{W_{k^{\prime}}}\right) \circ f\right)=T_{W, W}^{\prime}\left(\lambda \cdot 1_{W_{k^{\prime}}}\right) \circ T_{V, W}^{\prime}(f)=\lambda^{n} \cdot T_{V, W}^{\prime}(f)$. This shows that the polynomial map $T_{V, W}^{\prime}$ and hence also $T_{V, W}$ is homogenous of degree $n$.

Here is a list of standard examples of homogenous polynomial functors. In each case we indicate only the action of the functor on vector spaces (the corresponding polynomial maps are more or less self evident).

(i.) $\otimes^{d}: \mathcal{V} \rightarrow \mathcal{V}$ is the polynomial functor sending a vector space $V$ to its $d$-th tensor power $V^{\otimes d}$.

(ii.) $\Lambda^{d}: \mathcal{V} \rightarrow \mathcal{V}$ is the polynomial functor sending a vector space $V$ to its $d$-th exterior power $\Lambda^{d}(V)$ defined as an appropriate quotient of $V^{\otimes d}$.

(iii.) $S^{d}: \mathcal{V} \rightarrow \mathcal{V}$ is the polynomial functor sending a vector space $V$ to its $d$-th symmetric power $S^{d}(V)=\left(V^{\otimes d}\right)_{\Sigma_{d}}$ where $\Sigma_{d}$ is the symmetric group on $d$ letters.

(iv.) $\Gamma^{d}: \mathcal{V} \rightarrow \mathcal{V}$ is the polynomial functor sending a vector space $V$ to its $d$-th divided power $\Gamma^{d}(V)=\left(V^{\otimes d}\right)^{\Sigma_{d}}$.

(v.) Assume that $k$ is a field of positive characteristic $p>0$. The Frobenius twist $V \mapsto V^{(1)}$, discussed in the previous section is a homogenous polynomial functor of degree $p$.

Observe that (for $\mathrm{k}$ infinite) the polynomial functor $(-)^{(1)}$ is an equivalence of categories, but its inverse $(-)^{(-1)}$ is not a polynomial functor.

Let $S$ and $T$ be polynomial functors. A natural transformation (or functor homomorphism) $f: S \rightarrow T$ is a collection of $k$-linear maps $f_{V}: S(V) \rightarrow T(V)$ given for all $V \in \mathcal{V}$ and satisfying the following property. For any $V, W \in \mathcal{V}$ the 
following diagram of polynomial maps commute

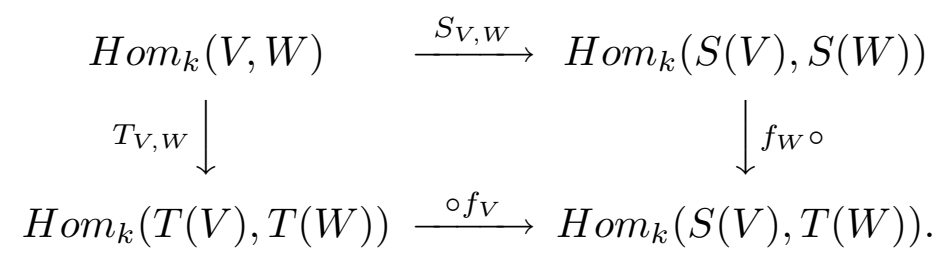

Having defined homomorphism of functors we may consider the category whose objects are polynomial functors and whose morphisms are functor homomorphisms. One checks easily that this category is abelian.

Let $T: \mathcal{V} \rightarrow \mathcal{V}$ be any polynomial functor and let $V \in \mathcal{V}$ be a vector space. Consider the representation of the algebraic group $\mathbf{G}_{\mathbf{m}}$ in $T(V)$, obtained by applying $T$ to the evident representation of $\mathbf{G}_{\mathbf{m}}$ in $V$. Since the group $\mathbf{G}_{\mathbf{m}}$ is diagonalisible we conclude that $T(V)$ is a direct sum of weight subspaces $T_{n}(V)$ (see $[\mathrm{J}]$ ). The weight subspace $T_{n}(V)$ has the following description:

$$
T_{n}(V)=\left\{x \in T(V): T\left(\lambda \cdot 1_{V_{k^{\prime}}}\right)(x \otimes 1)=\lambda^{n} \cdot(x \otimes 1), \forall \lambda \in k^{\prime} \quad \forall k^{\prime} / k\right\} .
$$

Using this description one checks easily that $T_{n}$ is a polynomial subfunctor of $T$ (called the $n$-th homogenous component of $T$ ). Finally since the action of $\mathbf{G}_{\mathbf{m}}$ in $T(V)$ is polynomial we conclude easily that $T_{n}(V)=0$ for $n<0$. We derive immediately from Lemma 2.2 the following assertion

Corollary 2.3. $\max \left(n: T_{n}(V) \neq 0\right)$ coincides with the degree of the polynomial $\operatorname{map} T: \operatorname{End}_{k}(V) \rightarrow \operatorname{End}_{k}(T(V))$

Definition 2.4. We will say that the polynomial functor $T$ is a functor of finite degree if the degrees of the polynomial maps $T: \operatorname{End}_{k}(V) \rightarrow \operatorname{End}_{k}(T(V))(V \in \mathcal{V})$ are bounded above. The maximum of these degrees is called the degree of $T$ and denoted $\operatorname{deg} T$.

The following Lemma is clear from the previous discussion.

Lemma 2.5. Any polynomial functor of finite degree $T$ is equal to the direct sum of it's homogenous components : $T=\bigoplus_{n=0}^{\operatorname{deg} T} T_{n}$.

The preceding argument easily implies the following splitting of the category $\mathcal{P}$. The other assertions of the following proposition are essentially self-evident.

Proposition 2.6. Let $\mathcal{P}$ denote the abelian category of polynomial functors of finite degree. Then

$$
\mathcal{P}=\bigoplus_{d \geq 0} \mathcal{P}_{d},
$$

where $\mathcal{P}_{d} \subset \mathcal{P}$ denotes the full subcategory of homogeneous polynomial functors of degree $d$.

Tensor product determines a biexact functor

$$
\otimes: \mathcal{P}_{d} \times \mathcal{P}_{e} \rightarrow \mathcal{P}_{d+e}
$$


Moreover, if we define the functor $T^{\#}$ by setting $T^{\#}(V)=T\left(V^{\#}\right)^{\#}$, then sending $T$ to $T^{\#}$ determines an equivalence of categories

$$
(-)^{\#}: \mathcal{P}^{o p} \rightarrow \mathcal{P}
$$

which preserves degree.

Classical linear algebra enables us to conclude that $\Lambda^{d}$ is self-dual (i.e., $\Lambda^{d \#}=$ $\Lambda^{d}$ ) and that $S^{d}, \Gamma^{d}$ are mutually dual (i.e., $S^{d \#}=\Gamma^{d}$ and $\Gamma^{d \#}=S^{d}$ ).

Note that to give a polynomial map $p: V \rightarrow W$ is the same as to give a $k$ linear homomorphism $\alpha_{p}: \bigoplus_{d=0}^{\infty} \Gamma^{d}(V) \rightarrow W$ which is zero on all but finitely many components. The relationship between $p$ and $\alpha_{p}$ is given by the formula $p(v)=\alpha_{p}\left(\sum_{d=0}^{\infty} v^{\otimes d}\right) \quad \forall v \in V$ (this expression makes sense since $\alpha_{p}$ is zero on all but finitely many components). Hence, a polynomial functor $T$ determines for any $V, W \in \mathcal{V}$ a natural $k$-linear map $\bigoplus_{d=0}^{\infty} \Gamma^{d}\left(\operatorname{Hom}_{k}(V, W)\right) \otimes T(V) \rightarrow T(W)$ (equal to zero on all but finitely many components).

Lemma 2.7. Let $T$ be a polynomial functor and let $V, W \in \mathcal{V}$ be two vector spaces. The following conditions are equivalent:

(1) For any infinite extension $k^{\prime} / k$ the natural map

$$
\bigoplus_{f \in H_{o m} m_{k^{\prime}}\left(V_{k^{\prime}}, W_{k^{\prime}}\right)} T(V)_{k^{\prime}} \stackrel{\sum T_{k^{\prime}}(f)}{\longrightarrow} T(W)_{k^{\prime}}
$$

is surjective.

(2) There exists an extension $k^{\prime} / k$ such that the above natural map is surjective.

(3) The natural map $\bigoplus_{d=0}^{\infty} \Gamma^{d}\left(\operatorname{Hom}_{k}(V, W)\right) \otimes T(V) \rightarrow T(W)$ is surjective.

Proof. It's clear that (1) implies (2). Furthermore (2) implies that the natural map $\bigoplus_{d=0}^{\infty} \Gamma^{d}\left(H_{o m}(V, W)\right) \otimes T(V) \rightarrow T(W)$ becomes surjective after tensoring with $k^{\prime}$ over $k$ and hence is surjective. Finally to show that (3) implies (1) we use the following Lemma

Lemma 2.7.1. Assume that the field $k$ is infinite. Then for any $V \in \mathcal{V}$ and any $N>0$, the $k$-vector space $\bigoplus_{d=0}^{N} \Gamma^{d}(V)$ is generated by elements of the form $\sum_{d=0}^{N} v^{\otimes d} \quad(v \in V)$

Proof. The dual vector space to $\bigoplus_{d=0}^{N} \Gamma^{d}(V)$ coincides with $\bigoplus_{d=0}^{N} S^{d}\left(V^{\#}\right)$. Choosing a basis for $V$ we identify $V$ with $k^{n}$ for an appropriate $n$. After this identification the vector space $\bigoplus_{d=0}^{N} S^{d}\left(V^{\#}\right)$ may be identified with the vector space of polynomials of degree $\leq N$ in $n$ variables. Now our statement takes the following form (in which it is evident) : if a polynomial (of degree $\leq N$ ) takes zero values in all rational points $v \in k^{n}$ then it is zero.

Definition 2.7.2. A polynomial functor $T$ is said to be $\mathbf{n}$-generated if the natural map $\bigoplus_{d=0}^{\infty} \Gamma^{d}\left(H_{o m}\left(k^{n}, V\right)\right) \otimes T\left(k^{n}\right) \rightarrow T(V)$ is surjective for all $V \in \mathcal{V}$. If $T$ is $n$-generated for some $n \geq 0$, then $T$ is said to be finitely generated. 
Lemma 2.8. Let $h: V \rightarrow W$ be a homogenous polynomial map of degree $d \geq 0$. Then for any $n>d$ and any $v_{1}, \ldots, v_{n} \in V$, the following equality holds:

$$
\begin{gathered}
h\left(v_{1}+\cdots+v_{n}\right)-\sum_{i} h\left(v_{1}+\cdots+\hat{v}_{i}+\cdots+v_{n}\right) \\
+\sum_{i_{1}<i_{2}} h\left(v_{1}+\cdots+\hat{v}_{i_{1}}+\cdots+\hat{v}_{i_{2}}+\cdots+v_{n}\right)-\cdots+(-1)^{n} h(0)=0
\end{gathered}
$$

Consequently, $h\left(v_{1}+\ldots+v_{n}\right)$ is equal to an integral linear combination of $h\left(v_{s_{1}}+\right.$ $\left.\ldots+v_{s_{j}}\right)$ with $j \leq d$.

Proof. We may assume that $W=k$ and $h$ is a product of linear forms: $h=h^{1} \cdot \ldots \cdot h^{d}$. The above expression is a linear combination of products of the form $h^{1}\left(v_{j_{1}}\right) \cdot \ldots$. $h^{d}\left(v_{j_{d}}\right)$. This product appears in $h\left(v_{1}+\cdots+\hat{v}_{i_{1}}+\cdots+\hat{v}_{i_{s}}+\cdots+v_{n}\right)$ with coefficient 1 if $\left\{i_{1}, \ldots, i_{s}\right\} \cap\left\{j_{1}, \ldots, j_{d}\right\}=\emptyset$ and with coefficient 0 otherwise. Denoting the cardinality of the set $\{1, \ldots, n\} \backslash\left\{j_{1}, \ldots, j_{d}\right\}$ by $m$ and noting that $m \geq n-d>0$, we conclude that the total coefficient with which $h^{1}\left(v_{j_{1}}\right) \cdot \ldots \cdot h^{d}\left(v_{j_{d}}\right)$ appears in the above expression is equal to $1-m+\left(\begin{array}{c}m \\ 2\end{array}\right)-\ldots+(-1)^{m}\left(\begin{array}{c}m \\ m\end{array}\right)=(1-1)^{m}=0$.

Proposition 2.9. A polynomial functor $T$ is finitely generated if and only if it is of finite degree. More precisely, if $T$ is n-generated, then deg $(T)$ is the degree of the polynomial map $T: \operatorname{End}_{k}\left(k^{n}\right) \rightarrow \operatorname{End}_{k}\left(T\left(k^{n}\right)\right)$; conversely, if $T$ has degree $d$, then $T$ is d-generated.

Proof. Assume that $T$ is $n$-generated and set $d$ equal to the degree of the polynomial map $T: \operatorname{End}_{k}\left(k^{n}\right) \rightarrow \operatorname{End}_{k}\left(T\left(k^{n}\right)\right)$. Corollary 2.3 shows that $T\left(k^{n}\right)=$ $\bigoplus_{j=0}^{d} T_{j}\left(k^{n}\right)$. Let now $V \in \mathcal{V}$ be any vector space. Choose an infinite extension $k^{\prime} / k$. Since for any $f \in H_{o m} k_{k^{\prime}}\left(k^{\prime n}, V_{k^{\prime}}\right)$ the $k^{\prime}$-linear map $T_{k^{\prime}}(f): T\left(k^{n}\right)_{k^{\prime}} \rightarrow$ $T(V)_{k^{\prime}}$ takes $\left(T_{j}\left(k^{n}\right)\right)_{k^{\prime}}$ to $T_{j}(V)_{k^{\prime}}$, we conclude that $T(V)_{k^{\prime}}=\bigoplus_{j=0}^{d} T_{j}(V)_{k^{\prime}}$ and hence $T(V)=\bigoplus_{j=0}^{d} T_{j}(V)$. Corollary 2.3 shows now that the degree of the polynomial map $T: \operatorname{End}_{k}(V) \rightarrow \operatorname{End}_{k}(T(V))$ is at most $d$.

To prove the converse, it suffices to consider a homogeneous polynomial functor $T$ of degree $d$ and prove the surjectivity of the natural homomorphism

$$
\bigoplus_{f: k^{d} \rightarrow k^{m}} T\left(k^{d}\right) \stackrel{\sum T(f)}{\longrightarrow} T\left(k^{m}\right)
$$

for all $m$. If $m \leq d$, choose an embeding $i: k^{m} \hookrightarrow k^{d}$ and a projection $p: k^{d} \rightarrow k^{m}$ such that $p \circ i=1_{k^{m}}$. Since $T(p) \circ T(i)=1_{T\left(k^{m}\right)}$, we conclude that the single map $T(p)$ is surjective.

If $m>d$, let $p_{i}: k^{m} \rightarrow k^{m}$ denote the projection onto the $i$-th coordinate: $p_{i}\left(x_{1}, \ldots, x_{m}\right)=\left(0, \ldots, x_{i}, \ldots, 0\right)$. Thus, $1_{k^{m}}=\sum_{i=1}^{m} p_{i}$. Lemma 2.8 implies that $1_{T\left(k^{m}\right)}$ is a linear combination of maps $T\left(p_{i_{1}}+\cdots+p_{i_{s}}\right)$ with $s \leq d$. Since each such $p_{i_{1}}+\cdots+p_{i_{s}}: k^{m} \rightarrow k^{m}$ factors through $k^{d}$, we conclude the asserted surjectivity.

As the theorem below shows, the abelian category $\mathcal{P}$ has enough projectives. Indeed, the very explicit functor (homogeneous of degree $d$ )

$$
\Gamma^{d, n}=\Gamma^{d} \circ \operatorname{Hom}_{k}\left(k^{n},-\right): \mathcal{V} \rightarrow \mathcal{V}
$$


serves as a projective generator of $\mathcal{P}_{d}$ provided that $n \geq d$. Of course, the dual

$$
S^{d, n}=\left(\Gamma^{d, n}\right)^{\#}
$$

is an injective object of $\mathcal{P}_{d}$, so that $\mathcal{P}$ also has enough injectives.

Theorem 2.10. For any homogeneous polynomial functor $T$ of degree $d$ and any $n>0$,

$$
\operatorname{Hom}_{\mathcal{P}_{d}}\left(\Gamma^{d, n}, T\right)=T\left(k^{n}\right) .
$$

Thus, $\Gamma^{d, n}$ is a projective object in $\mathcal{P}_{d}$.

Moreover, for any homogeneous polynomial functor $T$ the associated natural transformation (natural with respect to $T$ )

$$
\theta: T\left(k^{n}\right) \otimes \Gamma^{d, n} \rightarrow T
$$

is surjective if and only if $T$ is n-generated. Hence, $\Gamma^{d, n}$ is a projective generator of $\mathcal{P}_{d}$ provided that $n \geq d$.

Proof. For any homogenous polynomial functor $T$ of degree $d$ and any vector space $V \in \mathcal{V}$ we have a natural $k$-linear map $\theta_{V}: \Gamma^{d}\left(H_{o m}\left(k^{n}, V\right)\right) \otimes T\left(k^{n}\right) \rightarrow T(V)$. The homomorphism $\theta_{V}$ depends functorially on $V$. Thus we get a canonical natural transformation $\theta: T\left(k^{n}\right) \otimes \Gamma^{d, n} \rightarrow T$ determining a $k$-linear map

$$
T\left(k^{n}\right) \rightarrow H o m_{\mathcal{P}}\left(\Gamma^{d, n}, T\right) .
$$

On the other hand, to each homomorphism of functors $\alpha: \Gamma^{d, n} \rightarrow T$ we can associate an element $\alpha_{k^{n}}\left(1_{k^{n}} \otimes \ldots \otimes 1_{k^{n}}\right) \in T\left(k^{n}\right)$, thus defining a $k$-linear map $\operatorname{Hom}_{\mathcal{P}}\left(\Gamma^{d, n}, T\right) \rightarrow T\left(k^{n}\right)$. A straightforward verification shows that these two maps relating $\operatorname{Hom}_{\mathcal{P}}\left(\Gamma^{d, n}, T\right)$ and $T\left(k^{n}\right)$ are mutually inverse.

The last statement is evident from the definitions.

Corollary 2.11. The tensor product of any two projective objects of $\mathcal{P}$ is again projective

Proof. It suffices to establish the projectivity of the functor $\Gamma^{d, n} \otimes \Gamma^{e, m}$. The projectivity of this functor follows immediately from the formula $\Gamma^{d+e, n+m}=$ $\bigoplus_{i+j=d+e} \Gamma^{i, n} \otimes \Gamma^{j, m}$.

The functor $\Gamma^{d, n}$ splits canonically into direct sum

$$
\Gamma^{d, n}(V)=\Gamma^{d}\left(H_{o m}\left(k^{n}, V\right)\right)=\Gamma^{d}\left(V^{n}\right)=\bigoplus_{d_{1}+\ldots+d_{n}=d} \Gamma^{d_{1}}(V) \otimes \ldots \otimes \Gamma^{d_{n}}(V) .
$$

Quite often it's more convenient to work with summands of this decomposition.

Corollary 2.12. For any $T \in \mathcal{P}_{d}$, the rational representation of $\mathbf{G}_{\mathbf{m}}{ }^{\times n}$ on $T\left(k^{n}\right)$ provides a natural splitting of $T\left(k^{n}\right)$ as $\mathbf{G}_{\mathbf{m}}{ }^{\times n}$-eigenspaces

$$
T\left(k^{n}\right)=\bigoplus_{d_{1}+\cdots+d_{n}=d} T\left(k^{n}\right)^{d_{1}, \ldots, d_{n}}
$$


where the sum is indexed by non-negative integers $d_{1}, \ldots, d_{n}$ which sum to $d$. Moreover, the identification of $\operatorname{Hom}_{\mathcal{P}}\left(\Gamma^{d, n}, T\right)$ with $T\left(k^{n}\right)$ restricts to an identification

$$
\operatorname{Hom}_{\mathcal{P}}\left(\Gamma^{d_{1}} \otimes \cdots \otimes \Gamma^{d_{n}}, T\right)=T\left(k^{n}\right)^{d_{1}, \ldots, d_{n}} .
$$

Proof. The splitting is merely the statement of semi-simplicity of $\mathbf{G}_{\mathbf{m}}{ }^{\times n}$. More explicitly, $T\left(k^{n}\right)^{d_{1}, \ldots, d_{n}} \subset T\left(k^{n}\right)$ consists of those $x \in T\left(k^{n}\right)$ with the property that $T_{k^{\prime}}\left(\operatorname{diag}\left(\lambda_{1}, \ldots, \lambda_{n}\right)\right)$ sends $x$ to $\lambda_{1}^{d_{1}} \cdots \lambda_{n}^{d_{n}} \cdot x$ for any $\left(\lambda_{1}, \ldots, \lambda_{n}\right) \in \mathbf{G}_{\mathbf{m}}{ }^{\times n}\left(k^{\prime}\right)$ and any extension $k^{\prime} / k$. Using the explicit formula for the inclusion $\Gamma^{d_{1}} \otimes \ldots \otimes \Gamma^{d_{n}} \hookrightarrow \Gamma^{d, n}$ we readily verify the commutativity of the following diagram

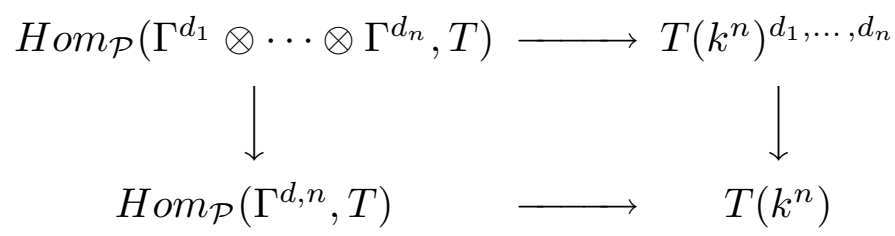

in which the lower horizontal map sends $\alpha: \Gamma^{d, n} \rightarrow T$ to $\alpha_{k^{n}}\left(1_{k^{n}} \otimes \cdots 1_{k^{n}}\right)$ as in the proof of Theorem 2.10 and the upper horizontal map sends $\beta: \Gamma^{d_{1}} \otimes \cdots \otimes \Gamma^{d_{n}} \rightarrow T$ to $\beta_{k^{n}}\left((\underbrace{e_{1} \otimes \ldots \otimes e_{1}}_{d_{1}}) \otimes \cdots \otimes(\underbrace{e_{n} \otimes \ldots \otimes e_{n}}_{d_{n}})\right)$

We conclude this section with a vanishing result which provides the key tool in our computation of Ext-groups leading to the proof of Theorem 1.1. Our theorem is merely a variant of a vanishing theorem of M. Jibladze and T. Pirashvili [J-P] (see also [F-L-S]).

Theorem 2.13. Let $T$ and $T^{\prime}$ be homogenous polynomial functors of positive degree and let $A \in \mathcal{P}$ be additive. Then $\operatorname{Ext}_{\mathcal{P}}^{*}\left(A, T \otimes T^{\prime}\right)=0$.

Proof. Let $e \in \operatorname{Ext}_{\mathcal{P}}^{k}\left(A, T \otimes T^{\prime}\right)$ be given by an extension of functors

$$
E: \quad 0 \rightarrow T \otimes T^{\prime} \rightarrow U^{1} \rightarrow \ldots \rightarrow U^{k} \rightarrow A \rightarrow 0
$$

For any functor $S \in \mathcal{P}$, pre-composition with $S$ determines a new extension

$$
E(S): \quad 0 \rightarrow\left(T \otimes T^{\prime}\right)(S) \rightarrow U^{1}(S) \rightarrow \ldots \rightarrow U^{k}(S) \rightarrow A(S) \rightarrow 0
$$

(where $F(S)$ denotes the composition of the functor $F$ with $S$ ) and the corresponding element $e(S) \in \operatorname{Ext}_{\mathcal{P}}^{k}\left(A(S),\left(T \otimes T^{\prime}\right)(S)\right)$. Furthermore, any natural transformation $\phi: S \rightarrow S^{\prime}$ defines a homomorphism of extensions $E(\phi): E(S) \rightarrow E\left(S^{\prime}\right)$. The existence of such a homomorphism of extensions implies the following relation in $\operatorname{Ext}_{\mathcal{P}}^{k}\left(A(S),\left(T \otimes T^{\prime}\right)\left(S^{\prime}\right)\right)$ :

$$
\left(T \otimes T^{\prime}\right)(\phi) \cdot e(S)=e\left(S^{\prime}\right) \cdot A(\phi) .
$$

Consider the additive ("doubling") functor $D \in \mathcal{P}$ defined to send $V$ to $V \oplus V$ equipped with evident natural transformations

$$
i_{1}, i_{2}, \Delta: I \rightarrow D, p_{1}, p_{2}: D \rightarrow I
$$


Denote $e(D)$ by $e^{\prime}$. The preceding equality implies the following relations in $\operatorname{Ext}_{\mathcal{P}}^{k}\left(A, T(D) \otimes T^{\prime}(D)\right)$ :

$$
\begin{gathered}
\left(T \otimes T^{\prime}\right)(\Delta) \cdot e=e^{\prime} \cdot A(\Delta) \\
\left(T \otimes T^{\prime}\right)\left(i_{j}\right) \cdot e=e^{\prime} \cdot A\left(i_{j}\right), j=1,2 .
\end{gathered}
$$

The additivity of $A$ shows that $A(\Delta)=A\left(i_{1}\right)+A\left(i_{2}\right)$. Furthermore $T\left(p_{1} \cdot i_{2}\right)=$ $T(0)=0$ since $T$ is a homogenous functor of positive degree and also $T^{\prime}\left(p_{2} \cdot i_{1}\right)=$ $T^{\prime}(0)=0$. Finally we get:

$$
\begin{aligned}
e & =\left(T\left(p_{1}\right) \otimes T^{\prime}\left(p_{2}\right)\right) \cdot\left(T(\Delta) \otimes T^{\prime}(\Delta)\right) \cdot e=\left(T\left(p_{1}\right) \otimes T^{\prime}\left(p_{2}\right)\right) \cdot e^{\prime} \cdot A(\Delta)= \\
& =\left(T\left(p_{1}\right) \otimes T^{\prime}\left(p_{2}\right)\right) \cdot e^{\prime} \cdot A\left(i_{1}\right)+\left(T\left(p_{1}\right) \otimes T^{\prime}\left(p_{2}\right)\right) \cdot e^{\prime} \cdot A\left(i_{2}\right)= \\
& =\left(I d \otimes T^{\prime}\left(p_{2} \cdot i_{1}\right)\right) \cdot e+\left(T\left(p_{1} \cdot i_{2}\right) \otimes I d\right) \cdot e=0 .
\end{aligned}
$$

$\S 3$ Categories $\mathcal{P}_{d}$, Schur Algebras $S(n, d)$, and Rational $G L_{n}$-Modules.

As established below in Corollary 3.13, Ext-group calculations in the category $\mathcal{P}$ provide Ext-group calculations for rational $G L_{n}$-modules. This justifies our strategy of proving Theorem 1.2 (and thus Theorem 1.1) by considering extensions in the category $\mathcal{P}$.

In this section, we explain the relationship between polynomial functors of finite degree and rational $G L_{n}$-modules. In Theorem 3.2, we show that the category $\mathcal{P}_{d}$ of polynomial functors of degree $d$ is equivalent to the category of finite dimensional modules over the classical Schur algebra $S(n, d)$ provided that $n \geq d$. The remainder of this section is a comparison of Ext-groups in the category $(\mathrm{Pol})_{n}$ of polynomial $G L_{n}$-modules to Ext-groups computed in the category of rational $G L_{n}$-modules. This comparison is one that is well known to the "experts" and was apparently proved originally by J. Green. Our approach follows that of S. Donkin in $[\mathrm{D}]$.

Throughout this section, $k$ will denote an arbitrary field.

Definition 3.1. For a positive integer $n$, let $A_{n}=k\left[M_{n}\right]$ denote the Hopf algebra of algebraic functions on the $k$-vector space $M_{n}$ of $n \times n$ matrices: as an algebra, $A_{n}$ is a polynomial ring in $n^{2}$ indeterminants; the non-cocommutative comultiplication of $A_{n}$ is determined by matrix multiplication. Let $A(n, d) \subset A_{n}$ denote the subspace of homogeneous polynomials of degree $d$; so defined, $A(n, d)$ is a sub-coalgebra of $k\left[M_{n}\right]$. Finally, the Schur algebra $S(n, d)$ is the linear dual of $A(n, d), S(n, d)=$ $A(n, d)^{\#}$.

As observed by Schur (cf. [G]), this Schur algebra admits another description:

$$
\begin{gathered}
S(n, d)=\Gamma^{d}\left(\operatorname{End}_{k}\left(k^{n}\right)\right)=\left(\left(\operatorname{End}_{k}\left(k^{n}\right)\right)^{\otimes d}\right)^{\Sigma_{d}}= \\
=\left(\operatorname{End}_{k}\left(\left(k^{n}\right)^{\otimes d}\right)\right)^{\Sigma_{d}}=\operatorname{End}_{k \Sigma_{d}}\left(\left(k^{n}\right)^{\otimes d}\right) .
\end{gathered}
$$

Let $\operatorname{Mod}\{S(n, d)\}$ denote the abelian category of finitely generated left $S(n, d)$ modules. 
For any polynomial functor $T \in \mathcal{P}_{d}$ we constructed in the previous section a canonical $k$-linear map

$$
\theta_{k^{n}}: \Gamma^{d}\left(\operatorname{End}_{k}\left(k^{n}\right)\right) \otimes T\left(k^{n}\right) \rightarrow T\left(k^{n}\right) .
$$

One checks immediately that this provides $T\left(k^{n}\right)$ with the structure of a $S(n, d)$ module. Furthermore, associating to $T \in \mathcal{P}_{d}$ the corresponding module $T\left(k^{n}\right) \in$ $\operatorname{Mod}\{S(n, d)\}$ we get a functor $\Psi: \mathcal{P}_{d} \rightarrow \operatorname{Mod}\{S(n, d)\}$.

Theorem 3.2. Assume $n \geq d$. Then $\Psi: \mathcal{P}_{d} \rightarrow \operatorname{Mod}\{S(n, d)\}$ is an equivalence of categories, with quasi-inverse defined by sending $M \in \operatorname{Mod}\{S(n, d)\}$ to $\Phi(M) \equiv$ $\Gamma^{d, n} \otimes_{S(n, d)} M$.

Proof. Since $\Gamma^{d, n}(V)=\Gamma^{d}\left(\operatorname{Hom}_{k}\left(k^{n}, V\right)\right)$ has an evident structure of a right $S(n, d)$-module for any $V \in \mathcal{V}, \Phi(M)$ defined to equal $\Gamma^{d, n} \otimes_{S(n, d)} M$ is a well defined homogenous polynomial functor of degree $d$ for any $M \in \operatorname{Mod}\{S(n, d)\}$. Naturality of this construction translates to the assertion that sending $M \in M o d\{S(n, d)\}$ to $\Phi(M) \in \mathcal{P}_{d}$ determines a functor $\Phi: \operatorname{Mod}\{S(n, d)\} \rightarrow \mathcal{P}_{d}$.

The composition $\Psi \circ \Phi$ is clearly the identity functor. Furthermore one checks easily that for any polynomial functor $T \in \mathcal{P}_{d}$ the natural $k$-linear map $\theta_{V}$ : $\Gamma^{d, n}(V) \otimes T\left(k^{n}\right) \rightarrow T(V)$ factors via

$$
\tilde{\theta}_{V}: \Gamma^{d, n}(V) \otimes_{S(n, d)} T\left(k^{n}\right) \rightarrow T(V) .
$$

These maps $\widetilde{\theta}_{V}$ determine a homomorphism of functors

$$
\widetilde{\Theta}: \Gamma^{d, n} \otimes_{S(n, d)} T\left(k^{n}\right) \rightarrow T
$$

natural with respect to $T$. This homomorphism is clearly an isomorphism in case $T=\Gamma^{n, d}$ (since $T\left(k^{n}\right)=S(n, d)$ in this case). Since $\widetilde{\Theta}$ is clearly additive, we see that $\widetilde{\Theta}$ is an isomorphism for any direct sum of copies of $\Gamma^{n, d}$.

If $n \geq d$, then Theorem 2.8 implies that any $T \in \mathcal{P}_{d}$ admits a resolution of the form

$$
\left(\Gamma^{n, d}\right)^{\oplus m_{2}} \rightarrow\left(\Gamma^{n, d}\right)^{\oplus m_{1}} \rightarrow T \rightarrow 0 .
$$

We conclude that $\widetilde{\Theta}$ is an isomorphism for any such $T \in \mathcal{P}_{d}$ by appealing to the right exactness of $\Phi \circ \Psi$.

Recall that a rational $G L_{n}$-module is (by definition) a comodule for the coalgebra $k\left[G L_{n}\right]$. Since $G L_{n} \subset M_{n}$ determines a natural embeding of coalgebras $k\left[G L_{n}\right] \hookleftarrow$ $k\left[M_{n}\right]$, any $k\left[M_{n}\right]$-comodule determines a rational $G L_{n}$-module.

Definition 3.3. A rational $G L_{n}$-module $M$ is said to be polynomial if the following equivalent conditions are satisfied

(1) the comodule structure of $k\left[G L_{n}\right]$ on $M$ arises from a comodule structure of $k\left[M_{n}\right] \subset k\left[G L_{n}\right]$.

(2) the action of the algebraic group $G L_{n}$ on $M$ extends to an action of the algebraic monoid $M_{n}$.

(3) the coordinate functions of the representation of $G L_{n}$ in $M$ are polynomials in the coordinate functions on $G L_{n}$ (i.e. don't involve $1 /$ det). 
Definition 3.3.1. A polynomial $G L_{n}$-module $M$ is said to be homogenous of degree $d$ if the following equivalent conditions are satisfied

(1) the comodule structure of $k\left[G L_{n}\right]$ on $M$ arises from a comodule structure of $k\left[M_{n}\right]_{d} \subset k\left[M_{n}\right]$.

(2) $d$ is the only weight of the action of the center $\mathbf{G}_{\mathbf{m}} \subset G L_{n}$ on $M$.

(3) the coordinate functions of the representation of $G L_{n}$ on $M$ are homogenous polynomials of degree $d$ in the coordinate functions on $G L_{n}$.

We denote by $(\mathrm{Pol})_{n}$ the full subcategory of the category of rational $G L_{n^{-}}$ modules, consisting of polynomial modules and by $(\mathrm{Pol})_{n, d}$ the full subcategory of $(\mathrm{Pol})_{n}$, consisting of homogenous polynomial modules of degree $d$. Since all representations of the group $\mathbf{G}_{\mathbf{m}}$ are semisimple we conclude that every polynomial $G L_{n}$-module splits into the direct sum of its homogenous components. Since every homomorphism of rational $G L_{n}$-modules preserves weights we conclude further that this homogenous decomposition is functorial, in particular, denoting by $(P o l)_{n}^{f}$ (resp. $\left.(\mathrm{Pol})_{n, d}^{f}\right)$ the full subcategory of $(\mathrm{Pol})_{n}$ consisting of polynomial modules of finite dimension we have the following decompositions

$$
(P o l)_{n}=\prod_{d=0}^{\infty}(P o l)_{n, d}, \quad(P o l)_{n}^{f}=\bigoplus_{d=0}^{\infty}(P o l)_{n, d}^{f} .
$$

For any polynomial functor $T$ and any $n$, the rational $G L_{n}$-module $T\left(k^{n}\right)$ is clearly polynomial. Furthermore, associating to $T \in \mathcal{P}$ the polynomial $G L_{n}$-module $T\left(k^{n}\right)$ we get exact additive functors

$$
\mathcal{P} \rightarrow(P o l)_{n}^{f}, \quad \mathcal{P}_{d} \rightarrow(P o l)_{n, d}^{f}
$$

Lemma 3.4. If $n \geq d$, then the functor $\mathcal{P}_{d} \rightarrow(P o l)_{n, d}^{f}$ is an equivalence of categories.

Proof. Note that for any finite dimensional coassociative $k$-coalgebra $B$ to give a left $B$-comodule structure on a vector space $M$ is the same as to give a left $B^{\#}$ module structure on $M$ : the comultiplication $M \rightarrow B \otimes M$ defines the multiplication $B^{\#} \otimes M \rightarrow B^{\#} \otimes B \otimes M \rightarrow k \otimes M=M$ and vice versa. Thus the category of left comodules over $B$ is canonically equivalent to the category of left $B^{\#}$-modules. Applying this remark to the coalgebra $k\left[M_{n}\right]_{d}$ we get a canonical equivalence of categories $(P o l)_{n, d}^{f} \rightarrow \operatorname{Mod}\{S(n, d)\}$. Now our statement follows from Theorem 3.2 and the commutativity of the following diagram of functors

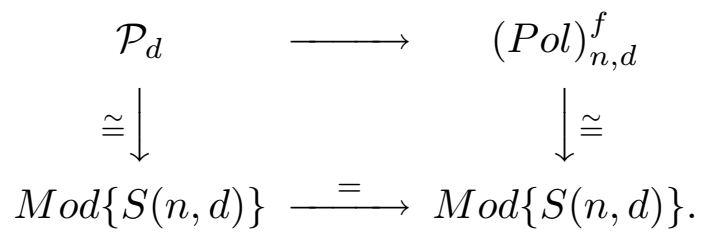


Corollary 3.4.1. If $n \geq d$, then for any two homogenous polynomial functors $S, T \in \mathcal{P}_{d}$ the exact functor $\mathcal{P} \rightarrow(P o l)_{n}$ sending $T$ to $T\left(k^{n}\right)$ induces isomorphisms

$$
\operatorname{Ext}_{\mathcal{P}}^{*}(S, T) \stackrel{\sim}{\longrightarrow} \operatorname{Ext}_{(P o l)_{n}}^{*}\left(S\left(k^{n}\right), T\left(k^{n}\right)\right) .
$$

Proof. This follows immediately from Lemma 3.4 and the obvious fact that inclusions of categories $(\mathrm{Pol})_{n, d}^{f} \hookrightarrow(\mathrm{Pol})_{n, d} \hookrightarrow(\mathrm{Pol})_{n}$ induce isomorphisms on Extgroups ( for the first inclusion use the local finiteness of rational modules).

In the remainder of this section we are going to consider the category of rational modules over the reductive group $G L_{n}$. Let $T_{n}=$ the subgroup of diagonal matrices $\}$ denote the standard maximal torus of $G L_{n}$, let further $B_{n}=\{$ the subgroup of upper triangular matrices $\}$ and $B_{n}^{+}=\{$the subgroup of lower triangular matrices\} be two standard Borel subgroups of $G L_{n}$. We denote by $\Lambda=X\left(T_{n}\right)$ the weight lattice of $G L_{n}$; the elements of $\Lambda$ will be usually identified with sequences $\lambda=\left(\lambda_{1}, \ldots, \lambda_{n}\right)$ of integers. We denote by $\epsilon_{i}=(0, \ldots, 1, \ldots, 0)$ the $i^{t h}$ standard basis element of $\Lambda$. We denote further by $\Phi \subset \Lambda$ the root system of $G L_{n}$. Thus $\Phi=\left\{\left(\epsilon_{j}-\epsilon_{i}\right)_{1 \leq i \neq j \leq n}\right\}$. We let $\Phi^{+}=\left\{\left(\epsilon_{j}-\epsilon_{i}\right)_{1 \leq i<j \leq n}\right\}$ be the system of positive roots corresponding to the Borel subgroup $B_{n}^{+}$. Let finally $\Lambda^{+} \subset \Lambda$ denote the subset of dominant weights. Thus $\Lambda^{+}=\left\{\lambda=\left(\lambda_{1}, \ldots, \lambda_{n}\right):\left(\lambda, \epsilon_{j}-\epsilon_{i}\right) \geq\right.$ $0 \quad$ for any $j>i\}=\left\{\lambda=\left(\lambda_{1}, \ldots, \lambda_{n}\right): \lambda_{1} \leq \ldots \leq \lambda_{n}\right\}$. We write $\lambda \geq \mu$ provided that $\lambda-\mu=\sum_{j>i} m_{i, j}\left(\epsilon_{j}-\epsilon_{i}\right)$ with each $m_{i, j} \geq 0$ (i.e. $\lambda-\mu \in \mathbf{Z}^{+} . \Phi^{+}$). We identify the Weyl group of $G L_{n}$ with $\Sigma_{n}$.

Proposition 3.5. A rational $G L_{n}$-module $M$ is polynomial if and only if all the weights $\lambda=\left(\lambda_{1}, \ldots, \lambda_{n}\right)$ of $M$ satisfy the condition that each $\lambda_{i} \geq 0$.

Proof. Assume that $M$ is a polynomial $G L_{n}$-module. Let $\lambda$ be a weight of $M$ and let $e \in M$ be the corresponding weight vector. Complete $e$ to a basis of $M$. Note that (in this basis) $\lambda$ is a coordinate function of the restriction of the given representation of $G L_{n}$ to the maximal torus $T_{n}$. We conclude now from Definition 3.3 that

$$
\lambda\left(\operatorname{diag}\left(x_{1}, \ldots, x_{n}\right)\right)=x_{1}^{\lambda_{1}} \cdot \ldots \cdot x_{n}^{\lambda_{n}}
$$

is a polynomial function in $x_{i}$ and hence $\lambda_{i} \geq 0$ for all $i$.

Conversely, assume that all weights appearing in $M$ have non-negative coordinates. In proving that $M$ is polynomial we may (and will) assume that $M$ is finite dimensional over $k$. Choose a basis for $M$ consisting of weight vectors and write the representation of $G L_{n}$ in $M$ in the form $\alpha \mapsto f(\alpha)=g(\alpha) /(\text { det } \alpha)^{k}$, where $g(\alpha)=\left(g_{i j}(\alpha)\right)_{i, j=1}^{N}$ is a matrix depending polynomially on $\alpha$. Proceeding by induction on $k$ it suffices to show that if $k>0$ then all polynomials $g_{i j}$ are divisible by det. Since the polynomial det is irreducible it suffices to show that $g(\alpha)=0$ provided that $\alpha \in M_{n}(\bar{k})$ and $\operatorname{det}(\alpha)=0$. This is clear if $\alpha$ is a diagonal matrix. To settle the general case note that the equation $g(\beta \cdot \gamma)=g(\beta) \cdot g(\gamma)$ holds for any $\beta, \gamma \in G L_{n}(\bar{k})$. Since both sides of this equation depend polynomially on $\beta$ and $\gamma$ we conclude that this equation holds for any $\beta, \gamma \in M_{n}(\bar{k})$. Any matrix $\alpha \in M_{n}(\bar{k})$ may be written in the form $\alpha=\beta \cdot \alpha_{0} \cdot \gamma$, where $\beta, \gamma \in G L_{n}(\bar{k})$ and the matrix $\alpha_{0}$ is diagonal. Now if $\operatorname{det}(\alpha)=0$ then $\operatorname{det}\left(\alpha_{0}\right)=0$ as well and hence $g(\alpha)=g(\beta) \cdot g\left(\alpha_{0}\right) \cdot g(\gamma)=0$. 
Corollary 3.5.1. Let $M$ be a finite dimensional rational $G L_{n}$-module. Then $M$ is polynomial if and only if all maximal weights $\mu=\left(\mu_{1}, \ldots, \mu_{n}\right)$ of $M$ satisfy the condition $\mu_{1} \geq 0$.

Proof. Assume that the above condition is satisfied. Let $\lambda=\left(\lambda_{1}, \ldots, \lambda_{n}\right)$ be any weight of $M$ and let $\mu$ be a maximal weight such that $\mu \geq \lambda$. Note that inequality $\mu \geq \lambda$ implies that $\mu_{1} \leq \lambda_{1}$ and hence $\lambda_{1} \geq 0$. Thus all weights $\lambda$ of $M$ satisfy $\lambda_{1} \geq 0$. On the other hand the set of weights of any module is stable with respect to the action of the Weyl group $\Sigma_{n}$. Applying the above inequality to the weights $\sigma \lambda \quad\left(\sigma \in \Sigma_{n}\right)$ we conclude that $\lambda_{i} \geq 0$ for all $i$.

Corollary 3.6. If

$$
0 \rightarrow M^{\prime} \rightarrow M \rightarrow M^{\prime \prime} \rightarrow 0
$$

is a short exact sequence of rational $G L_{n}$-modules, then $M$ is polynomial if and only if both $M^{\prime}$ and $M^{\prime \prime}$ are polynomial.

Proof. This follows from Proposition 3.5 and the observation that $\lambda$ is a weight of $M$ (i.e., the $\lambda$ weight-space of $M$ is non-zero) if and only if $\lambda$ is a weight of either $M^{\prime}$ or $M^{\prime \prime}$.

Corollary 3.7. Sending a rational $G L_{n}$-module $M$ to the largest polynomial submodule $\mathcal{G}(M) \subset M$ determines a left-exact functor

$$
\mathcal{G}:\left(G L_{n} \text {-modules }\right) \rightarrow(P o l)_{n}
$$

from the category of rational $G L_{n}$-modules to the category of polynomial $G L_{n^{-}}$ modules. The functor $\mathcal{G}$ is right adjoint to the inclusion functor $(P o l)_{n} \hookrightarrow\left(G L_{n}\right.$-modules $)$.

Proof. By Corollary 3.6, the sum of all polynomial submodules of $M$ is again polynomial, so that $\mathcal{G}$ is well-defined. The functoriality of $\mathcal{G}$ follows from the fact that the image of a polynomial module is again polynomial (by Corollary 3.6 once again). The properties of $\mathcal{G}$ are clear from definitions and Corollary 3.6.

For any dominant weight $\lambda \in \Lambda^{+}$we denote by $S(\lambda)$ the irreducible $G L_{n}$-module of high weight $\lambda$. We denote further by $h_{0}(\lambda)$ the rational $G L_{n}$-module $\left.k_{\lambda}\right|^{G L_{n}}$ induced from the 1-dimensional $B_{n}$-module $k_{\lambda}$ determined by $\lambda$. Thus $S(\lambda)$ may be identified with the socle of $h_{0}(\lambda)$ (see $[\mathrm{J}]$ ). Finally we denote by $V(\lambda)$ the Weyl module $h_{0}\left(-w_{0} \lambda\right)^{\#}$, where $w_{0}$ is the element of the Weyl group taking $B_{n}$ to $B_{n}^{+}$ (i.e. $w_{0}$ corresponds to the permutation $i \mapsto n+1-i$ ).

Let $\Pi \subset \Lambda^{+}$be the subset consisting of those dominant weights $\lambda$ which satisfy the condition $\lambda_{1} \geq 0$ and let $\bar{\Pi}=\Lambda^{+}-\Pi$ be the complement of $\Pi$. Note that $\Pi$ is saturated in the sense that if $\lambda \leq \mu$ are dominant weights and $\mu \in \Pi$ then $\lambda \in \Pi$ as well.

\section{Lemma 3.8.}

(1) If $\lambda \in \Pi$ then the modules $S(\lambda)$ and $h_{0}(\lambda)$ are polynomial and hence

$$
\mathcal{G}(S(\lambda))=S(\lambda), \quad \mathcal{G}\left(h_{0}(\lambda)\right)=h_{0}(\lambda) .
$$

(2) If $\lambda \in \bar{\Pi}$ then

$$
\mathcal{G}(S(\lambda))=0, \quad \mathcal{G}\left(h_{0}(\lambda)\right)=0
$$


Proof. The point (1) follows immediately from Corollary 3.5.1 since $\lambda$ is the unique maximal weight of both $S(\lambda)$ and $h_{0}(\lambda)$ (see [J;II.2.2]).

If $\lambda \in \bar{\Pi}$ then $S(\lambda)$ is not polynomial according to Proposition 3.5. Hence $S(\lambda) \not \subset \mathcal{G}\left(h_{0}(\lambda)\right)$. On the other hand every nonzero submodule of $h_{0}(\lambda)$ contains $S(\lambda)$. Thus $\mathcal{G}(S(\lambda)) \subset \mathcal{G}\left(h_{0}(\lambda)\right)=0$.

We recall that a good filtration on the module $M$ is a filtration $0=M_{0} \subset M_{1} \subset$ $\ldots \subset M$ such that $M=\bigcup_{i} M_{i}$ and all subsequent factors $M_{i} / M_{i-1}$ of this filtration are of the form $h_{0}(\lambda)$. We will need the following usefull criterion for the existence of the good filtration for which we refer the reader to $[\mathrm{F}]$.

\section{Lemma 3.9.}

(1) If $M$ admits a good filtration, then the dimension of $M$ is at most countable and for any $\lambda \in \Lambda^{+}$we have:

$$
\operatorname{dim} \operatorname{Hom}_{G L_{n}}(V(\lambda), M)=\left[M: h_{0}(\lambda)\right], \quad \operatorname{Ext}_{G L_{n}}^{i}(V(\lambda), M)=0 \text { for } i>0
$$

where $\left[M: h_{0}(\lambda)\right]$ is the number of factors isomorphic to $h_{0}(\lambda)$ in any good filtration of $M$.

(2) If dimension of $M$ is at most countable and $\operatorname{Ext}_{G L_{n}}^{1}(V(\lambda), M)=0$ for all $\lambda \in \Lambda^{+}$, then $M$ admits a good filtration

Lemma 3.10. If $\lambda, \mu \in \Lambda^{+}$and $\lambda \ngtr \mu$, then $\operatorname{Ext}_{G L_{n}}^{i}\left(h_{0}(\lambda), h_{0}(\mu)\right)=0$ for all $i>0$.

Proof. This fact is proved for any semisimple group in[CPSK;3.2] and the proof applies to any reductive group as well.

Corollary 3.10.1. Let $M$ be a finite dimensional rational $G L_{n}$-module with a good filtration. Then there exists a good filtration $0=M_{0} \subset M_{1} \subset \ldots \subset M_{l}=M$ and an integer $k$ such that $M_{i} / M_{i-1}=h_{0}\left(\lambda_{i}\right)$ with $\lambda_{i} \in \Pi$ for $1 \leq i \leq k$ and $\lambda_{i} \in \bar{\Pi}$ for $k+1 \leq i \leq l$.

Proof. Choose any good filtration $0=M_{0} \subset \ldots \subset M_{l}=M$. Assume that there exists an integer $i$ such that $\lambda_{i} \in \Pi$ and $\lambda_{i-1} \in \bar{\Pi}$. According to Lemma 3.10 $\operatorname{Ext}_{G L_{n}}^{1}\left(h_{0}\left(\lambda_{i}\right), h_{0}\left(\lambda_{i-1}\right)=0\right.$. This shows that $M_{i} / M_{i-2}=h_{0}\left(\lambda_{i}\right) \oplus h_{0}\left(\lambda_{i-1}\right)$ and allows us to interchange the factors $h_{0}\left(\lambda_{i}\right)$ and $h_{0}\left(\lambda_{i-1}\right)$. It's clear that applying this procedure several times we come to a good filtration with the desired properties.

Corollary 3.10.2. Under the conditions and notation of Corollary 3.10.1, $\mathcal{G}(M)=$ $M_{k}$. In particular $\mathcal{G}(M)$ has a good filtration and

$$
\left[\mathcal{G}(M): h_{o}(\lambda)\right]=\left\{\begin{array}{l}
{\left[M: h_{o}(\lambda)\right] \quad \text { if } \quad \lambda \in \Pi} \\
0 \quad \text { otherwise. }
\end{array}\right.
$$

Proof. Lemma 3.8 and Corollary 3.6 show that the module $M_{k}$ is polynomial. On the other hand $\mathcal{G}\left(M / M_{k}\right)=0$ according to Lemma 3.8 and left exactness of the functor $\mathcal{G}$. 
Proposition 3.11. Let $0 \rightarrow M^{\prime} \rightarrow M \rightarrow M^{\prime \prime} \rightarrow 0$ be a short exact sequence of rational $G L_{n}$-modules. Assume that both $M^{\prime}$ and $M^{\prime \prime}$ have good filtrations. Then the sequence $0 \rightarrow \mathcal{G}\left(M^{\prime}\right) \rightarrow \mathcal{G}(M) \rightarrow \mathcal{G}\left(M^{\prime \prime}\right) \rightarrow 0$ is exact.

Proof. If both $M^{\prime}$ and $M^{\prime \prime}$ are finite dimentional then our statement follows (by counting dimensions) from Corollary 3.10.2. Assume now that only $M^{\prime \prime}$ is finite dimensional. Choose any good filtration $0=M_{0}^{\prime} \subset M_{1}^{\prime} \subset \ldots$ of $M^{\prime}$. Since $M^{\prime \prime}$ is finite dimensional we conclude that $\operatorname{Ext}_{G L_{n}}^{1}\left(M^{\prime \prime}, M^{\prime}\right)=\varliminf_{i} \operatorname{Ext}_{G L_{n}}^{1}\left(M^{\prime \prime}, M_{i}^{\prime}\right)$. Thus there exists an index $i$ such that our extension may be obtained by means of the cobase change from a certain extension $0 \rightarrow M_{i}^{\prime} \rightarrow M_{i} \rightarrow M^{\prime \prime} \rightarrow 0$. Since $M_{i}^{\prime}$ is finite dimensional we conclude that the map $\mathcal{G}\left(M_{i}\right) \rightarrow \mathcal{G}\left(M^{\prime \prime}\right)$ is surjective. Hence the map $\mathcal{G}(M) \rightarrow \mathcal{G}\left(M^{\prime \prime}\right)$ is surjective as well. In the general case choose a good filtration $0=M_{0}^{\prime \prime} \subset M_{1}^{\prime \prime} \subset \ldots$ of $M^{\prime \prime}$. It suffices to show that $\mathcal{G}\left(M_{i}^{\prime \prime}\right)$ is contained in the image of $\mathcal{G}(M)$ and this follows applying the already proved result to the extension of $M_{i}^{\prime \prime}$ by means of $M^{\prime}$ obtained from our original one by means of the base change $M_{i}^{\prime \prime} \hookrightarrow M^{\prime \prime}$.

The following theorem (and proof) constitute our interpretation S. Donkin's proof of Green's Theorem (cf.[D;2.1f]) .

Theorem 3.12. The higher right derived functors of the left exact functor $\mathcal{G}$ : $\left(G L_{n}\right.$-modules $) \rightarrow(P o l)_{n}$ vanish on all rational $G L_{n}$-modules which either have a good filtration or are polynomial (i.e., lie in $\left.(\mathrm{Pol})_{n}\right)$.

Proof. Let $M$ be any module with a good filtration. Embed $M$ in an injective module $I=M_{\operatorname{tr}} \otimes k\left[G L_{n}\right]$ and denote $I / M$ by $M^{\prime}$. Note that $M^{\prime}$ also admits a good filtration. In fact dimension of $M^{\prime}$ is clearly (at most) countable and $\operatorname{Ext}^{1}\left(V(\mu), M^{\prime}\right)=\operatorname{Ext}^{2}(V(\mu), M)=0$ for all $\mu \in \Lambda^{+}$, so that we may apply Lemma 3.9. Proposition 3.11 implies now that the sequence

$$
0 \rightarrow \mathcal{G}(M) \rightarrow \mathcal{G}(I) \rightarrow \mathcal{G}\left(M^{\prime}\right) \rightarrow 0
$$

is exact, so that $R^{1} \mathcal{G}(M)=0$ and $R^{i} \mathcal{G}(M)=R^{i-1} \mathcal{G}\left(M^{\prime}\right)$ for $i>1$. Thus, a simple induction ends the argument provided that $M$ has a good filtration.

Devissage and a direct limit argument verifies that $R^{j} \mathcal{G}(M)=0$ for all $0<j \leq t$ and all polynomial representations $M$ if and only if $R^{j} \mathcal{G}(S(\lambda))=0$ for all irreducible polynomial representation $S(\lambda)$ and all $0<j \leq t$. Consider the short exact sequence

$$
0 \rightarrow S(\lambda) \rightarrow h_{0}(\lambda) \rightarrow T(\lambda) \rightarrow 0
$$

with $S(\lambda)$ polynomial (i.e., $\lambda \in \Pi$ ). Lemma 3.8 implies that $h_{0}(\lambda)$ is polynomial; by Corollary 3.6, $T(\lambda)$ is also polynomial. Since $\mathcal{G}$ is the identity on polynomial modules, the vanishing of $R^{1} \mathcal{G}\left(h_{0}(\lambda)\right)$ together with the long exact sequence of derived functors of $\mathcal{G}$ implies the vanishing of $R^{1} \mathcal{G}(S(\lambda))$. Thus, $R^{1} \mathcal{G}(T(\lambda))=0$ as well. We now use the long exact sequence of derived functors of $\mathcal{G}$ to complete an inductive proof of the vanishing of $R^{j} \mathcal{G}\left(S(\lambda)\right.$ ) (and thus of $R^{j} \mathcal{G}(M)$ for all polynomial modules $M$ ) for $j>0$. 
Corollary 3.12.1. The embeding of categories

$$
(\text { Pol })_{n} \hookrightarrow\left(\text { rational } G L_{n}-\text { modules }\right)
$$

induces isomorphisms on the Ext-groups.

Proof. This follows from Theorem 3.12 since $\mathcal{G}$ is right adjoint to (as well as left inverse to) the embeding functor.

Corollary 3.13. Let $T$ and $T^{\prime}$ be homogenous polynomial functors of degree $d$. Then the exact functor $\mathcal{P} \rightarrow\left(G L_{n}\right.$-modules $): T \mapsto T\left(k^{n}\right)$ induces isomorphisms

$$
\operatorname{Ext}_{\mathcal{P}}^{*}\left(T, T^{\prime}\right) \stackrel{\sim}{\longrightarrow} \operatorname{Ext}_{G L_{n}}^{*}\left(T\left(k^{n}\right), T^{\prime}\left(k^{n}\right)\right)
$$

provided that $n \geq d$.

Proof. This follows immediately from Corollary 3.4.1 and Corollary 3.12.1.

Remark. Corollary 3.13 is a precise stability result with respect to $n$ for the rational Ext-groups of certain coherent families of rational $G L_{n}$-modules.

Let $T$ and $T^{\prime}$ be two polynomial functors. For each $n$ the exact functor $\mathcal{P} \rightarrow$ (rational $G L_{n}$-modules) : $T \mapsto T\left(k^{n}\right)$ induces natural homomorphisms

$$
\operatorname{Ext}_{\mathcal{P}}^{*}\left(T, T^{\prime}\right) \rightarrow \operatorname{Ext}_{G L_{n}}^{*}\left(T\left(k^{n}\right), T^{\prime}\left(k^{n}\right)\right) .
$$

We would like to show that these homomorphisms are compatible one with another in an appropriate sense. Assume that $n \leq m$. We consider the standard (into the left upper corner) embeding $G L_{n} \hookrightarrow G L_{m}$, we also denote by $i: k^{n} \hookrightarrow k^{m}$ and $p: k^{m} \rightarrow k^{n}$ the standard $k$-linear embeding and projection. For any polynomial functor $T$ the vector space $T\left(k^{m}\right)$ is a (polynomial) $G L_{m}$-module and hence may be considered as a (polynomial) $G L_{n}$-module via the embeding $G L_{n} \hookrightarrow G L_{m}$. One checks immediately that $T(i): T\left(k^{n}\right) \rightarrow T\left(k^{m}\right)$ and $T(p): T\left(k^{m}\right) \rightarrow T\left(k^{n}\right)$ are homomorphisms of $G L_{n}$-modules. For any polynomial functors $T$ and $T^{\prime}$ and any $n \leq m$ this determines restriction homomorphisms

$$
\operatorname{Ext}_{G L_{m}}^{*}\left(T\left(k^{m}\right), T^{\prime}\left(k^{m}\right)\right) \rightarrow \operatorname{Ext}_{G L_{n}}^{*}\left(T\left(k^{m}\right), T^{\prime}\left(k^{m}\right)\right) \rightarrow \operatorname{Ext}_{G L_{n}}^{*}\left(T\left(k^{n}\right), T^{\prime}\left(k^{n}\right)\right) .
$$

Lemma 3.14. For any polynomial functors $T, T^{\prime}$ and any $n \leq m$ the following diagram commutes

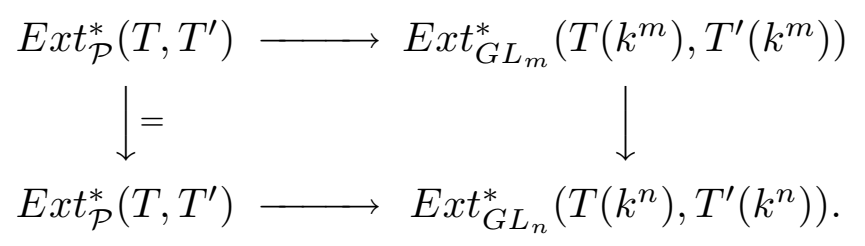

Proof. Let $e \in \operatorname{Ext}_{\mathcal{P}}^{l}\left(T, T^{\prime}\right)$ be represented by an extension

$$
E: \quad 0 \rightarrow T^{\prime} \rightarrow U^{1} \rightarrow \ldots \rightarrow U^{l} \rightarrow T \rightarrow 0
$$


of polynomial functors. The image of $e$ in $\operatorname{Ext}_{G L_{n}}^{l}\left(T\left(k^{n}\right), T^{\prime}\left(k^{n}\right)\right)$ is represented by the extension

$$
E\left(k^{n}\right): \quad 0 \rightarrow T^{\prime}\left(k^{n}\right) \rightarrow U^{1}\left(k^{n}\right) \rightarrow \ldots \rightarrow U^{l}\left(k^{n}\right) \rightarrow T\left(k^{n}\right) \rightarrow 0
$$

of rational $G L_{n}$-modules and the image of $e$ in $\operatorname{Ext}_{G L_{m}}^{l}\left(T\left(k^{m}\right), T^{\prime}\left(k^{m}\right)\right)$ is represented by the extension $E\left(k^{m}\right)$ of rational $G L_{m}$-modules. Furthermore the image of $\left[E\left(k^{m}\right)\right] \in \operatorname{Ext}_{G L_{m}}^{l}\left(T\left(k^{m}\right), T^{\prime}\left(k^{m}\right)\right)$ in $\operatorname{Ext}_{G L_{n}}^{l}\left(T\left(k^{n}\right), T^{\prime}\left(k^{n}\right)\right)$ is equal, according to the definition above, to $T^{\prime}(p) \cdot\left[\left.E\left(k^{m}\right)\right|_{G L_{n}}\right] \cdot T(i)$. Note finally that we have a homomorphism of extensions

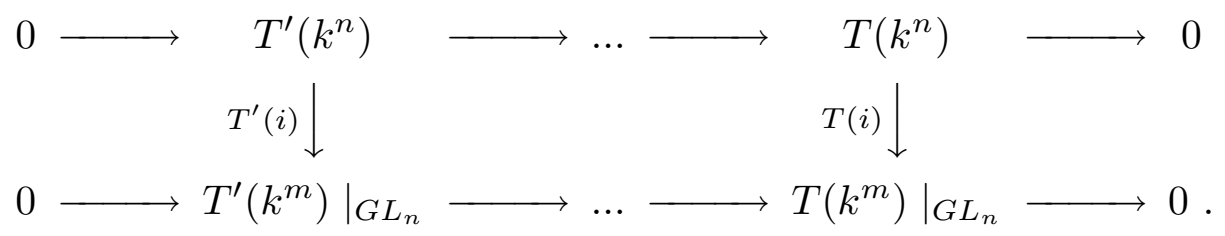

The existence of such a homomorphism gives us the relation $\left[\left.E\left(k^{m}\right)\right|_{G L_{n}}\right] \cdot T(i)=$ $T^{\prime}(i) \cdot\left[E\left(k^{n}\right)\right]$, so that $T^{\prime}(p) \cdot\left[\left.E\left(k^{m}\right)\right|_{G L_{n}}\right] \cdot T(i)=T^{\prime}(p) \cdot T^{\prime}(i) \cdot\left[E\left(k^{n}\right)\right]=\left[E\left(k^{n}\right)\right]$.

\section{§4. Computation of $\operatorname{Ext}_{\mathcal{P}}^{*}\left(I^{(r)}, S^{p^{r-j}(j)}\right)$.}

In this section we construct the rational cohomology classes

$$
e_{r} \in H^{2 p^{r-1}}\left(G L_{n}, g l_{n}^{(r)}\right)
$$

Following further analysis of Ext-groups in the category $\mathcal{P}$ achieved in section 5 , non-vanishing of the restriction of $e_{r}$ to $\left(G L_{n}\right)_{(1)}$ is verified in section 6 . In this section $k$ will denote a field of characteristic $p>0$.

We shall let $I \in \mathcal{P}_{1}$ denote the identity functor and $I^{(r)} \in \mathcal{P}_{p^{r}}$ the $r$-th Frobenius twist of $I$. Then $I^{(r)}\left(k^{n}\right)$ is the polynomial $G L_{n}$-module obtained by applying the $r$-th Frobenius twist to the standard representation of $G L_{n}$. Thus

$$
I^{(r)}\left(k^{n}\right)^{\#} \otimes I^{(r)}\left(k^{n}\right)=g l_{n}^{(r)}
$$

as rational $G L_{n}$-modules. To construct $e_{r}$, it suffices in view of the existence of a natural homomorphism

$$
\operatorname{Ext}_{\mathcal{P}}^{2 p^{r-1}}\left(I^{(r)}, I^{(r)}\right) \rightarrow \operatorname{Ext}_{G L_{n}}^{2 p^{r-1}}\left(I^{(r)}\left(k^{n}\right), I^{(r)}\left(k^{n}\right)\right)=H^{2 p^{r-1}}\left(G L_{n}, g l_{n}^{(r)}\right)
$$

(which is an isomorphism for $n \geq p^{r}$ ) to compute the group $\operatorname{Ext}_{\mathcal{P}}^{2 p^{r-1}}\left(I^{(r)}, I^{(r)}\right)$.

Following the work of V. Franjou, J. Lannes, and L. Schwartz in [FLS], we achieve this computation by computing $\operatorname{Ext}_{\mathcal{P}}^{*}\left(I^{(r)}, S^{p^{r-j}(j)}\right)$ for $0 \leq j \leq r$. In particular, the special case $j=r$ gives us the computation we seek, whereas the special case $j=0$ follows from the injectivity of $S^{d}$ for any $d$ (since $S^{d}$ is the dual of $\Gamma^{d}=\Gamma^{d, 1}$ whose projectivity is given by Theorem 2.10). Thus, induction with respect to $j$ is naturally suggested. 
The key technical tool that facilitates our computation is the vanishing result of Theorem 2.13. Indeed, the arguments in [FLS] apply almost without change to our context of the category $\mathcal{P}$. In this section, we present a somewhat streamlined version of the arguments of Franjou-Lannes-Schwartz for the category $\mathcal{P}$. Following [FLS], we begin by recalling several useful complexes.

For any vector space $V \in \mathcal{V}$, set

$$
\Omega_{n}^{i}(V)=S^{n-i}(V) \otimes \Lambda^{i}(V), \Omega_{*}^{*}(V)=\bigoplus_{n \geq i \geq 0} \Omega_{n}^{i}(V) .
$$

We shall often refer to $i$ as the cohomological degree, and to $n$ as the total degree.

Note that $\Omega_{*}^{*}(V)$ has a natural structure of a graded commutative (with respect to the cohomological degree) algebra. This algebra may be equipped with two natural differentials, each of which is a graded derivation: the De Rham differential $d$ and the Koszul differential $\kappa$. Both these differentials preserve the total degree, $d$ increases and $\kappa$ decreases the cohomological degree by one. They are defined in terms of the product and coproduct operations as follows:

$$
\begin{aligned}
d: \Omega_{n}^{i}(V) & =S^{n-i}(V) \otimes \Lambda^{i}(V) \rightarrow S^{n-i-1}(V) \otimes V \otimes \Lambda^{i}(V) \rightarrow \\
& \rightarrow S^{n-i-1}(V) \otimes \Lambda^{i+1}(V)=\Omega_{n}^{i+1}(V) \\
\kappa: \Omega_{n}^{i}(V) & =S^{n-i}(V) \otimes \Lambda^{i}(V) \rightarrow S^{n-i}(V) \otimes V \otimes \Lambda^{i-1}(V) \rightarrow \\
& \rightarrow S^{n-i+1}(V) \otimes \Lambda^{i-1}(V)=\Omega_{n}^{i-1}(V) .
\end{aligned}
$$

The homology of the De Rham complex is given by the following theorem of Cartier $[\mathrm{C}]$. Note that since $d$ is a derivation the homology $H^{*}\left(\left(\Omega_{*}^{*}(V), d\right)\right)$ is a bigraded, graded commutative (with respect to the cohomological degree) algebra. Furthermore we have canonical homomorphisms

$\Omega_{1}^{0}\left(V^{(1)}\right)=V^{(1)} \rightarrow H^{0}\left(\left(\Omega_{p}^{*}(V), d\right)\right) \quad v^{(1)} \mapsto$ homology class of the cycle $v^{p}$

$\Omega_{1}^{1}\left(V^{(1)}\right)=V^{(1)} \rightarrow H^{1}\left(\left(\Omega_{p}^{*}(V), d\right)\right) \quad v^{(1)} \mapsto$ homology class of the cycle $v^{p-1} \otimes d v$

Theorem 4.1 (Cartier $[\mathrm{C}]$ ). The induced map of algebras

$$
\Omega_{*}^{*}\left(V^{(1)}\right) \rightarrow H^{*}\left(\left(\Omega_{*}^{*}(V), d\right)\right)
$$

is an isomorphism.

Note that the Cartier isomorphism preserves the cohomological degree but multiplies the total degree by $p$. The De Rham complex is a direct sum of its homogenous (with respect to the total degree) components which we denote by $\Omega_{n}^{\bullet}(V)$. The theorem of Cartier shows that $\Omega_{n}^{\bullet}(V)$ is acyclic provided that $n \not \equiv 0 \bmod p$. Actually in this case the De Rham complex is contractible as one sees from the following Lemma

Lemma 4.2. $d \kappa+\kappa d=n$ on the complex $\Omega_{n}^{\bullet}(V)$.

Following [FLS], we shall employ the following "Koszul kernel subcomplex" $K_{p n}^{\bullet}$ of the DeRham complex. Define $K_{n}^{i}(V)$ by

$$
K_{n}^{i}(V) \stackrel{\text { def }}{=} \operatorname{ker}\left\{\kappa: \Omega_{n}^{i}(V) \rightarrow \Omega_{n}^{i-1}(V)\right\} .
$$

Lemma 4.2 shows that $K_{p n}^{\bullet}(V)$ is a subcomplex of $\Omega_{p n}^{\bullet}(V)$. Moreover we have the following easy fact 
Lemma 4.3 ([FLS]). The Cartier isomorphism restricts to give an isomorphism

$$
K_{n}^{*}\left(V^{(1)}\right) \stackrel{\sim}{\longrightarrow} H^{*}\left(K_{p n}^{\bullet}(V)\right) .
$$

Note that all the above constructions are clearly functorial in $V$, so that we may, in particular, consider the complex $\Omega_{n}^{\bullet}$ of homogenous polynomial functors of degree $n$.

For any $T \in \mathcal{P}_{n}$, we set $T^{(1)}=T \circ I^{(1)} \in \mathcal{P}_{p n}, \ldots, T^{(r)}=T^{(r-1)} \circ I^{(1)} \in \mathcal{P}_{p^{r} n}$. It's clear that $T \mapsto T^{(r)}$ is an exact additive functor from the category $\mathcal{P}_{n}$ to $\mathcal{P}_{p^{r} n}$. In particular for any pair $T, T^{\prime} \in \mathcal{P}_{n}$ we get a canonical homomorphism $\operatorname{Ext}_{\mathcal{P}}^{*}\left(T, T^{\prime}\right) \rightarrow \operatorname{Ext}_{\mathcal{P}}^{*}\left(T^{(r)},\left(T^{\prime}\right)^{(r)}\right)$. We will use the same notation $\lambda \mapsto \lambda^{(r)}$ for this homomorphism.

Remark. One could also define the Frobenius twist for functors multiplying by $I^{(1)}$ on the left instead of multiplying on the right. Luckily when dealing with functors and functor homomorphisms defined over the prime field $\mathbf{F}_{p}$ (which will always be the case below) this gives the same answer (see the proof of Theorem 4.10).

As is well known, the Koszul complex $K o_{n}^{\bullet}=\left(\Omega_{n}^{*}, \kappa\right)$ is acyclic. Following [FLS] once again, we use this property to obtain the following relations among Ext-groups.

Proposition 4.4. For any $j, 0 \leq j \leq r$, and any $i, 0 \leq i<p^{r-j}$

$$
\operatorname{Ext}_{\mathcal{P}}^{*}\left(I^{(r)}, S^{p^{r-j}(j)}\right)=\operatorname{Ext}_{\mathcal{P}}^{*+i}\left(I^{(r)}, K_{p^{r-j}}^{i(j)}\right)=\operatorname{Ext}_{\mathcal{P}}^{*+p^{r-j}-1}\left(I^{(r)}, \Lambda^{p^{r-j}(j)}\right) .
$$

Proof. The acyclicity of the Koszul complex provides short exact sequences in $\mathcal{P}$

$$
0 \rightarrow K_{p^{r-j}}^{i(j)} \rightarrow \Omega_{p^{r-j}}^{i(j)} \rightarrow K_{p^{r-j}}^{i-1(j)} \rightarrow 0 .
$$

Thus, the asserted relations follow from the following equalities:

$$
\begin{gathered}
K_{p^{r-j}}^{0(j)}=\Omega_{p^{r-j}}^{0(j)}=S^{p^{r-j}(j)} \\
K_{p^{r-j}}^{p^{r-j}-1(j)}=\Omega_{p^{r-j}}^{p^{r-j}(j)}=\Lambda^{p^{r-j}(j)}
\end{gathered}
$$

and the vanishing given by Theorem 2.13

$$
\operatorname{Ext}_{\mathcal{P}}^{*}\left(I^{(r)}, \Omega_{p^{r-j}}^{i(j)}\right)=0 \quad i \neq 0, p^{r-j} .
$$

Theorem 4.5. For any $j, 0 \leq j \leq r$, we have the following computation of Extgroups:

$$
\operatorname{Ext}_{\mathcal{P}}^{q}\left(I^{(r)}, S^{p^{r-j}(j)}\right)= \begin{cases}k & \text { if } q \equiv 0 \bmod 2 p^{r-j}, q<2 p^{r} \\ 0 & \text { otherwise. }\end{cases}
$$

Proof. We proceed by induction on $j$. The case $j=0$ follows from the injectivity of $S^{p^{r}}$ (cf. Theorem 2.10) and the identification $\operatorname{Hom}_{\mathcal{P}}\left(I^{(r)}, S^{p^{r}}\right)=I^{(r)}(k)^{\#}=k$ (cf. Theorem 2.10). Assume that the statement of the theorem is true for $j<r$. We proceed to show that it is also true for $j+1$. The proof will be divided into several steps. The following statement is immediate from Proposition 4.4. 
(4.5.1). We have the following computation of the Ext-groups

$$
\operatorname{Ext}_{\mathcal{P}}^{q}\left(I^{(r)}, \Lambda^{p^{r-j}(j)}\right)= \begin{cases}k & \text { if } q \equiv p^{r-j}-1 \bmod 2 p^{r-j}, q<2 p^{r} \\ 0 & \text { otherwise. }\end{cases}
$$

(4.5.2). For any $q$ we have the following short exact sequence (at least one of the end terms of which is zero

$$
\begin{array}{r}
0 \rightarrow \operatorname{Ext}_{\mathcal{P}}^{q-2 p^{r-j}+1}\left(I^{(r)}, S^{p^{r-j}(j)}\right)=\operatorname{Ext}_{\mathcal{P}}^{q-p^{r-j}}\left(I^{(r)}, \Lambda^{p^{r-j}(j)}\right) \rightarrow \operatorname{Ext}_{\mathcal{P}}^{q}\left(I^{(r)}, \Omega_{p^{r-j}}^{\bullet(j)}\right) \\
\rightarrow \operatorname{Ext}_{\mathcal{P}}^{q}\left(I^{(r)}, S^{p^{r-j}(j)}\right) \rightarrow 0
\end{array}
$$

and hence we have:

$$
\operatorname{Ext}_{\mathcal{P}}^{q}\left(I^{(r)}, \Omega_{p^{-j}}^{\bullet(j)}\right)= \begin{cases}k & \text { if } q \equiv 0,-1 \bmod 2 p^{r-j}, 0 \leq q<2 p^{r} \\ 0 & \text { otherwise. }\end{cases}
$$

Proof. Consider the first hypercohomology spectral sequence corresponding to the complex $\Omega_{p^{r-j}}^{\bullet(j)}$

$$
E_{1}^{s, t}=\operatorname{Ext}_{\mathcal{P}}^{t}\left(I^{(r)}, \Omega_{p^{r-j}}^{s(j)}\right) \Rightarrow \operatorname{Ext}_{\mathcal{P}}^{s+t}\left(I^{(r)}, \Omega_{p^{r-j}}^{\bullet(j)}\right) .
$$

Theorem 2.13 implies that $E_{1}^{s, t}=0$ unless $s=0$ or $p^{r-j}$. Thus the spectral sequence contains only two non zero columns: $s=0, s=p^{r-j}$. All differentials in this spectral sequence are zero exept possibly for $d_{p^{r-j}}: \operatorname{Ex} t_{\mathcal{P}}^{t}\left(I^{(r)}, S^{p^{r-j}(j)}\right) \rightarrow$ $\operatorname{Ext}_{\mathcal{P}}^{t-p^{r-j}+1}\left(I^{(r)}, \Lambda^{p^{r-j}(j)}\right)=\operatorname{Ext}_{\mathcal{P}}^{t-2 p^{r-j}+2}\left(I^{(r)}, S^{p^{r-j}(j)}\right)$. It suffices to note now that at least one of the indices of the above Ext-groups is not congruent to zero modulo $2 p^{r-j}$ and hence the corresponding group is zero according to the induction hypothesis. Vanishing of all differentials provides us with the desired exact sequences. Vanishing of one of the two end terms is clear since either $q$ or $q-2 p^{r-j}+1$ is odd.

$$
\text { (4.5.3). } \operatorname{Ext}_{\mathcal{P}}^{\text {odd }}\left(I^{(r)}, K_{p^{r-j}}^{\bullet(j)}\right)=0 \text {. }
$$

Proof. Consider the first hypercohomology spectral sequence corresponding to the complex $K_{p^{-1}}^{\bullet(j)}$ :

$$
E_{1}^{s, t}=\operatorname{Ext}_{\mathcal{P}}^{t}\left(I^{(r)}, K_{p^{r-j}}^{s(j)}\right) \Rightarrow \operatorname{Ext}_{\mathcal{P}}^{s+t}\left(I^{(r)}, K_{p^{r-j}}^{\bullet(j)}\right) .
$$

Our induction hypothesis and Proposition 4.4 show immediately that all $E_{1}$-terms of odd total degree are zero and hence the limit is also zero in odd degrees.

Consider the second hypercohomology spectral sequence corresponding to the complex $K_{p^{r-j}}^{\bullet(j)}$. Lemma 4.3 shows that it looks as follows:

$$
\left.E_{2}^{s, t}=\operatorname{Ext}_{\mathcal{P}}^{s}\left(I^{(r)}, K_{p^{r-j-1}}^{t(j+1)}\right)\right) \Rightarrow \operatorname{Ext}_{\mathcal{P}}^{s+t}\left(I^{(r)}, K_{p^{r-j}}^{\bullet(j)}\right) .
$$


(4.5.4). All differentials of the above spectral sequence coming to $E^{s, 0}$-terms are zero and hence $\left.E_{\infty}^{s, 0}=E_{2}^{s, 0}=\operatorname{Ext} t_{\mathcal{P}}^{s}\left(I^{(r)}, S^{p^{r-j-1}(j+1)}\right)\right)$.

Proof. Consider along with the spectral sequence $E$ the analogous spectral sequence $\bar{E}$ corresponding to the complex $\Omega_{p^{r-j}}^{\bullet}$. In view of Proposition 4.2 it looks as follows:

$$
\left.\bar{E}_{2}^{s, t}=\operatorname{Ext}_{\mathcal{P}}^{s}\left(I^{(r)}, \Omega_{p^{r-j-1}}^{t(j+1)}\right)\right) \Rightarrow \operatorname{Ext}_{\mathcal{P}}^{s+t}\left(I^{(r)}, \Omega_{p^{r-j}}^{\bullet(j)}\right) .
$$

The embeding of complexes $K_{p^{r-j}}^{\bullet \bullet} \hookrightarrow \Omega_{p^{r-j}}^{\bullet(j)}$ defines a homomorphism of spectral sequences $E \rightarrow \bar{E}$ and the corresponding homomorphism $E_{2}^{s, 0} \rightarrow \bar{E}_{2}^{s, 0}$ is an isomorphism. Note further that in view of the vanishing theorem 2.13 the spectral sequence $\bar{E}$ has only two nonzero rows: $t=0, t=p^{r-j-1}$ and hence all differentials $\bar{d}_{n}$ are zero except possibly for $\bar{d}_{p^{r-j-1}+1}$. Now commutativity of the diagram

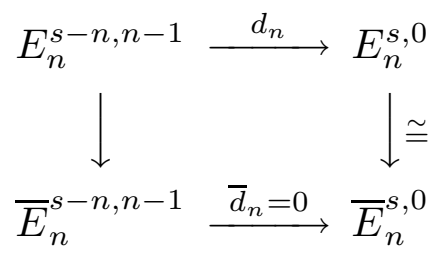

and immediate induction on $n=2, \ldots, p^{r-j-1}$ shows that for these $n$ the differential $d_{n}: E_{n}^{s-n, n-1} \rightarrow E_{n}^{s, 0}$ is trivial, $E_{n+1}^{s, 0}=E_{2}^{s, 0}$ and the map $E_{n+1}^{s, 0} \rightarrow \bar{E}_{n+1}^{s, 0}$ is an isomorphism. Finally for $n>p^{r-j-1}$ the differential $d_{n}: E_{n}^{s-n, n-1} \rightarrow E_{n}^{s, 0}$ is trivial by dimension considerations: $E^{s, t}=0$ for $t \geq p^{r-j-1}$.

(4.5.5). $\operatorname{Ext}^{\mathrm{odd}}\left(I^{(r)}, S^{p^{r-j-1}(j+1)}\right)=0$.

Proof. In view of (4.5.4) the edge homomorphism

$$
\operatorname{Ext}_{\mathcal{P}}^{*}\left(I^{(r)}, S^{p^{r-j-1}(j+1)}\right)=E_{2}^{*, 0} \rightarrow \operatorname{Ext}_{\mathcal{P}}^{*}\left(I^{(r)}, K_{p^{r-j}}^{\bullet(j)}\right)
$$

is injective. Thus our statement follows from (4.5.3).

Now we are prepared to finish the inductive step. Consider once again the second hypercohomology spectral sequence corresponding to the complex $\Omega_{p^{r-j}}^{\bullet}$ :

$$
\bar{E}_{2}^{s, t}=\operatorname{Ext}_{\mathcal{P}}^{s}\left(I(r), \Omega_{p^{r-j-1}}^{t(j+1)}\right) \Rightarrow \operatorname{Ext}_{\mathcal{P}}^{s+t}\left(I^{(r)}, \Omega_{p^{r-j}}^{\bullet(j)}\right) .
$$

As was noted in the proof of (4.5.4) this spectral sequence has only two non zero rows: $t=0$ and $t=p^{r-j-1}$ and only one nonzero differential. This, together with Proposition 4.4, gives the following exact sequences:

$$
\begin{gathered}
0 \rightarrow \bar{E}_{\infty}^{s-p^{r-j-1}-1, p^{r-j-1}} \rightarrow \bar{E}_{p^{r-j-1}}^{s-p^{r-j-1}-1, p^{r-j-1}}=\operatorname{Ext}_{\mathcal{P}}^{s-2 p^{r-j-1}}\left(I^{(r)}, S^{p^{r-j-1}(j+1)}\right) \\
\stackrel{d_{p^{r-j-1}+1}}{\longrightarrow} \operatorname{Ext}_{\mathcal{P}}^{s}\left(I^{(r)}, S^{p^{r-j-1}(j+1)}\right)=\bar{E}_{p^{r-j-1}}^{s, 0} \rightarrow \bar{E}_{\infty}^{s, 0} \rightarrow 0 \\
0 \rightarrow \bar{E}_{\infty}^{s, 0} \rightarrow \operatorname{Ext}_{\mathcal{P}}^{s}\left(I^{(r)}, \Omega_{p^{r-j}}^{\bullet(j)}\right) \rightarrow \bar{E}_{\infty}^{s-p^{r-j-1}, p^{r-j-1}} \rightarrow 0 .
\end{gathered}
$$


The first exact sequence together with (4.5.5) shows that

$$
\bar{E}_{\infty}^{s, 0}=\bar{E}_{\infty}^{s-p^{r-j-1}-1, p^{r-j-1}}=0 \text { if } s \text { is odd } .
$$

Using now the second exact sequence and (4.5.2) we come to the following computation of the $\bar{E}_{\infty}$-term:

$$
\begin{gathered}
\bar{E}_{\infty}^{s, 0}= \begin{cases}k & \text { if } s \equiv 0 \bmod 2 p^{r-j}, 0 \leq s<2 p^{r} \\
0 & \text { otherwise }\end{cases} \\
\bar{E}_{\infty}^{s-p^{r-j-1}-1, p^{r-j-1}}= \begin{cases}k & \text { if } s \equiv 0 \bmod 2 p^{r-j}, 0<s \leq 2 p^{r} \\
0 & \text { otherwise. }\end{cases}
\end{gathered}
$$

Using these formulae and the first of the above exact sequences in an iterative argument, we easily conclude the computation.

We record for the future use additional information that we obtain in this argument.

(4.5.6).

(1) Assume that $s \not \equiv 0 \bmod 2 p^{r-j}$. Then the differential

$$
\bar{d}_{p^{r-j-1}+1}: \operatorname{Ext}_{\mathcal{P}}^{s-2 p^{r-j-1}}\left(I^{(r)}, S^{p^{r-j-1}(j+1)}\right) \rightarrow \operatorname{Ext}_{\mathcal{P}}^{s}\left(I^{(r)}, S^{p^{r-j-1}(j+1)}\right)
$$

is an isomorphism. In particular, $\operatorname{Ext}_{\mathcal{P}}^{s}\left(I^{(r)}, S^{p^{r-j-1}(j+1)}\right)=0$ if $s \not \equiv 0 \bmod 2 p^{r-j-1}$.

(2) Assume that $s \equiv 0 \bmod 2 p^{r-j}$. Then the natural homomorphism

$$
\operatorname{Ext}_{\mathcal{P}}^{s}\left(I^{(r)}, S^{p^{r-j-1}(j+1)}\right) \rightarrow \operatorname{Ext}_{\mathcal{P}}^{s}\left(I^{(r)}, S^{p^{r-j}(j)}\right)
$$

induced by the embeding $S^{p^{r-j-1}(j+1)} \hookrightarrow S^{p^{r-j}(j)}$ is an isomorphism and the differential

$$
\bar{d}_{p^{r-j-1}+1}: \operatorname{Ext}_{\mathcal{P}}^{s-2 p^{r-j-1}}\left(I^{(r)}, S^{p^{r-j-1}(j+1)}\right) \rightarrow \operatorname{Ext}_{\mathcal{P}}^{s}\left(I^{(r)}, S^{p^{r-j-1}(j+1)}\right)
$$

is trivial.

(3) $\left(\bar{d}_{p^{r-j-1}+1}\right)^{p}: \operatorname{Ext}_{\mathcal{P}}^{s-2 p^{r-j}}\left(I^{(r)}, S^{p^{r-j-1}(j+1)}\right) \rightarrow \operatorname{Ext}_{\mathcal{P}}^{s}\left(I^{(r)}, S^{p^{r-j-1}(j+1)}\right)$ is the zero map

The following statement is clear from (4.5.6).

Corollary 4.6. Assume that $0 \leq j<r$. Let $V \subset \operatorname{Ext}_{\mathcal{P}}^{*}\left(I^{(r)}, S^{p^{r-j-1}(j+1)}\right)$ be a graded subspace such that

(1) $V$ maps surjectively onto $\operatorname{Ext}_{\mathcal{P}}^{*}\left(I^{(r)}, S^{p^{r-j}(j)}\right)$.

(2) $V$ is stable with respect to the action of the endomorphism

$$
\bar{d}_{p^{r-j-1}+1}: \operatorname{Ext}_{\mathcal{P}}^{*-2 p^{r-j-1}}\left(I^{(r)}, S^{p^{r-j-1}(j+1)}\right) \rightarrow \operatorname{Ext}_{\mathcal{P}}^{*}\left(I^{(r)}, S^{p^{r-j-1}(j+1)}\right) .
$$


Then $V=\operatorname{Ext}_{\mathcal{P}}^{*}\left(I^{(r)}, S^{p^{r-j-1}(j+1)}\right)$.

Theorem 4.5 shows that the $k$-vector space $\operatorname{Ext}_{\mathcal{P}}^{2 p^{r-1}}\left(I^{(r)}, I^{(r)}\right)$ is one dimensional. We define $e_{r} \in \operatorname{Ext}_{\mathcal{P}}^{2 p^{r-1}}\left(I^{(r)}, I^{(r)}\right)$ to be a distinguished basis element of this vector space. The particular choice of $e_{r}$ is given by the following procedure. Denote by $\phi_{r} \in \operatorname{Hom}_{\mathcal{P}}\left(I^{(r)}, S^{p^{r}}\right)$ the standard embeding : $v^{(r)} \mapsto v^{p^{r}}$. Take $j=0$ and consider once again the above spectral sequence $\bar{E}$. According to (4.5.6) $\bar{e}_{r}=\bar{d}_{p^{r-1}+1}\left(\phi_{r-1}^{(1)}\right)$ is a basis element of $\operatorname{Ext}_{\mathcal{P}}^{2 p^{r-1}}\left(I^{(r)}, S^{p^{r-1}(1)}\right)$. Furthermore (4.5.6) shows that the sequence of homomorphisms

$$
\operatorname{Ext}_{\mathcal{P}}^{2 p^{r-1}}\left(I^{(r)}, I^{(r)}\right) \rightarrow \operatorname{Ext}_{\mathcal{P}}^{2 p^{r-1}}\left(I^{(r)}, S^{p(r-1)}\right) \rightarrow \ldots \rightarrow \operatorname{Ext}_{\mathcal{P}}^{2 p^{r-1}}\left(I^{(r)}, S^{p^{r-1}(1)}\right)
$$

consists of isomorphisms. We define $e_{r}$ to be the inverse image of $\bar{e}_{r}$ in $\operatorname{Ext}_{\mathcal{P}}^{2 p^{r-1}}\left(I^{(r)}, I^{(r)}\right)$, so that the following equality holds:

$$
\phi_{r-1}^{(1)} \cdot e_{r}=\bar{e}_{r}=\bar{d}_{p^{r-1}+1}\left(\phi_{r-1}^{(1)}\right) .
$$

Now we can obtain, following [FLS], the multiplicative structure of the Yoneda algebra $\operatorname{Ext}_{\mathcal{P}}^{*}\left(I^{(r)}, I^{(r)}\right)$. We are going to show that this is a commutative $k$-algebra spanned by the generators $e_{r} \in \operatorname{Ext}_{\mathcal{P}}^{2 p^{r-1}}\left(I^{(r)}, I^{(r)}\right), e_{r-1}^{(1)} \in \operatorname{Ext}_{\mathcal{P}}^{2 p^{r-2}}\left(I^{(r)}, I^{(r)}\right), \ldots$, $e_{1}^{(r-1)} \in \operatorname{Ext}_{\mathcal{P}}^{2}\left(I^{(r)}, I^{(r)}\right)$ subject only to the following relations : $e_{r}^{p}=0, \ldots$, $\left(e_{1}^{(r-1)}\right)^{p}=0$.

Note that for any complex $C^{\bullet}$ (of degree +1$) \operatorname{Ex} t_{\mathcal{P}}^{*}\left(I^{(r)}, C^{\bullet}\right.$ ) is naturally a right module over the Yoneda algebra $\operatorname{Ext}_{\mathcal{P}}^{*}\left(I^{(r)}, I^{(r)}\right)$. Moreover both hypercohomology spectral sequences converging to $\operatorname{Ext}_{\mathcal{P}}^{*}\left(I^{(r)}, C^{\bullet}\right)$ admit a natural right action of $\operatorname{Ext}_{\mathcal{P}}^{*}\left(I^{(r)}, I^{(r)}\right)$.

Corollary 4.7. For any $j, 0 \leq j \leq r$ a basis of the graded vector space $\operatorname{Ext}_{\mathcal{P}}^{*}\left(I^{(r)}, S^{p^{r-j}(j)}\right)$ consists of products of the form

$$
\phi_{r-j}^{(j)} \cdot\left(e_{r-j+1}^{(j-1)}\right)^{l_{1}} \cdot \ldots \cdot\left(e_{r}\right)^{l_{j}}
$$

$\left(0 \leq l_{1}, \ldots, l_{j}<p\right)$. Moreover the action of the endomorphism $\bar{d}_{p^{r-j}+1}$ : $\operatorname{Ext}_{\mathcal{P}}^{s-2 p^{r-j}}\left(I^{(r)}, S^{p^{r-j}(j)}\right) \rightarrow \operatorname{Ext}_{\mathcal{P}}^{s}\left(I^{(r)}, S^{p^{r-j}(j)}\right)$ takes $\phi_{r-j}^{(j)} \cdot\left(e_{r-j+1}^{(j-1)}\right)^{l_{1}} \cdot \ldots \cdot\left(e_{r}\right)^{l_{j}}$ to $\phi_{r-j}^{(j)} \cdot\left(e_{r-j+1}^{(j-1)}\right)^{l_{1}+1} \cdot \ldots \cdot\left(e_{r}\right)^{l_{j}}$ if $l_{1}<p$ and to zero otherwise.

Proof. We proceed by induction on $j$. The case $j=0$ is trivial. Assuming that the result is true for $j<r$ we proceed to show that it holds for $j+1$ as well. To simplify notations we will drop the indices of all differentials $\bar{d}$ that we are going to consider. Denote by $V$ the vector subspace of $\operatorname{Ext}_{\mathcal{P}}^{*}\left(I^{(r)}, S^{p^{r-j-1}(j+1)}\right)$ spanned by the above products. To show that $V$ coincides with $\operatorname{Ext}_{\mathcal{P}}^{*}\left(I^{(r)}, S^{p^{r-j-1}(j+1)}\right)$ it suffices in view of Corollary 4.6 to check that $V$ maps surjectively onto $\operatorname{Ext}_{\mathcal{P}}^{*}\left(I^{(r)}, S^{p^{r-j}(j)}\right)$ and is stable with respect to the action of $\bar{d}$. The first fact is immediate from the induction hypothesis since the image of $\phi_{r-j-1}^{(j+1)} \in \operatorname{Hom}_{\mathcal{P}}\left(I^{(r)}, S^{p^{r-j-1}(j+1)}\right)$ in 
$\operatorname{Hom}_{\mathcal{P}}\left(I^{(r)}, S^{p^{r-j}(j)}\right)$ is equal to $\phi_{r-j}^{(j)}$. To prove the second fact, we start with the following observation. The element $e_{r-j}$ was defined from the following equation

$$
\phi_{r-j-1}^{(1)} \cdot e_{r-j}=\bar{e}_{r-j}=d\left(\phi_{r-j-1}^{(1)}\right) \in \operatorname{Ext}_{\mathcal{P}}^{2 p^{r-j-2}}\left(I^{(r-j-1)}, S^{p^{r-j-2}(1)}\right)
$$

where $d=d_{p^{r-j}+1}$ is the differential in the spectral sequence

$$
E_{2}^{s, t}=\operatorname{Ext}_{\mathcal{P}}^{s}\left(I^{(r-j)}, \Omega_{p^{r-j-1}}^{t(1)}\right) \Rightarrow \operatorname{Ext}_{\mathcal{P}}^{s+t}\left(I^{(r-j)}, \Omega_{p^{r-j}}^{\bullet}\right)
$$

Keep the notation $\bar{E}$ for the corresponding spectral sequence defined by the complex $\Omega_{p^{r-j}}^{\bullet(j)}$

$$
\bar{E}_{2}^{s, t}=\operatorname{Ext}_{\mathcal{P}}^{s}\left(I^{(r)}, \Omega_{p^{r-j-1}}^{t(j+1)}\right) \Rightarrow \operatorname{Ext}_{\mathcal{P}}^{s+t}\left(I^{(r)}, \Omega_{p^{r-j}}^{\bullet(j)}\right) .
$$

Since the twisting functor is exact it induces a homomorphism of spectral sequences $E \rightarrow \bar{E}$ compatible with the twisting homomorphism of algebras $\operatorname{Ext}_{\mathcal{P}}^{*}\left(I^{(r-j)}, I^{(r-j)}\right) \rightarrow \operatorname{Ext}_{\mathcal{P}}^{*}\left(I^{(r)}, I^{(r)}\right)$. Moreover on $E_{2}$-terms this homomorphism clearly coincides with the twisting homomorphism $\lambda \mapsto \lambda^{(j)}$ introduced previously. The net result of this general nonsense discussion is that by twisting the equation (4.7.1) we get the formula $\phi_{r-j-1}^{(j+1)} \cdot e_{r-j}^{(j)}=\bar{d}\left(\phi_{r-j-1}^{(j+1)}\right)$. This formula shows immediately that $\bar{d}$ takes $\phi_{r-j-1}^{(j+1)} \cdot\left(e_{r-j}^{(j)}\right)^{l_{1}} \cdot \ldots \cdot\left(e_{r}\right)^{l_{j+1}}$ to $\phi_{r-j-1}^{(j+1)} \cdot\left(e_{r-j}^{(j)}\right)^{l_{1}+1} \cdot \ldots \cdot\left(e_{r}\right)^{l_{j+1}}$. Furthermore the formula $\bar{d}^{p}=0$ shows that $\phi_{r-j-1}^{(j+1)} \cdot\left(e_{r-j}^{(j)}\right)^{p}=0$. This concludes the proof that $V$ is stable with respect to the action of $\bar{d}$ and hence coincides with $\operatorname{Ext}_{\mathcal{P}}^{*}\left(I^{(r)}, S^{p^{r-j-1}(j+1)}\right)$. The fact that the products $\phi_{r-j-1}^{(j+1)} \cdot\left(e_{r-j}^{(j)}\right)^{l_{1}} \cdot \ldots$. $\left(e_{r}\right)^{l_{j+1}} \quad\left(0 \leq l_{i}<p\right)$ are linearly independent follows now by dimension counting.

Corollary 4.8. The basis of the Yoneda algebra Ext $\operatorname{P}_{\mathcal{P}}^{*}\left(I^{(r)}, I^{(r)}\right)$ consists of products of the form $\left(e_{1}^{(r-1)}\right)^{l_{1}} \cdot \ldots \cdot e_{r}^{l_{r}} \quad\left(0 \leq l_{1}, \ldots, l_{r}<p\right)$. For every $i(1 \leq i \leq r)$ we have the relation $\left(e_{i}^{(r-i)}\right)^{p}=0$.

Proof. Corollary 4.7 applied to $j=r$ gives the first statement. The element $e_{i}^{p}$ lies in the trivial group $\operatorname{Ext}_{\mathcal{P}}^{2 p^{i}}\left(I^{(i)}, I^{(i)}\right)$ and hence is equal to zero. Twisting $r-i$ times the equation $e_{i}^{p}=0$ we get the desired relation.

Corollary 4.9. The twisting homomorphism

$$
\operatorname{Ext}_{\mathcal{P}}^{*}\left(I^{(r)}, I^{(r)}\right) \rightarrow \operatorname{Ext}_{\mathcal{P}}^{*}\left(I^{(r+1)}, I^{(r+1)}\right)
$$

is injective.

Theorem 4.10. The Yoneda algebra $E(r, k)=\operatorname{Ext}_{\mathcal{P}}^{*}\left(I^{(r)}, I^{(r)}\right)$ is a commutative $k$-algebra generated by elements $e_{r}, \ldots, e_{1}^{(r-1)}$ subject only to relations $e_{r}^{p}=\ldots=$ $\left(e_{1}^{(r-1)}\right)^{p}=0$.

Proof. In view of Corollary 4.8, it suffices to check the commutativity of $E(r, k)$. Since the natural homomorphism $E\left(r, \mathbb{F}_{p}\right) \otimes_{\mathbb{F}_{p}} k \rightarrow E(r, k)$ is an isomorphism, we may assume further that $k=\mathbb{F}_{p}$. Note that in this case the polynomial functor $I^{(1)}$ is identity on objects, whereas for any $V, W \in \mathcal{V}$ the polynomial map 
$I_{V, W}^{(1)} \in \operatorname{Hom}_{P o l}\left(\operatorname{Hom}_{\mathbb{F}_{p}}(V, W), \operatorname{Hom}_{\mathbb{F}_{p}}(V, W)\right)=\operatorname{Hom}_{\mathbb{F}_{p}}\left(H_{o m} \mathbb{F}_{p}(V, W)^{\#}\right.$, $\left.S^{*}\left(\operatorname{Hom}_{\mathbb{F}_{p}}(V, W)^{\#}\right)\right)$ coincides with raising to the $p$-th power

$$
H o m_{\mathbb{F}_{p}}(V, W)^{\#} \rightarrow S^{p}\left(H o m_{\mathbb{F}_{p}}(V, W)^{\#}\right), \quad\left(f \mapsto f^{p}\right) .
$$

In other words $I_{V, W}^{(1)}$ may be identified with the Frobenius morphism on the scheme $\operatorname{Hom}_{\mathbb{F}_{p}}(V, W)\left(=\operatorname{Spec} S^{*}\left(\operatorname{Hom}_{\mathbb{F}_{p}}(V, W)^{\#}\right)\right.$. Since the Frobenius morphism is functorial on the category of $\mathbb{F}_{p}$-schemes we conclude that for any polynomial functor $T \in \mathcal{P}$ the following diagram of polynomial maps commute

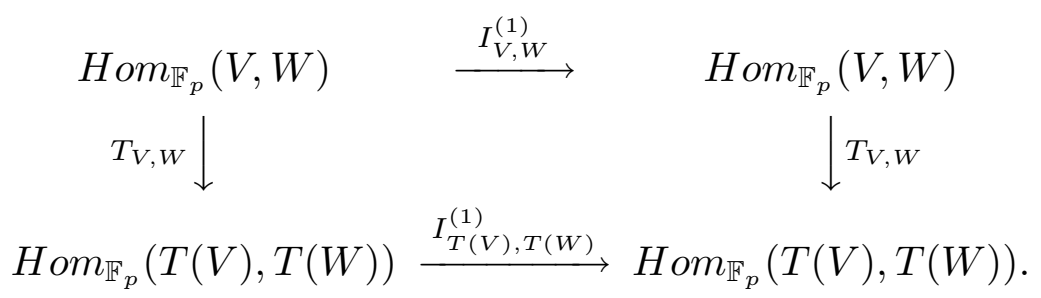

This shows that the polynomial functors $I^{(1)} \circ T$ and $T \circ I^{(1)}=T^{(1)}$ coincide. Assume now that we are given two polynomial functors $T, T^{\prime} \in \mathcal{P}_{d}$ and elements $e \in \operatorname{Ext}_{\mathcal{P}}^{n}\left(I^{(r)}, I^{(r)}\right), e^{\prime} \in \operatorname{Ext}_{\mathcal{P}}^{m}\left(T, T^{\prime}\right)$. The exact functor $\mathcal{P} \rightarrow \mathcal{P} \quad(U \mapsto$ $U \circ T)$ induces a homomorphism $\operatorname{Ext}_{\mathcal{P}}^{*}\left(I^{(r)}, I^{(r)}\right) \rightarrow \operatorname{Ext}_{\mathcal{P}}^{*}\left(I^{(r)} \circ T, I^{(r)} \circ T\right)=$ $\operatorname{Ext}_{\mathcal{P}}^{*}\left(T^{(r)}, T^{(r)}\right)$. We will denote the image of $e$ under this homomorphism by $e(T)$.

Lemma 4.10 .1 (cf. $[\mathrm{FLS}]) . e^{\prime(r)} \cdot e(T)=(-1)^{n m} e\left(T^{\prime}\right) \cdot e^{\prime(r)} \in \operatorname{Ext}_{\mathcal{P}}^{n+m}\left(T^{(r)}, T^{\prime(r)}\right)$.

Proof. The general case of the Lemma is easily reduced to the case $m=1$. Furthermore we may clearly assume that $d>0$. Assume that $e$ and $e^{\prime}$ are represented by extensions of polynomial functors

$$
\begin{aligned}
0 \rightarrow I^{(r)} & \rightarrow U^{1} \rightarrow \ldots \rightarrow U^{n} \rightarrow I^{(r)} \rightarrow 0 \\
0 & \rightarrow T^{\prime} \rightarrow S \rightarrow T \rightarrow 0
\end{aligned}
$$

Consider the commutative diagram with exact rows

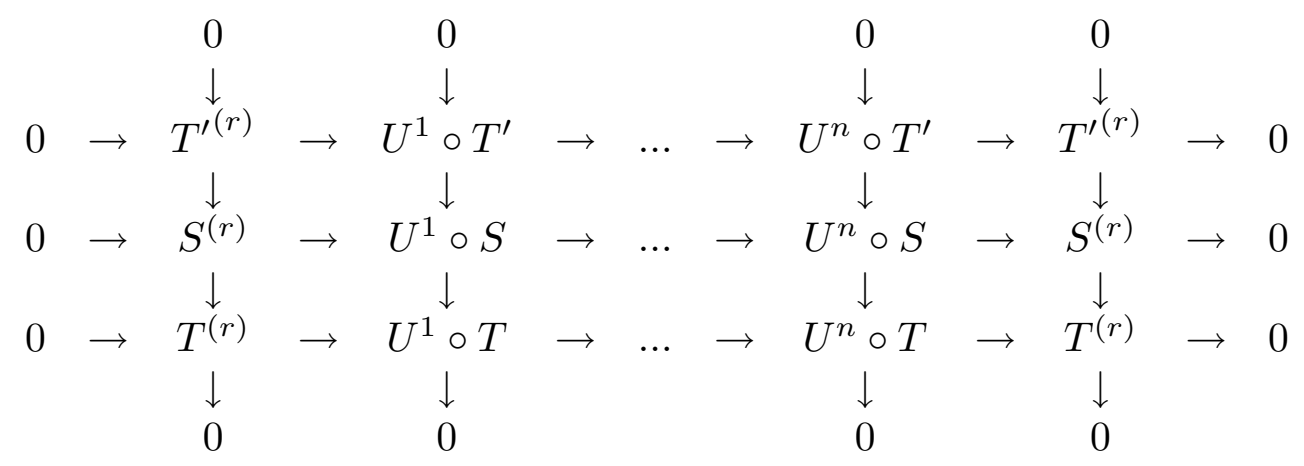

The first and the last column of this diagram are exact. All the other columns are at least complexes (since $U^{i}(0)=0$ ). It's well known (see[FLS]) and easy to prove that whenever we have a diagram of such kind the extension obtained by composing 
the last column with the top row differs by a sign $(-1)^{n}$ from the extension obtained by composing the bottom row with the first column.

Now we can conclude the proof of the Theorem 4.10. Let $e, e^{\prime} \in E\left(r, \mathbb{F}_{p}\right)$ be two homogenous elements of degrees $n$ and $m$ respectively $(n \equiv m \equiv 0 \bmod 2)$. Applying (4.10.1) to $T=T^{\prime}=I^{(r)}$ we get the following equation: $\left(e \cdot e^{\prime}\right)^{(r)}=$ $e^{(r)} \cdot e^{\prime(r)}=e\left(I^{(r)}\right) \cdot e^{\prime(r)}=e^{\prime(r)} \cdot e\left(I^{(r)}\right)=e^{\prime(r \cdot)} e^{(r)}=\left(e^{\prime} \cdot e\right)^{(r)}$. Since the twisting homomorphism is injective this equation implies that $e \cdot e^{\prime}=e^{\prime} \cdot e$.

Let $n \geq 0$ be an integer. We will use the same notation $e_{r}$ for the image of $e_{r} \in \operatorname{Ext}_{\mathcal{P}}^{2 p^{r-1}}\left(I^{(r)}, I^{(r)}\right)$ in $\operatorname{Ext}_{G L_{n, k}}^{2 p^{r-1}}\left(\left(k^{n}\right)^{(r)},\left(k^{n}\right)^{(r)}\right)=H^{2 p^{r-1}}\left(G L_{n, k}, g l_{n}^{(r)}\right)$. The following result is a combination of (4.8) and (3.13).

Corollary 4.11. Assume that $n \geq p^{r}$. Then a basis of the Yoneda algebra $H^{*}\left(G L_{n, k}, g l_{n}^{(r)}\right)=\operatorname{Ext}_{G L_{n, k}}\left(\left(k^{n}\right)^{(r)},\left(k^{n}\right)^{(r)}\right)$ consists of products of the form $\left(e_{1}^{(r-1)}\right)^{l_{0}} \cdot \ldots \cdot e_{r}^{l_{r-1}} \quad\left(0 \leq l_{0}, \ldots, l_{r-1}<p\right)$. In other words $H^{m}\left(G L_{n, k}, g l_{n}^{(r)}\right)=0$ if $m$ is odd or $m \geq 2 p^{r}$. If $m=2 l<2 p^{r}$ is even and $l=l_{0}+l_{1} p+\ldots+l_{r-1} p^{r-1} \quad(0 \leq$ $\left.l_{0}, \ldots, l_{r-1}<p\right)$ is the $p$-adic expansion of $l$, then $H^{m}\left(G L_{n, k}, g l_{n}^{(r)}\right)$ is a one dimensional $k$-vector space with basis $\left(e_{1}^{(r-1)}\right)^{l_{0}} \cdot \ldots \cdot e_{r}^{l_{r-1}}$.

Finally we identify the element $e_{1} \in \operatorname{Ext}_{\mathcal{P}}^{2}\left(I^{(1)}, I^{(1)}\right)$.

Lemma 4.12. The element $e_{1}$ is represented by the extension

$$
E_{1}: \quad 0 \rightarrow I^{(1)} \stackrel{\phi_{1}}{\longrightarrow} S^{p} \stackrel{\alpha}{\longrightarrow} \Gamma^{p} \stackrel{\phi_{1}^{\#}}{\longrightarrow} I^{(1)} \rightarrow 0
$$

where $\alpha_{V}: S^{p}(V) \rightarrow \Gamma^{p}(V)$ is the symmetrization homomorphism:

$$
\alpha_{V}\left(v_{1} \cdot \ldots \cdot v_{p}\right)=\sum_{\sigma \in \Sigma_{p}} v_{\sigma^{-1}(1)} \otimes \ldots \otimes v_{\sigma^{-1}(p)}
$$

Proof. It is easy to verify that the above sequence is exact. The class $e_{1}$ was defined via the formula $e_{1}=\bar{d}_{2}\left(1_{I^{(1)}}\right)$, where $\bar{d}_{2}$ is the differential in the second hypercohomology spectral sequence corresponding to the complex $\Omega_{p}^{\bullet}$. According to the theorem of Cartier, the complex $\Omega_{p}^{\bullet}$ has only two nonzero homology groups: $H^{0}\left(\Omega_{p}^{\bullet}\right)=H^{1}\left(\Omega_{p}^{\bullet}\right)=I^{(1)}$. For any complex any two adjacent homology groups are related by means of a 2 -fold extension and the differential $\bar{d}_{2}$ coincides with left multiplication by the class of this extension. In the present case this extension looks as follows.

$$
0 \rightarrow I^{(1)} \rightarrow \Omega_{p}^{0} \rightarrow Z_{p}^{1} \rightarrow I^{(1)} \rightarrow 0
$$

Thus we only have to compare $E_{1}$ with the above extension. To do so we consider the composition

$$
\Gamma^{p} \rightarrow \Gamma^{p-1} \otimes I \stackrel{\sim}{\longrightarrow} S^{p-1} \otimes I=S^{p-1} \otimes \Lambda^{1}=\Omega_{p}^{1}
$$


Here the first arrow is comultiplication and the second one is induced by the natural isomorphism $\Gamma^{p-1} \stackrel{\sim}{\longrightarrow} S^{p-1}$. One checks easily that this homomorphism takes $\Gamma^{p}$ to $Z_{p}^{1} \subset \Omega_{p}^{1}$ and that the following diagram commutes

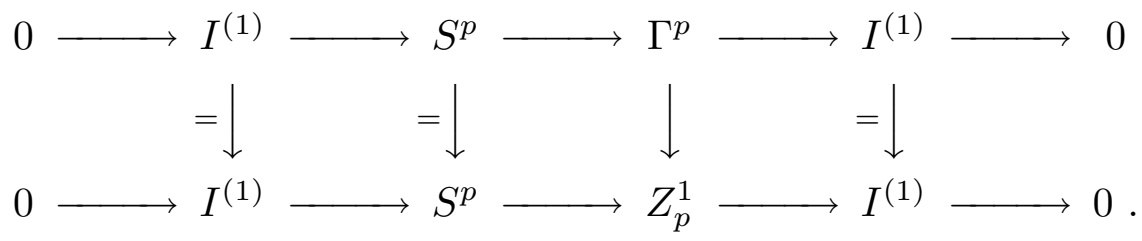

\section{$\S 5$ The Compatibility Diagram}

Having constructed the universal class $e_{r} \in \operatorname{Ext}_{\mathcal{P}}^{2 p^{r-1}}\left(I^{(r)}, I^{(r)}\right)$ for each $r \geq 1$, we now proceed to relate this class to the $p^{r-1}$-st tensor power of $e_{1}$. This will permit us in the next section to verify that $e_{r}$ has non-trivial restriction in the cohomology of $\left(G L_{n}\right)_{(1)}(n \geq 2)$. In fact, as the reader will see, our methods require us to consider the $p^{r-1}$-st tensor power of $e_{1}^{p-1}$ and relate this to $e_{r}^{p-1}$.

Let $S, T, \widetilde{S}, \widetilde{T}$ be polynomial functors. Consider projective resolutions $P_{\bullet} \rightarrow$ $S, \widetilde{P} \bullet \sim \widetilde{S}$. Corollary 2.11 shows that the complex $P \bullet \otimes \widetilde{P} \bullet$ is a projective resolution of $S \otimes \widetilde{S}$. Thus the map on the cohomology induced by the natural homomorphism of complexes

$$
\operatorname{Hom}_{\mathcal{P}}\left(P_{\bullet}, T\right) \otimes \operatorname{Hom}_{\mathcal{P}}(\tilde{P} \bullet, \tilde{T}) \rightarrow H_{\mathcal{P}}\left(P_{\bullet} \otimes \widetilde{P} \bullet, T \otimes \tilde{T}\right)
$$

takes the form

$$
\bigoplus_{i+j=n} \operatorname{Ext}_{\mathcal{P}}^{i}(S, T) \otimes \operatorname{Ext}_{\mathcal{P}}^{j}(\tilde{S}, \tilde{T}) \rightarrow \operatorname{Ext}_{\mathcal{P}}^{n}(S \otimes \tilde{S}, T \otimes \tilde{T}) .
$$

The corresponding map $\operatorname{Ext}_{\mathcal{P}}^{i}(S, T) \otimes \operatorname{Ext}_{\mathcal{P}}^{j}(\tilde{S}, \widetilde{T}) \rightarrow \operatorname{Ext}_{\mathcal{P}}^{i+j}(S \otimes \widetilde{S}, T \otimes \widetilde{T})$ is called the external or tensor product operation.

Our main objective in this section is the investigation of the maps in the following commutative diagram of Ext-groups

\section{Diagram 5.1.}

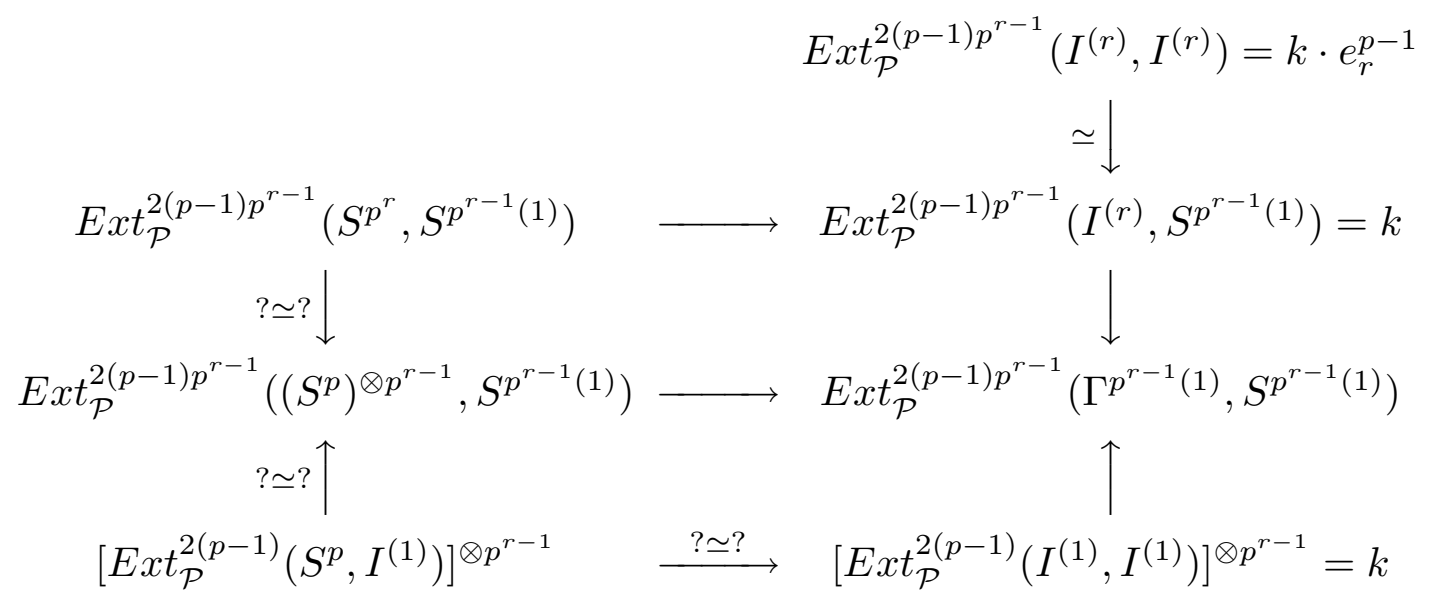


Here the bottom vertical arrows are defined via the tensor product operation and natural homomorphisms $\left(I^{(1)}\right)^{\otimes p^{r-1}} \rightarrow S^{p^{r-1}}\left(I^{(1)}\right)=S^{p^{r-1}(1)}, \Gamma^{p^{r-1}(1)}=$ $\Gamma^{p^{r-1}}\left(I^{(1)}\right) \hookrightarrow\left(I^{(1)}\right)^{\otimes p^{r-1}}$. All the other maps are induced by functor homomorphisms such as

$$
I^{(s)} \rightarrow S^{p^{s}} \leftarrow I^{\otimes p^{s}} \leftarrow \Gamma^{p^{s}} \rightarrow I^{(s)}
$$

and their twists. The indicated computation of the first, second, and fourth Extgroups in the right column of the diagram is done in Theorem 4.5 and Corollary 4.8. Moreover (4.5.6) provides the upper isomorphism.

We proceed to prove in the following series of propositions that the two left vertical maps and the bottom horizontal map (decorated with ? $\simeq$ ?) are also isomorphisms.

The following proposition verifies that the lower left vertical arrow of Diagram 5.1 is an isomorphism (by taking $j=1$ and considering the $p^{r-1}$-fold tensor product $\left.\left(S^{p}\right)^{\otimes p^{r-1}}\right)$.

Proposition 5.2. Let $T$ and $T^{\prime}$ be homogenous polynomial functors of degrees $d$ and $d^{\prime}$ respectively $\left(d+d^{\prime}=s \cdot p^{j}\right.$ for some $\left.j \geq 1\right)$. Then

(1) $\operatorname{Ext}_{\mathcal{P}}^{*}\left(T \otimes T^{\prime}, S^{s(j)}\right)=0$ if $d \not \equiv 0 \bmod p^{j}$.

(2) If $d=p^{j} \cdot m, d^{\prime}=p^{j} \cdot m^{\prime}$ (so that $m+m^{\prime}=s$ ), then the canonical homomorphism $S^{m(j)} \otimes S^{m^{\prime}(j)} \rightarrow S^{s(j)}$ and the tensor product operation induce an isomorphism $\operatorname{Ext}_{\mathcal{P}}^{*}\left(T, S^{m(j)}\right) \otimes \operatorname{Ext}_{\mathcal{P}}^{*}\left(T^{\prime}, S^{m^{\prime}(j)}\right) \rightarrow \operatorname{Ext}_{\mathcal{P}}^{*}(T \otimes$ $\left.T^{\prime}, S^{s(j)}\right)$.

Proof. We first consider the special case in which $T, T^{\prime}$ are tensor products of divided power functors so that $T \otimes T^{\prime}$ is again of this form and we may thus apply Corollary 2.12. Since $T, T^{\prime}$ and $T \otimes T^{\prime}$ are projective in this case, we need only check assertions (1) and (2) for Ext $t^{0}=$ Hom. Write $T=\Gamma^{d_{1}} \otimes \cdots \otimes \Gamma^{d_{u}}, T^{\prime}=$ $\Gamma^{d_{1}^{\prime}} \otimes \cdots \otimes \Gamma^{d_{v}^{\prime}}$, so that in the notation of Corollary 2.12

$$
\operatorname{Hom}_{\mathcal{P}}\left(T \otimes T^{\prime}, S^{s(j)}\right)=\left(S^{s}\left(\left(k^{u+v}\right)^{(j)}\right)\right)^{d_{1}, \ldots, d_{u}, d_{1}^{\prime}, \ldots, d_{v}^{\prime}} .
$$

Since all the weights of the algebraic group $\mathbf{G}_{m}^{\times u+v}$ acting on $\left(k^{u+v}\right)^{(j)}=\left(k^{(j)}\right)^{u+v}$ are divisible by $p^{j}$, all the weights appearing in $S^{s}\left(\left(k^{u+v}\right)^{(j)}\right)$ are also divisible by $p^{j}$. In particular, the above Hom-group is zero if at least one of $d_{i}$ or $d_{t}^{\prime}$ is not divisible by $p^{j}$.

Assume now that $d_{i}=p^{j} \cdot m_{i}$ and $d_{t}^{\prime}=p^{j} \cdot m_{t}^{\prime}$ for all $i, 1 \leq i \leq u$ and $t, 1 \leq t \leq$ $v$. Observe that the only homogeneous polynomials of multi-degree $d_{1}, \ldots, d_{n}$ in $k\left[x_{1}, \ldots, x_{n}\right]_{d}=S^{d}\left(k^{n}\right)$ are scalar multiples of $x_{1}^{d_{1}} \cdots x_{n}^{d_{n}}$; hence

$$
\begin{aligned}
& \left(S^{s}\left(\left(k^{u+v}\right)^{(j)}\right)\right)^{d_{1}, \ldots, d_{u}, d_{1}^{\prime}, \ldots, d_{v}^{\prime}}= \\
& =S^{m_{1}}\left(k^{(j)}\right) \otimes \ldots \otimes S^{m_{u}}\left(k^{(j)}\right) \otimes S^{m_{1}^{\prime}}\left(k^{(j)}\right) \otimes \ldots \otimes S^{m_{v}^{\prime}}\left(k^{(j)}\right) .
\end{aligned}
$$

On the other hand, this tensor product (of 1 dimensional vector spaces) equals

$$
\begin{aligned}
\left(S^{m}\left(\left(k^{u}\right)^{(j)}\right)\right)^{d_{1}, \ldots, d_{u}} & \otimes\left(S^{m^{\prime}}\left(\left(k^{v}\right)^{(j)}\right)\right)^{d_{1}^{\prime}, \ldots, d_{v}^{\prime}}= \\
& =H_{\mathcal{O}}\left(T, S^{m(j)}\right) \otimes \operatorname{Hom}_{\mathcal{P}}\left(T^{\prime}, S^{m^{\prime}(j)}\right) .
\end{aligned}
$$


For general $T$ and $T^{\prime}$, we employ projective resolutions consisting of direct sums of objects of the above type. Taking the tensor product of these resolutions we get a projective resolution of the functor $T \otimes T^{\prime}$. Applying $\operatorname{Hom}_{\mathcal{F}}\left(-, S^{s(j)}\right)$ to this resolution and using the previous computations, we get the desired result.

Remark 5.3. We can obtain another proof of Theorem 2.13 in the special case $A=$ $I^{(j)}$ by considering the dual version of Proposition 5.2. One obtains an assertion for $\operatorname{Ext}_{\mathcal{P}}^{*}\left(\Gamma^{s(j)}, T \otimes T^{\prime}\right)$ which for $s=1$ is the vanishing statement of Theorem 2.13 with $A=I^{(j)}$.

The role of the $(p-1)$-st power becomes evident in the next proposition: we obtain non-zero extension classes relating symmetric functors in degrees which are multiples of $2(p-1)$ (provided $j$ in the following proposition is taken to equal 1 ).

\section{Proposition 5.4.}

$$
\begin{gathered}
\operatorname{Ext}_{\mathcal{P}}^{n}\left(\Lambda^{a p^{j}}, S^{a(j)}\right)= \begin{cases}k & a=1, n=p^{j}-1 \text { or } a=0, n=0 \\
0 & \text { otherwise }\end{cases} \\
\operatorname{Ext}_{\mathcal{P}}^{n}\left(S^{a p^{j}}, S^{a(j)}\right)= \begin{cases}k & n=2 a\left(p^{j}-1\right) \\
0 & \text { otherwise. }\end{cases}
\end{gathered}
$$

Proof. Dualizing the Koszul complex $K o_{n}^{\bullet}$, we obtain the following exact sequence of functors

$$
K o_{n}^{\#}: \quad 0 \rightarrow \Gamma^{n} \rightarrow \Gamma^{n-1} \otimes \Lambda^{1} \rightarrow \cdots \rightarrow \Gamma^{1} \otimes \Lambda^{n-1} \rightarrow \Lambda^{n} \rightarrow 0 .
$$

Consider the first hypercohomology spectral sequence associated to the acyclic complex $K o_{p^{j}}^{\#}$ :

$$
E_{1}^{s, t}=\operatorname{Ext}_{\mathcal{P}}^{t}\left(\Gamma^{s} \otimes \Lambda^{p^{j}-s}, I^{(j)}\right) \Longrightarrow 0 .
$$

By Proposition 5.2, $E_{1}^{s, t}=0$ unless $s=0$ or $s=p^{j}$. Thus, the differential

$$
d_{p^{j}}: \operatorname{Ext}_{\mathcal{P}}^{t}\left(\Lambda^{p^{j}}, I^{(j)}\right) \rightarrow \operatorname{Ext}^{t-\left(p^{j}-1\right)}\left(\Gamma^{p^{j}}, I^{(j)}\right)
$$

is an isomorphism. Since

$$
\operatorname{Hom}_{\mathcal{P}}\left(\Gamma^{p^{j}}, I^{(j)}\right)=I^{(j)}(k)=k, \quad \operatorname{Ext}_{\mathcal{P}}^{t}\left(\Gamma^{p^{k}},-\right)=0, t>0,
$$

the first computation is valid for $a=1$ (and certainly for $a=0$ as well).

Assume now that $a>1$ and consider the spectral sequence

$$
E_{1}^{s, t}=\operatorname{Ext} t_{\mathcal{P}}^{t}\left(\Gamma^{s} \otimes \Lambda^{a p^{j}-s}, S^{a(j)}\right) \Longrightarrow 0 .
$$

Proceeding by induction, we see that Proposition 5.2 implies that only 3 columns are possibly non-zero: $s=0,(a-1) \cdot p^{j}, a p^{j}$. To verify $\operatorname{Ext}_{\mathcal{P}}^{*}\left(\Lambda^{a p^{j}}, S^{a(j)}\right)=0$, it suffices to verify that the differential

$$
d_{p^{j}}: \operatorname{Ext}^{t}\left(\Gamma^{(a-1) \cdot p^{j}} \otimes \Lambda^{p^{j}}, S^{a(j)}\right) \rightarrow E x t^{t-\left(p^{j}-1\right)}\left(\Gamma^{a p^{j}}, S^{a(j)}\right)
$$


is an isomorphism. To analyze $d_{p^{j}}$, we observe that multiplication in the symmetric algebra defines a canonical homomorphism of complexes $K o_{p^{j}}^{\bullet} \otimes S^{(a-1) \cdot p^{j}} \rightarrow K o_{a \cdot p^{j}}^{\bullet}$ which has the following form

$$
\begin{aligned}
& 0 \quad \rightarrow \quad \Lambda^{p^{j}} \otimes S^{(a-1) \cdot p^{j}} \rightarrow \cdots \quad \rightarrow \quad S^{p^{j}} \otimes S^{(a-1) \cdot p^{j}} \rightarrow 0
\end{aligned}
$$

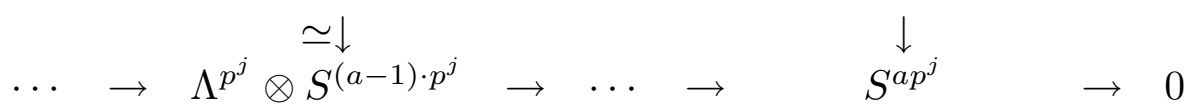

and whose dual has the form

$$
\begin{aligned}
& 0 \rightarrow \quad \Gamma^{a \cdot p^{j}} \quad \rightarrow \quad \cdots \quad \rightarrow \quad \Gamma^{(a-1) \cdot p^{j}} \otimes \Lambda^{p^{j}} \quad \rightarrow \quad \cdots
\end{aligned}
$$

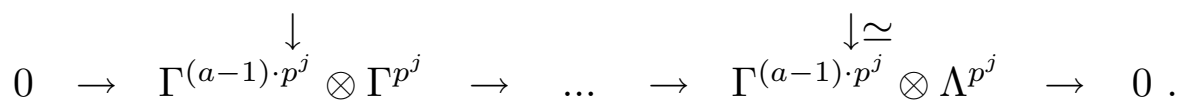

Applying Proposition 5.2, we see that the homomorphism of hypercohomology spectral sequences induced by (5.4.3) gives the following commutative diagram:

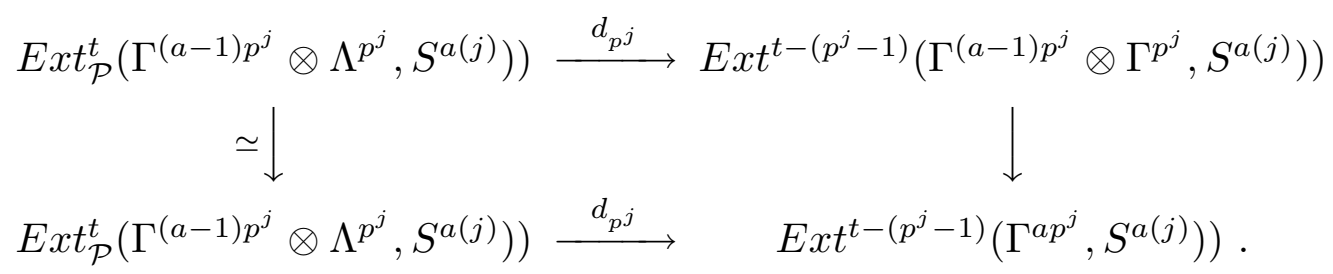

The upper $d_{p^{j}}$ is an isomorphism since it arises from the spectral sequence of the complex $\Gamma^{(a-1) \cdot p^{j}} \otimes K o_{p^{j}}^{\#}$, which converges to zero and has only two non zero columns. Moreover the right vertical arrow is also an isomorphism: if $t \neq p^{j}-1$ then both Ext-groups are zero, whereas the map on the Hom-groups coincides (in view of Corollary 2.12) with the homomorphism

$$
S^{a}\left(\left(k^{2}\right)^{(j)}\right)^{\left((a-1) p^{j}, p^{j}\right)}=S^{a-1}\left(k^{(j)}\right) \otimes k^{(j)} \stackrel{\text { mult }}{\longrightarrow} S^{a}\left(k^{(j)}\right)
$$

which is clearly an isomorphism. This implies that the bottom differential $d_{p^{j}}$ is also an isomorphism.

We now verify the asserted computation for $\operatorname{Ext}_{\mathcal{P}}^{n}\left(S^{a p^{j}}, S^{a(j)}\right)$. Again, we proceed by induction on $a$, the case $a=0$ being trivial. Consider now the first hypercohomology spectral sequence corresponding to the complex $K o_{a p^{j}}^{\bullet}$

$$
E_{1}^{s, t}=\operatorname{Ext} t_{\mathcal{P}}^{t}\left(S^{a p^{j}-s} \otimes \Lambda^{s}, S^{a(j)}\right) \Longrightarrow 0 .
$$

Proposition 5.2 and the computation of $\operatorname{Ext}_{\mathcal{P}}^{*}\left(\Lambda^{a p^{j}}, S^{a(j)}\right)$ show that the only possibly non-zero columns are $s=0, p^{j}$. Thus the differential

$$
\begin{gathered}
\left.\left.d_{p^{j}}: \operatorname{Ext}_{\mathcal{P}}^{t}\left(S^{a p^{j}}, S^{a(j)}\right)\right) \rightarrow \operatorname{Ext}_{\mathcal{P}}^{t-\left(p^{j}-1\right)}\left(S^{(a-1) \cdot p^{j}} \otimes \Lambda^{p^{j}}, S^{a(j)}\right)\right)= \\
=\operatorname{Ext}_{\mathcal{P}}^{t-2\left(p^{j}-1\right)}\left(S^{(a-1) \cdot p^{j}}, S^{a-1(j)}\right)
\end{gathered}
$$

is an isomorphism.

The following proposition (for $j=1$ ) together with (5.2) implies that the upper left map of Diagram 5.1 is an isomorphism . 
Proposition 5.5. Multiplication $S^{(a-1) \cdot p^{j}} \otimes S^{p^{j}} \rightarrow S^{a p^{j}}$ induces an isomorphism of Ext-groups

$$
\operatorname{Ext}_{\mathcal{P}}^{*}\left(S^{a p^{j}}, S^{a(j)}\right) \stackrel{\simeq}{\longrightarrow} \operatorname{Ext}_{\mathcal{P}}^{*}\left(S^{(a-1) \cdot p^{j}} \otimes S^{p^{j}}, S^{a(j)}\right) .
$$

Proof. The homomorphism of complexes $K o_{p^{j}}^{\bullet} \otimes S^{(a-1) \cdot p^{j}} \rightarrow K o_{a p^{j}}^{\bullet}$ made explicit in (5.4.2) provides the following analogue of (5.4.4)

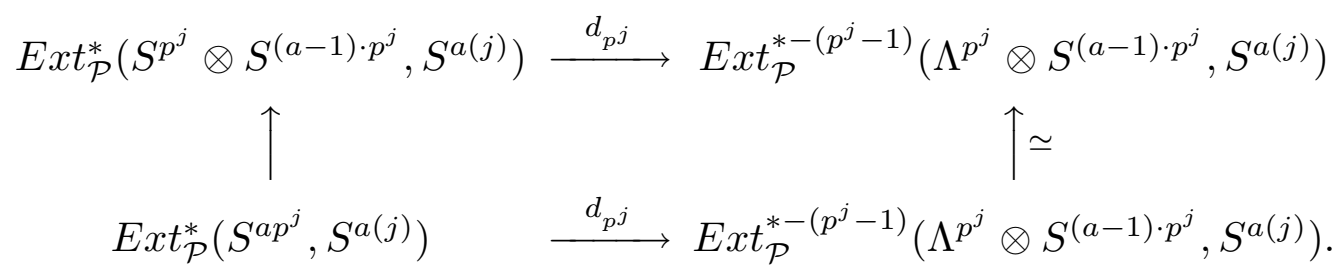

Both differentials $d_{p^{r}}$ are isomorphisms since both spectral sequences have only two non zero columns (as one sees from (5.2) and (5.4)). Thus the left vertical arrow is also an isomorphism.

We conclude our discussion of Diagram 5.1 with the following verification that the bottom horizontal map is an isomorphism.

Proposition 5.6. The canonical map $I^{(1)} \rightarrow S^{p}$ induces an isomorphism

$$
\operatorname{Ext}_{\mathcal{P}}^{2(p-1)}\left(S^{p}, I^{(1)}\right) \stackrel{\simeq}{\longrightarrow} \operatorname{Ext}_{\mathcal{P}}^{2(p-1)}\left(I^{(1)}, I^{(1)}\right) .
$$

Proof. Consider the hypercohomology spectral sequence obtained by applying the functor $\mathrm{RHom}_{\mathcal{P}}\left(-, I^{(1)}\right)$ to the exact sequence

$$
0 \rightarrow I^{(1)} \rightarrow S^{p} \rightarrow \Gamma^{p} \rightarrow I^{(1)} \rightarrow 0
$$

Theorem 4.5 provides us with a computation of $\operatorname{Ext}_{\mathcal{P}}^{*}\left(I^{(1)}, I^{(1)}\right)$, the projectivity of $\Gamma^{p}$ determines $\operatorname{Ext}_{\mathcal{P}}^{*}\left(\Gamma^{p}, I^{(1)}\right)$, and Proposition 5.4 determines $\operatorname{Ext}_{\mathcal{P}}^{*}\left(S^{p}, I^{(1)}\right)$. Thus, the $E_{1}$-term of this hypercohomology spectral sequence has the following form:

$\begin{array}{cccccc}k & & 0 & k & \rightarrow & k \\ 0 & & 0 & 0 & & 0 \\ k & & 0 & 0 & & k \\ \vdots & & \vdots & \vdots & & \vdots \\ 0 & & 0 & 0 & & 0 \\ k & & 0 & 0 & & k \\ 0 & & 0 & 0 & & 0 \\ k \stackrel{\sim}{\longrightarrow} & k & 0 & & k\end{array}$

We conclude immediately that $\operatorname{Ext}_{\mathcal{P}}^{2(p-1)}\left(S^{p}, I^{(1)}\right) \rightarrow \operatorname{Ext}_{\mathcal{P}}^{2(p-1)}\left(I^{(1)}, I^{(1)}\right)$ (the upper right map of the above diagram) is an isomorphism.

By Corollary 4.8, $e_{r}^{p-1}$ spans $\operatorname{Ext}_{\mathcal{P}}^{2(p-1) p^{r-1}}\left(I^{(r)}, I^{(r)}\right)$. Thus, the commutativity of Diagram 5.1 together with the isomorphisms proved above implies the following corollary. 
Corollary 5.7. The image of $\left(e_{1}^{p-1}\right)^{\otimes p^{r-1}} \in\left(\operatorname{Ext}_{\mathcal{P}}^{2(p-1)}\left(I^{(1)}, I^{(1)}\right)\right)^{\otimes p^{r-1}}$ in $\operatorname{Ext}_{\mathcal{P}}^{2(p-1) p^{r-1}}\left(\Gamma^{p^{r-1}(1)}, S^{p^{r-1}(1)}\right) \quad$ is a scalar multiple of the image of $e_{r}^{p-1} \in \operatorname{Ext}_{\mathcal{P}}^{2(p-1) p^{r-1}}\left(I^{(r)}, I^{(r)}\right)$ in $\operatorname{Ext}_{\mathcal{P}}^{2(p-1) p^{r-1}}\left(\Gamma^{p^{r-1}(1)}, S^{p^{r-1}(1)}\right)$.

Remark 5.8 We did not compute the coefficient which relates the images of $\left(e_{1}^{p-1}\right)^{\otimes p^{r-1}}$ and $e_{r}^{p-1}$ in $\operatorname{Ext}_{\mathcal{P}}^{2(p-1) p^{r-1}}\left(\Gamma^{p^{r-1}(1)}, S^{p^{r-1}(1)}\right)$. Most probably it is equal to 1 . One thing that is evident is that this coefficient belongs to $\mathbb{F}_{p}$, moreover it will be clear from the results in $\S 6$ that this coefficient is at least non-zero. Our original expectation was that images of $e_{1}^{\otimes p^{r-1}}$ and $e_{r}$ in $\operatorname{Ext}_{\mathcal{P}}^{2 p^{r-1}}\left(\Gamma^{p^{r-1}(1)}, S^{p^{r-1}(1)}\right)$ coincide. The above remarks show that this is true for $p=2$, however we don't know if this result holds in general. To facilitate this kind of comparison it would have been extremely useful to be able to compute the $\operatorname{Ext}$-groups $\operatorname{Ext}_{\mathcal{P}}^{*}\left(\Gamma^{n(1)}, S^{n(1)}\right)$.

\section{$\S 6$. NON VANishing of $\left.e_{r}\right|_{\left(G L_{n}\right)_{(1)}}$}

The purpose of this section is to complete the proof of Theorem 1.2 (and thus of Theorem 1.1) by verifying non vanishing of the restriction of $e_{r}$ to $\left(G L_{n}\right)_{(1)}$. We first establish this non vanishing for $n \geq p$ and then extend this result to all $n \geq 2$ using the following compatibility property mentioned above in Remark 1.2.1.

Lemma 6.1. Assume that $n \leq m$. Denote by $i$ the standard embeding $G L_{n} \hookrightarrow$ $G L_{m}$ and by $p: g l_{m} \rightarrow g l_{n}$ denote the $G L_{n}$-equivariant projection sending the matrix $\alpha=\left(a_{i j}\right)_{i, j=1}^{m}$ to its upper left corner $p(\alpha)=\left(a_{i j}\right)_{i, j=1}^{n}$. Consider the induced map on rational cohomology groups

$$
H^{*}\left(G L_{m}, g l_{m}^{(r)}\right) \stackrel{i^{*}}{\longrightarrow} H^{*}\left(G L_{n}, g l_{m}^{(r)}\right) \stackrel{\left(p^{(r)}\right)_{*}}{\longrightarrow} H^{*}\left(G L_{n}, g l_{n}^{(r)}\right)
$$

The image under this homomorphism of $e_{r} \in H^{2 p^{r-1}}\left(G L_{m}, g l_{m}^{(r)}\right)$ equals $e_{r} \in$ $H^{2 p^{r-1}}\left(G L_{n}, g l_{n}^{(r)}\right)$.

Proof. This follows immediately from Lemma 3.14.

For $n \geq p$ we will show non vanishing of the restriction of $e_{r}$ to $\left(G L_{n}\right)_{(1)}$ by restricting it further to $\left(\mathbf{G}_{\mathbf{a}}\right)_{(1)}$ for an appropriately chosen subgroup $\mathbf{G}_{\mathbf{a}} \subset G L_{n}$.

The cohomology ring of the infinitesimal group scheme $\left(\mathbf{G}_{\mathbf{a}}\right)_{(1)}$ is easy to compute. The coordinate Hopf algebra $k\left[\left(\mathbf{G}_{\mathbf{a}}\right)_{(1)}\right]$ coincides with $k[T] / T^{p}$ and the comultiplcation takes $T$ to $T \otimes 1+1 \otimes T$. Let $B=k\left[\left(\mathbf{G}_{\mathbf{a}}\right)_{(1)}\right]^{\#}$ be the dual Hopf algebra and let $y \in B$ be defined by $y\left(T^{i}\right)=\delta_{1, i}$. One checks immediately that $B=k[y]=k[Y] / Y^{p}$. The argument used in the proof of Lemma 3.4 shows that the category of rational $\left(\mathbf{G}_{\mathbf{a}}\right)_{(1)}$-modules coincides with the category of $B$-modules and hence for any rational $\left(\mathbf{G}_{\mathbf{a}}\right)_{(1)}$-module $M$ we have:

$$
H^{*}\left(\left(\mathbf{G}_{\mathbf{a}}\right)_{(1)}, M\right)=\operatorname{Ext}_{B}^{*}(k, M)=H^{*}(B, M) .
$$

Furthermore the algebra $B$ may be identified with the group algebra of a cyclic group of order $p$ generated by $1+y \in B^{*}$. Thus $H^{*}(B, M)=H^{*}(\mathbb{Z} / p, M)$, and we may apply the well-known results concerning cohomology of a cyclic group. We recall briefly the main facts in terms convenient for our purposes. 
First of all, $k$ has a canonical 2-periodic projective $B$-resolution

$$
0 \leftarrow k \leftarrow B \stackrel{y}{\longleftarrow} B \stackrel{y^{p-1}}{\longleftarrow} B \leftarrow \ldots
$$

Using this resolution we get the standard computation of cohomology

$$
H^{i}(B, M)=\left\{\begin{aligned}
y^{M} & \text { if } i=0 \\
{ }_{y} M / y^{p-1} M & \text { if } i=2 l>0 \\
y^{p-1} M / y M & \text { if } i=2 l+1
\end{aligned}\right.
$$

The periodicity isomorphism $H^{i}(B, M) \stackrel{\sim}{\longrightarrow} H^{i+2}(B, M)$ coincides with multiplication by the generator $\beta \in H^{2}(B, k)$ represented by the extension

$$
0 \leftarrow k \leftarrow B \stackrel{y}{\longleftarrow} B \stackrel{y^{p-1}}{\longleftarrow} k \leftarrow 0 .
$$

In particular the cohomology ring $H^{\mathrm{ev}}(B, k)$ coincides with the polynomial algebra $k[\beta]$.

Consider $k^{n}$ as a trivial $B$-module and denote by $\epsilon_{i} \quad(1 \leq i \leq n)$ the standard basis of $k^{n}$. The vector space $\operatorname{Ext}_{B}^{*}\left(k^{n}, k^{m}\right)$ may be identified with the set of matrices $M_{m, n}\left(H^{*}(B, k)\right)$. After this identification the Yoneda pairing

$$
\operatorname{Ext}_{B}^{*}\left(k^{n}, k^{m}\right) \times \operatorname{Ext}_{B}^{*}\left(k^{l}, k^{n}\right) \rightarrow \operatorname{Ext}_{B}^{*}\left(k^{l}, k^{m}\right)
$$

becomes the usual multiplication of matrices.

Let $0 \rightarrow k^{m} \stackrel{h}{\rightarrow} W \stackrel{g}{\rightarrow} V \stackrel{f}{\rightarrow} k^{n} \rightarrow 0$ be an extension of $B$-modules. The corresponding element from $\operatorname{Ext}_{B}^{2}\left(k^{n}, k^{m}\right)=M_{m, n}\left(H^{2}(B, k)\right)$ may be written in the form $\gamma \cdot \beta$ for a unique matrix $\gamma \in M_{m, n}(k)$. The components of $\gamma$ may be computed in the following way: choose $v_{j} \in V$ such that $f\left(v_{j}\right)=\epsilon_{j}$, next choose $w_{j} \in W$ such that $g\left(w_{j}\right)=y \cdot v_{j}$. The element $y^{p-1} \cdot w_{j}$ may be written in the form $h\left(s_{j}\right)$ for a unique column $s_{j} \in k^{m}$ and $s_{j}$ coincides with the $j$-th column of $\gamma$.

Theorem 6.2. For any $n \geq 2, r \geq 1$ the restriction of $e_{r} \in H^{2 p^{r-1}}\left(G L_{n}, g l_{n}^{(r)}\right)$ to the infinitesimal subgroup $\left(G L_{n}\right)_{(1)}$ is nontrivial.

Proof. Assume first that $n \geq p$. Set $\alpha=e_{1,2}+\ldots+e_{p-1, p}$, where $e_{i, j} \in M_{n}(k)$ has 1 in the $(i, j)$ position and 0's elsewhere. Note that $\alpha^{p-1}=e_{1, p}, \alpha^{p}=0$. Thus for any $t \in k$ the exponent $\exp (t \alpha)=\sum_{i=0}^{\infty} \frac{(t \alpha)^{i}}{i !}$ is well-defined and

$$
\exp _{\alpha}: t \mapsto \exp (t \alpha)
$$

defines group scheme homomorphisms (actually closed embedings)

$$
\exp _{\alpha}: \mathbf{G}_{\mathbf{a}} \rightarrow G L_{n}, \quad\left(\exp _{\alpha}\right)_{(1)}:\left(\mathbf{G}_{\mathbf{a}}\right)_{(1)} \rightarrow\left(G L_{n}\right)_{(1)} .
$$

The homomorphism $\left(\exp _{\alpha}\right)_{(1)}$ makes the standard $G L_{n}$-module $k^{n}$ into a rational $\left(\mathbf{G}_{\mathbf{a}}\right)_{(1)}$-module and hence into a $B$-module. One verifies readily that the action of $y$ on $k^{n}$ looks as follows:

$$
y \cdot \epsilon_{1}=0, y \cdot \epsilon_{i}=\epsilon_{i-1}(2 \leq i \leq p), y \cdot \epsilon_{i}=0(p<i \leq n) .
$$


Restricting $\left.e_{1}\right|_{\left(G L_{n}\right)_{(1)}}$ to $\left(\mathbf{G}_{\mathbf{a}}\right)_{(1)}$ via the homomorphism $\left(\exp _{\alpha}\right)_{(1)}$ we get an element in $\operatorname{Ext}_{B}^{2}\left(\left(k^{n}\right)^{(1)},\left(k^{n}\right)^{(1)}\right)$ represented by the extension

$$
0 \rightarrow\left(k^{n}\right)^{(1)} \rightarrow S^{p}\left(k^{n}\right) \rightarrow \Gamma^{p}\left(k^{n}\right) \rightarrow\left(k^{n}\right)^{(1)} \rightarrow 0 .
$$

Note further that $\left(k^{n}\right)^{(1)}$ is a trivial $B$-module with basis $\left\{\epsilon_{i}^{(1)}\right\}_{i=1}^{n}$, so that $\operatorname{Ext}_{B}^{2}\left(\left(k^{n}\right)^{(1)},\left(k^{n}\right)^{(1)}\right)$ may be identified with $M_{n}\left(H^{2}(B, k)\right)$. A straightforward computation using the procedure described above proves the following fact

(6.2.1). $\left(\left(\exp _{\alpha}\right)_{(1)}\right)^{*}\left(\left.e_{1}\right|_{\left(G L_{n}\right)_{(1)}}\right)=\alpha \cdot \beta \in M_{n}\left(H^{2}(B, k)\right)$.

As an immediate corollary of (6.2.1) we get that $\left(\left(\exp _{\alpha}\right)_{(1)}\right)^{*}\left(\left.e_{1}^{p-1}\right|_{\left(G L_{n}\right)_{(1)}}\right)=$ $e_{1, p} \cdot \beta^{p-1}$ and hence the image of $\left(\left.e_{1}^{p-1}\right|_{\left(\mathbf{G}_{\mathbf{a}}\right)_{(1)}}\right)^{\otimes p}$ in

$$
\begin{aligned}
\operatorname{Ext}_{B}^{2(p-1) p^{r-1}}\left(\Gamma^{p^{r-1}}\left(\left(k^{n}\right)^{(1)}\right), S^{p^{r-1}}\left(\left(k^{n}\right)^{(1)}\right)\right)= \\
=\operatorname{Hom}_{k}\left(\Gamma^{p^{r-1}}\left(\left(k^{n}\right)^{(1)}\right), S^{p^{r-1}}\left(\left(k^{n}\right)^{(1)}\right)\right) \otimes H^{2(p-1) p^{r-1}}(B, k)
\end{aligned}
$$

equals $\gamma \cdot \beta^{(p-1) p^{r-1}}$, where $\gamma$ is the following $k$-linear map

$$
\Gamma^{p^{r-1}}\left(\left(k^{n}\right)^{(1)}\right) \hookrightarrow\left(\left(k^{n}\right)^{(1)}\right)^{\otimes p^{r-1}} \stackrel{e_{1, p}^{\otimes p^{r-1}}}{\longrightarrow}\left(\left(k^{n}\right)^{(1)}\right)^{\otimes p^{r-1}} \rightarrow S^{p^{r-1}}\left(\left(k^{n}\right)^{(1)}\right) .
$$

A trivial verification shows that $\gamma \neq 0$ and hence $\gamma \cdot \beta^{(p-1) p^{r-1}} \neq 0$. On the other hand Corollary 5.7 shows that $\gamma \cdot \beta^{(p-1) p^{r-1}}$ is a scalar factor of the image of $\left.e_{r}^{p-1}\right|_{\left(G L_{n}\right)_{(1)}}$ in the corresponding group. Thus $\left.e_{r}^{p-1}\right|_{\left(G L_{n}\right)_{(1)}} \neq 0$ and hence $\left.e_{r}\right|_{\left(G L_{n}\right)_{(1)}} \neq 0$.

Assume now that $2 \leq n<p$. Denote by $i: G L_{n} \hookrightarrow G L_{p+1}$ the standard embeding and by $q: g l_{p+1} \rightarrow g l_{n}$ the standard $G L_{n}$-equivariant projection. Lemma 6.1 gives us a commutative diagram

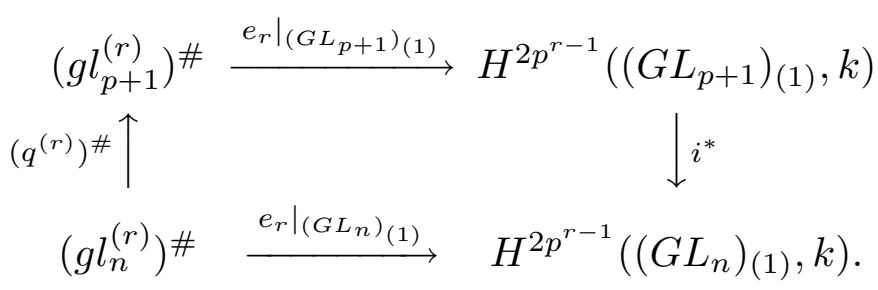

According to Lemmas 1.4 and 1.7, we conclude that the $k$-linear map $\left.e_{r}\right|_{\left(G L_{p+1}\right)_{(1)}}$ coincides (up to a non-zero scalar factor) with the composition

$$
\left(g l_{p+1}^{(r)}\right)^{\#} \rightarrow S^{p^{r-1}}\left(\left(g l_{p+1}^{(1)}\right)^{\#}\right) \rightarrow H^{2 p^{r-1}}\left(\left(G L_{p+1}\right)_{(1)}, k\right)
$$

where the first arrow coincides with raising to the power $p^{r-1}$ and the second one is the edge homomorphism in the May spectral sequence. Since both these arrows are clearly functorial on the category of infinitesimal groups of height one we get 
(using the same notation $i$ for the induced map of the Lie algebras $g l_{n} \hookrightarrow g l_{p+1}$ ) one more commutative diagram

$$
\begin{aligned}
& \left(g l_{p+1}^{(r)}\right)^{\#} \longrightarrow S^{p^{r-1}}\left(\left(g l_{p+1}^{(1)}\right)^{\#}\right) \longrightarrow H^{2 p^{r-1}}\left(\left(G L_{p+1}\right)_{(1)}, k\right) \\
& \downarrow\left(i^{(r)}\right)^{\#} \quad \downarrow S^{p^{r-1}}\left(\left(i^{(1)}\right)^{\#}\right) \downarrow i^{*} \\
& \left(g l_{n}^{(r)}\right)^{\#} \longrightarrow S^{p^{r-1}}\left(\left(g l_{n}^{(1)}\right)^{\#}\right) \longrightarrow H^{2 p^{r-1}}\left(\left(G L_{n}\right)_{(1)}, k\right) \text {. }
\end{aligned}
$$

Since the composition $\left(i^{(r)}\right)^{\#} \cdot\left(q^{(r)}\right)^{\#}=\left((q i)^{(r)}\right)^{\#}$ is the identity map we conclude from the above diagrams that $\left.e_{r}\right|_{\left(G L_{n}\right)_{(1)}}$ coincides up to a non-zero scalar factor with the composition

$$
\left(g l_{n}^{(r)}\right)^{\#} \rightarrow S^{p^{r-1}}\left(\left(g l_{n}^{(1)}\right)^{\#}\right) \rightarrow H^{2 p^{r-1}}\left(\left(G L_{n}\right)_{(1)}, k\right) .
$$

To end the proof it suffices now to establish the following Lemma

Lemma 6.3. For any $n \geq 2, r \geq 1$, the composition

$$
\left(g l_{n}^{(r)}\right)^{\#} \rightarrow S^{p^{r-1}}\left(\left(g l_{n}^{(1)}\right)^{\#}\right) \rightarrow H^{2 p^{r-1}}\left(\left(G L_{n}\right)_{(1)}, k\right)
$$

is non-zero.

Proof. Consider the standard embeding $\mathbf{G}_{\mathbf{a}} \hookrightarrow G L_{2} \hookrightarrow G L_{n}: t \mapsto 1_{n}+t e_{1,2}$. Using once again the naturality of the involved homomorphisms we get a commutative diagram

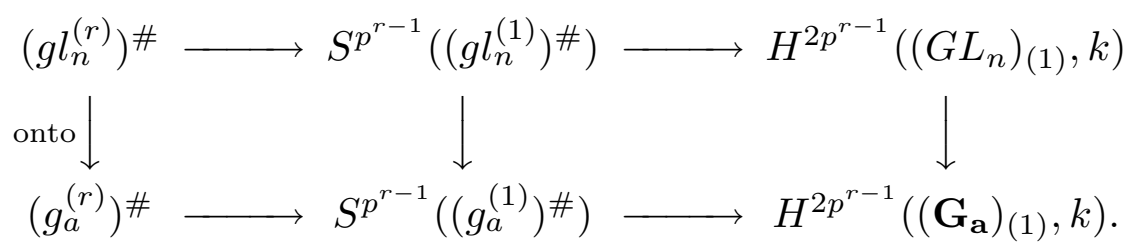

Thus it suffices to check non-triviality of the composition of arrows in the bottom row. The one-dimensional vector space $g_{a}^{\#}$ may be canonically identified with $\mathfrak{M} / \mathfrak{M}^{2}$, where $\mathfrak{M} \subset k\left[\left(\mathbf{G}_{\mathbf{a}}\right)\right]$ is the augmentation ideal. Thus $g_{a}^{\#}=k \cdot t$ (where $t=T \bmod \mathfrak{M}^{2}$ ) and hence $\left(g_{a}^{(r)}\right)^{\#}=\left(g_{a}^{\#}\right)^{(r)}=k \cdot t^{(r)}$. The image of $t^{(r)}$ in $S^{p^{r-1}}\left(\left(g_{a}^{\#}\right)^{(1)}\right)$ equals $\left(t^{(1)}\right)^{p^{r-1}}$. Further the May spectral sequence for the infinitesimal group scheme of height one $\left(\mathbf{G}_{\mathbf{a}}\right)_{(1)}$ gives us an exact sequence $\left(g_{a}^{(1)}\right)^{\#} \rightarrow H^{2}\left(\left(\mathbf{G}_{\mathbf{a}}\right)_{(1)}, k\right) \rightarrow H^{2}\left(g_{a}, k\right)=0$. Thus the image of $t^{(1)}$ in $H^{2}\left(\left(\mathbf{G}_{\mathbf{a}}\right)_{(1)}, k\right)$ is a non zero scalar multiple of $\beta$ (actually $\beta$ itself) and hence the image of $\left(t^{(1)}\right)^{p^{r-1}}$ in $H^{2 p^{r-1}}\left(\left(\mathbf{G}_{\mathbf{a}}\right)_{(1)}, k\right)$ is a non zero scalar multiple of $\beta^{p^{r-1}}$.

$$
\S 7 \text { Computation of } H_{*}\left(G L\left(\mathbf{F}_{q}\right), M\left(\mathbf{F}_{q}\right)\right) .
$$

One pleasing consequence of the computation of rational cohomology of $G L_{n}$ with coefficients in the twisted adjoint representation achieved in the previous sections is a purely algebraic computation of the stable homology of the general linear 
group over a finite field with coefficients in the adjoint representation. This computation, originally made by M. Bökstedt[B], has previously utilized methods arising from geometric topology.

For a field $k$ we denote by $G L_{n}(k)$ the discrete group of invertible $n \times n$ matrices over $k$ and by $M_{n}(k)$ we denote the adjoint representation of $G L_{n}(k)$ (so that $G L_{n}(k)$ acts on $M_{n}(k)$ via conjugation of matrices). We embed $G L_{n}(k)$ into $G L_{n+1}(k)$ (and $M_{n}(k)$ into $M_{n+1}(k)$ ) in a standard way and we set $G L(k)=$ $\lim G L_{n}(k), \quad M(k)=\lim M_{n}(k)$. The main result of this section (Theorem 7.6 below) computes the homology groups $H_{*}(G L(k), M(k))$ in case $k$ is a finite field.

We start with a few preliminary remarks. First of all since group homology commutes with direct limits we conclude that $H_{i}(G L(k), M(k))=$ $=\lim _{\rightarrow} H_{i}\left(G L_{n}(k), M_{n}(k)\right)$. Furthermore a stability theorem of W. Dwyer[Dw] shows that there exists an increasing integer function $m \mapsto N(m)$ with the following property: for any associative ring with unit $R$ and any $n \geq N(m)$ the natural homomorphisms

$$
\begin{aligned}
H_{i}\left(G L_{n}(R), M_{n}(R)\right) & \rightarrow H_{i}\left(G L_{n+1}(R), M_{n+1}(R)\right) \\
H_{i}\left(G L_{n}(R), \mathbb{Z}\right) & \rightarrow H_{i}\left(G L_{n+1}(R), \mathbb{Z}\right)
\end{aligned}
$$

are isomorphisms for all $i \leq m$. Thus $H_{i}(G L(k), M(k))=H_{i}\left(G L_{n}(k), M_{n}(k)\right)$ for all $n \geq N(i)$ (and all fields $k$ ). Moreover for $i>0$ and $n \geq N(i)$ we have $H_{i}\left(G L_{n}\left(\mathbb{F}_{q}\right), \mathbb{F}_{q}\right)=H_{i}\left(G L\left(\mathbb{F}_{q}\right), \mathbb{F}_{q}\right)=0$. Here we consider $\mathbb{F}_{q}$ as a trivial $G L\left(\mathbb{F}_{q}\right)$ module and use a theorem of Quillen $[\mathrm{Q}]$, giving the vanishing of $\tilde{H}_{*}\left(G L\left(\mathbb{F}_{q}\right), \mathbb{F}_{q}\right)$.

The homology groups under consideration fit into the following more general picture. Let $A$ be an associative ring with unit. The group of units $A^{*}$ acts on the additive group of $A$ by conjugation, so that we may consider the homology groups $H_{*}\left(A^{*}, A\right)$. Let $\phi: A \rightarrow B$ be a ring homomorphism (not necessarily taking $1_{A}$ to $\left.1_{B}\right)$. It defines a homomorphism on the group of units $A^{*} \rightarrow B^{*}$ : $u \mapsto 1_{B}+\phi\left(u-1_{A}\right)$, which is compatible with actions of $A^{*}$ and $B^{*}$ on $A$ and $B$ respectively and hence we get natural homomorphisms in group homology

$$
\phi_{*}: H_{*}\left(A^{*}, A\right) \rightarrow H_{*}\left(B^{*}, B\right) .
$$

Note that $\phi_{*}=\phi_{*}^{\prime}$ provided that the ring homomorphisms $\phi, \phi^{\prime}: A \rightarrow B$ are conjugate (by an element of $B^{*}$ ).

Let $k / k_{0}$ be a finite extension of fields of degree $d=\left[k: k_{0}\right]$. Choosing a basis for $k$ over $k_{0}$ we get an embeding $k \hookrightarrow M_{d}\left(k_{0}\right)$, defined uniquely up to a conjugation (by an element of $\left.G L_{d}\left(k_{0}\right)=\left(M_{d}\left(k_{0}\right)\right)^{*}\right)$. Further for all $n>0$ we get induced embedings $M_{n}(k) \hookrightarrow M_{n}\left(M_{d}\left(k_{0}\right)\right)=M_{n d}\left(k_{0}\right)$ also well defined up to conjugation and hence canonical homomorphisms in group homology

$$
H_{*}\left(G L_{n}(k), M_{n}(k)\right) \rightarrow H_{*}\left(G L_{n d}\left(k_{0}\right), M_{n d}\left(k_{0}\right)\right) .
$$

One checks easily that the following diagram commutes

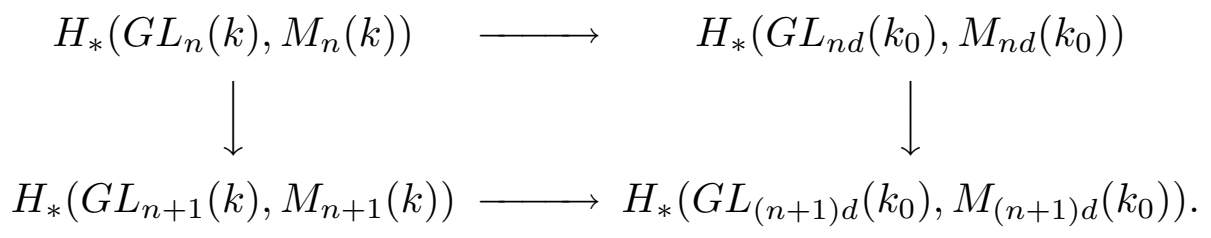


Taking now $n \geq N(i)$, we get a homomorphism

$$
\begin{aligned}
\operatorname{Tr}_{k / k_{0}}: H_{i}(G L(k), M(k))=H_{i}\left(G L_{n}(k), M_{n}(k)\right) & \rightarrow H_{i}\left(G L_{n d}\left(k_{0}\right), M_{n d}\left(k_{0}\right)\right)= \\
& =H_{i}\left(G L\left(k_{0}\right), M\left(k_{0}\right)\right)
\end{aligned}
$$

independent of the choice of $n(\geq N(i))$.

Note also that whenever $k / k_{0}$ is a Galois extension we have an evident action of the Galois group $\operatorname{Gal}\left(k / k_{0}\right)$ on $H_{*}\left(G L_{n}(k), M_{n}(k)\right)$ and the image of $H_{*}\left(G L_{n}\left(k_{0}\right), M_{n}\left(k_{0}\right)\right)$ in $H_{*}\left(G L_{n}(k), M_{n}(k)\right)$ is contained in the subgroup of $\operatorname{Gal}\left(k / k_{0}\right)$-invariants.

Proposition 7.1. Let $k / k_{0}$ be an extension of finite fields. Then the composition

$$
H_{m}\left(G L\left(k_{0}\right), M\left(k_{0}\right)\right) \stackrel{\mathrm{can}}{\longrightarrow} H_{m}(G L(k), M(k)) \stackrel{T r_{k / k_{0}}}{\longrightarrow} H_{m}\left(G L\left(k_{0}\right), M\left(k_{0}\right)\right)
$$

coincides with multiplication by $d=\left[k: k_{0}\right]$, whereas the composition

$$
H_{m}(G L(k), M(k)) \stackrel{T r_{k / k_{0}}}{\longrightarrow} H_{m}\left(G L\left(k_{0}\right), M\left(k_{0}\right)\right) \stackrel{\text { can }}{\longrightarrow} H_{m}(G L(k), M(k))
$$

coincides with $\sum_{\sigma \in \operatorname{Gal}\left(k / k_{0}\right)} \sigma_{*}$.

Proof. Choose any $n \geq N(m)$. The composition

$$
H_{m}(G L(k), M(k)) \stackrel{\text { can } \cdot \operatorname{Tr}_{k / k_{0}}}{\longrightarrow} H_{m}(G L(k), M(k))
$$

is induced by a ring homomorphism $M_{n}(k) \rightarrow M_{n d}(k)$, which is the matrix extension of the embeding $k \hookrightarrow M_{d}(k)=\operatorname{End}_{k}\left(k \otimes_{k_{0}} k\right)$. Here we consider $k \otimes_{k_{0}} k$ as a $d$-dimensional vector space over $k$ via the left action of $k$ on $k \otimes_{k_{0}} k$ and we embed $k$ into $\operatorname{End}_{k}\left(k \otimes_{k_{0}} k\right)$ using the right action of $k$ on $k \otimes_{k_{0}} k$. It's well-known that the ring $k \otimes_{k_{0}} k$ is canonically isomorphic to $\prod_{\sigma \in \operatorname{Gal}\left(k / k_{0}\right)} k$. For any $x \in k$ this isomorphism takes $x \otimes 1$ to $\prod_{\sigma} x$ and $1 \otimes x$ to $\prod_{\sigma} x^{\sigma}$. This shows that the embeding $k \hookrightarrow M_{d}(k)$ is conjugate to the embeding which takes $x \in k$ to $\operatorname{diag}\left(x^{\sigma}\right)_{\sigma \in \operatorname{Gal}\left(k / k_{0}\right)}$. Taking matrix extensions we conclude that the embeding $M_{n}(k) \hookrightarrow M_{n d}(k)$ is conjugate to the embeding of the form $\alpha \mapsto \operatorname{diag}\left(\alpha^{\sigma}\right)_{\sigma \in \operatorname{Gal}\left(k / k_{0}\right)}$.

The same reasoning shows that the composition

$$
H_{m}\left(G L\left(k_{0}\right), M\left(k_{0}\right)\right) \stackrel{T r_{k / k_{0}} \cdot \operatorname{can}}{\longrightarrow} H_{m}\left(G L\left(k_{0}\right), M\left(k_{0}\right)\right)
$$

is induced by a ring embeding which is conjugate to the embeding of the form $\alpha \mapsto \operatorname{diag}(\alpha)_{\sigma \in \operatorname{Gal}\left(k / k_{0}\right)}$. Now it suffices to use the following Lemma

Lemma 7.1.1. Let $k$ be a finite field. Assume that we are given two ring homomorphisms $\phi: M_{n}(k) \rightarrow M_{n_{1}}(k), \psi: M_{n}(k) \rightarrow M_{n_{2}}(k)$, where $n, n_{1}, n_{2} \geq$ $N(m)$. Then the homomorphism $H_{m}(G L(k), M(k)) \rightarrow H_{m}(G L(k), M(k))$ induced 
by $\operatorname{diag}(\phi, \psi): M_{n}(k) \rightarrow M_{n_{1}+n_{2}}(k)$ coincides with the sum of homomorphisms induced by $\phi$ and $\psi$ respectively.

Proof. The ring homomorpism $\operatorname{diag}(\phi, \psi)$ factors via a subring $A=M_{n_{1}}(k) \times$ $M_{n_{2}}(k) \subset M_{n_{1}+n_{2}}(k)$. Furthermore using the Kunneth formula we get:

$$
\begin{aligned}
& H_{m}\left(A^{*}, A\right)=H_{m}\left(G L_{n_{1}}(k) \times G L_{n_{2}}(k), M_{n_{1}}(k) \oplus M_{n_{2}}(k)\right)= \\
& =H_{m}\left(G L_{n_{1}}(k) \times G L_{n_{2}}(k), M_{n_{1}}(k)\right) \oplus H_{m}\left(G l_{n_{1}}(k) \times G L_{n_{2}}(k), M_{n_{2}}(k)\right)= \\
& =\bigoplus_{i=0}^{m} H_{i}\left(G L_{n_{1}}(k), M_{n_{1}}(k)\right) \otimes H_{m-i}\left(G L_{n_{2}}(k), k\right) \oplus \\
& \oplus \bigoplus_{j=0}^{m} H_{j}\left(G L_{n_{1}}(k), k\right) \otimes H_{m-j}\left(G L_{n_{2}}(k), M_{n_{2}}(k)\right)= \\
& =H_{m}\left(G L_{n_{1}}(k), M_{n_{1}}(k)\right) \oplus H_{m}\left(G L_{n_{2}}(k), M_{n_{2}}(k)\right) .
\end{aligned}
$$

Moreover after this identification the homomorphism

$$
\begin{aligned}
(\operatorname{diag}(\phi, \psi))_{*}: H_{m}\left(G L_{n}(k), M_{n}(k)\right) & \rightarrow H_{m}\left(A^{*}, A\right)=H_{m}\left(G L_{n_{1}}(k), M_{n_{1}}(k)\right) \oplus \\
& \oplus H_{m}\left(G L_{n_{2}}(k), M_{n_{2}}(k)\right)
\end{aligned}
$$

clearly coincides with $\operatorname{diag}\left(\phi_{*}, \psi_{*}\right)$ and the homomorphisms $H_{m}\left(G L_{n_{i}}(k), M_{n_{i}}(k)\right)$ $\hookrightarrow H_{m}\left(A^{*}, A\right) \rightarrow H_{m}\left(G L_{n_{1}+n_{2}}(k), M_{n_{1}+n_{2}}(k)\right)$ both coincide with the corresponding natural isomorphisms.

The $G L_{n}(k)$-module $M_{n}(k)$ is self dual via the pairing $\alpha \times \beta \mapsto \operatorname{Tr}(\alpha \beta)$. This together with duality in group cohomology gives us a natural isomorphism

$$
H_{m}\left(G L_{n}(k), M_{n}(k)\right)^{\#}=H^{m}\left(G L_{n}(k), M_{n}(k)^{\#}\right)=H^{m}\left(G L_{n}(k), M_{n}(k)\right) .
$$

Thus computation of homology groups $H_{m}\left(G L_{n}(k), M_{n}(k)\right)$ is equivalent to the computation of the corresponding cohomology groups. A beautiful and important theorem due to Cline, Parshall, Scott and van der Kallen [CPSK] relates rational cohomology of a semisimple algebraic group defined over a finite field to the cohomology of the finite group of its rational points. We recall briefly this result.

Let $G / k$ be an affine group scheme and let $M / k$ be a rational $G$-module. In this case we may consider $M$ as a $G(k)$-module as well. Moreover we have natural maps on the cohomology groups $H^{*}(G, M) \rightarrow H^{*}(G(k), M)$, induced by the evident homomorphism of standard complexes $C^{*}(G, M) \rightarrow C^{*}(G(k), M)$. The following property is obvious from definitions

Lemma 7.2. Let $k / k_{0}$ be a finite Galois extension. Assume further that $G=$ $\left(G_{0}\right)_{k}, M=\left(M_{0}\right)_{k}$ are defined over $k_{0}$. In this case we have a natural action of the Galois group $\Gamma=\operatorname{Gal}\left(k / k_{0}\right)$ on $H^{*}(G(k), M)$ and on $H^{*}(G, M)=H^{*}\left(G_{0}, M_{0}\right) \otimes_{k_{0}}$ $k$. Moreover the natural homomorphism $H^{*}(G, M) \rightarrow H^{*}(G(k), M)$ is $\Gamma$-equivariant. In particular the image of $H^{*}\left(G_{0}, M_{0}\right) \subset H^{*}(G, M)$ in $H^{*}(G(k), M)$ is contained in the subgroup of $\Gamma$-invariants. Finally the following diagram commutes for 
any finite extension $k / k_{0}$

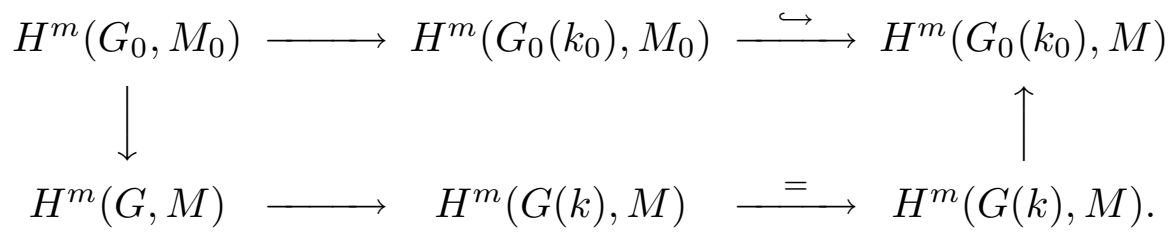

Assume now that $k=\mathbb{F}_{q}\left(q=p^{d}\right)$ is a finite field. Let $G / k$ be an affine group scheme defined over $\mathbb{F}_{p}$ and let $M / k$ be a rational $G$-module defined over $\mathbb{F}_{p}$. In this case the Frobenius twist $M^{(1)}$ may be identified as a $G$-module with $M$ endowed with a new structure of $G$-module via the Frobenius endomorphism $F: G \rightarrow G$. Since the action of $F$ on the group of rational points $G(k)$ is an isomorphism we conclude that $H^{*}\left(G(k), M^{(1)}\right)=H^{*}(G(k), M)$. Thus we get a new homomorphism $H^{*}\left(G, M^{(1)}\right) \rightarrow H^{*}\left(G(k), M^{(1)}\right)=H^{*}(G(k), M)$, making the following diagram commute

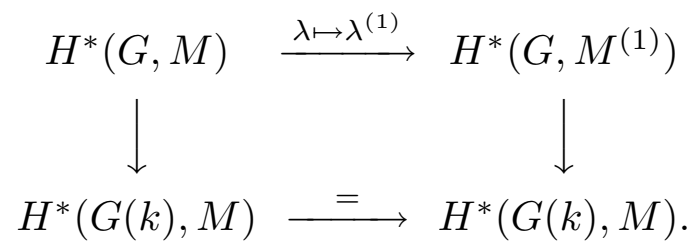

Iterating this procedure we get homomorphisms $H^{*}\left(G, M^{(r)}\right) \rightarrow H^{*}(G(k), M)$ defined for all $r \geq 0$. The main theorem of [CPSK] shows that for a semisimple group scheme $G$ the corresponding homomorphism $H^{*}\left(G, M^{(r)}\right) \rightarrow H^{*}(G(k), M)$ is an isomorphism provided that $r$ and $d$ are big enough. The argument used may be applied without any changes to any reductive group scheme. Here is an explicit formulation of the Main Theorem of [CPSK] in case of $G L_{n}$ (for any weight $\lambda=\left(\lambda_{1}, \ldots, \lambda_{n}\right)$ of $G L_{n}$ we set below $\left.c(\lambda)=\max \left(\left|\lambda_{1}\right|,\left|\lambda_{1}+\lambda_{2}\right|, \ldots,\left|\lambda_{1}+\ldots+\lambda_{n}\right|\right)\right)$.

Theorem 7.3. Let $M$ be a rational $G L_{n, k}$-module defined over $\mathbb{F}_{p}$. Fix an integer $m \geq 0$ and assume that

(1) $r \geq\left[\frac{m-2}{p-1}\right]+1$,

(2) For any weight $\lambda$ of $M$ we have: $d \geq\left[\frac{m-2}{p-1}\right]+\left[\log _{p}(c(\lambda)+1)\right]+3$ (here [] denotes the greatest integer function).

Then the natural homomorphism $H^{i}\left(G L_{n, k}, M^{(r)}\right) \rightarrow H^{i}\left(G L_{n}(k), M\right)$ is an isomorphism for $i \leq m$ and a monomorphism for $i=m+1$.

Note that all weights of the adjoint representation are of the form $\lambda=\epsilon_{s}-\epsilon_{t}$ and hence $c(\lambda)=1$. Thus for the adjoint representation Theorem 7.3 gives

Corollary 7.4. Assume that $r \geq\left[\frac{m-2}{p-1}\right]+1$ and $d \geq\left[\frac{m-2}{p-1}\right]+4$. Then the natural map $H^{i}\left(G L_{n, k}, g l_{n}^{(r)}\right) \rightarrow H^{i}\left(G L_{n}(k), M_{n}(k)\right)$ is an isomorphism for all $i \leq m$.

Let $l \geq 0$ be an integer. Consider its $p$-adic expansion $: l=l_{0}+l_{1} p+\ldots+l_{s} p^{s}$ $\left(0 \leq l_{i}<p, l_{s} \neq 0, s=\left[\log _{p} l\right]\right)$ and denote by $e(l)=e(l, k)$ the image of $\left(e_{1}^{(s)}\right)^{l_{0}} \cdot \ldots$. $\left(e_{s+1}\right)^{l_{s}} \in H^{2 l}\left(G L_{n, k}, g l_{n}^{(s+1)}\right)$ in

$$
H^{2 l}\left(G L_{n}(k), M_{n}(k)\right)=H o m_{k}\left(H_{2 l}\left(G L_{n}(k), M_{n}(k)\right), k\right) .
$$

In the next Lemma we list some of the properties of the cohomology classes $e(l)$. 


\section{Lemma 7.5.}

(1) The homomorphism $e(l): H_{2 l}\left(G L_{n}(k), M_{n}(k)\right) \rightarrow k$ is $G a l\left(k / \mathbb{F}_{p}\right)$-equivariant.

(2) Let $k \subset k^{\prime}$ be an extension of finite fields. Then the following diagram commutes

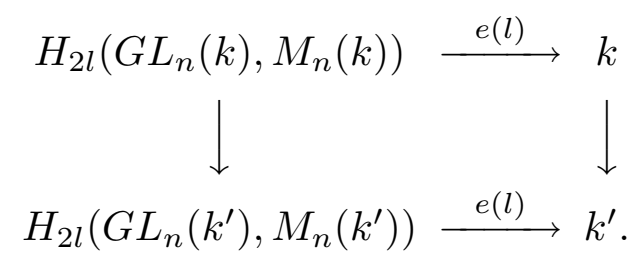

(3) Assume that $n \leq n^{\prime}$. Then the following diagram commutes

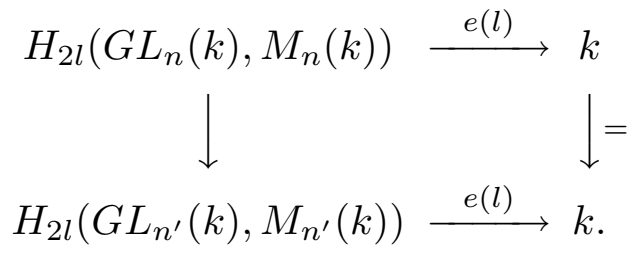

Proof. The first two statements follow from Lemma 7.2 and the last one from Lemma 6.1.

Theorem 7.6. Let $k=\mathbb{F}_{q}\left(q=p^{d}\right)$ be a finite field. Assume that $n \geq N(m)$. Then $H_{m}\left(G L_{n}(k), M_{n}(k)\right)=0$ if $m$ is odd. On the other hand if $m=2 l$ is even, then the homomorphism $e(l): H_{m}\left(G L_{n}(k), M_{n}(k)\right) \rightarrow k$ is an isomorphism.

Proof. Assume first that $d \geq\left[\frac{m-2}{p-1}\right]+4$. Set $r=\left[\frac{m-2}{p-1}\right]+1, s=\left[\log _{p}(m / 2)\right], N=$ $\max \left(n, p^{r}\right)$. Corollary 7.4 shows that $H_{m}\left(G L_{N}(k), M_{N}(k)\right)^{\#}=$ $H^{m}\left(G L_{N}(k), M_{N}(k)\right)=H^{m}\left(G L_{N, k}, g l_{N}^{(r)}\right)$. Furthermore we conclude from 4.11 that this group is trivial for $m$ odd. Assume now that $m=2 l$. A trivial verification shows that $l<p^{r}$ and according to $4.11 H^{m}\left(G L_{N, k}, g l_{N}^{(r)}\right)$ is one-dimensional spanned by $\left(\left(e_{1}^{(s)}\right)^{l_{0}} \cdot \ldots \cdot\left(e_{s+1}\right)^{l_{s}}\right)^{(r-1-s)}$. Commutativity of the diagram $(7 . .2 .1)$ shows finally that the image of this basis element in $H^{m}\left(G L_{N}(k), M_{N}(k)\right)$ is equal to $e(l)$. We conclude the proof in this case using Lemma $7.5(3)$.

For arbitrary $k$ we may proceede as follows. Choose $d^{\prime}$ prime to $p$ such that $d \cdot d^{\prime} \geq\left[\frac{m-2}{p-1}\right]+4$ and let $k^{\prime}$ be the extension of $k$ of degree $d^{\prime}$. We may apply the already proved part of the statement to $k^{\prime}$. Proposition 7.1 shows that the $\operatorname{map} H_{m}\left(G L_{n}(k), M_{n}(k)\right) \rightarrow H_{m}\left(G L_{n}\left(k^{\prime}\right), M_{n}\left(k^{\prime}\right)\right)$ is injective. This settles immediately the case of the odd $m$. Assume finally that $m=2 l$. Commutativity of the diagram

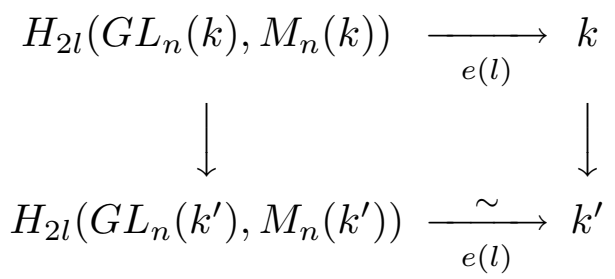

together with (7.1) implies that the map $e(l): H_{2 l}\left(G L_{n}(k), M_{n}(k)\right) \rightarrow k$ is injective. It suffices to show now that the corresponding homology group is non 
zero. Denote by $\Gamma$ the Galois group $\operatorname{Gal}\left(k^{\prime} / \mathbb{F}_{p}\right)$. Lemma $7.5(1)$ shows that $e(l)$ : $H_{2 l}\left(G L_{n}\left(k^{\prime}\right), M_{n}\left(k^{\prime}\right)\right) \stackrel{\sim}{\longrightarrow} k^{\prime}$ is an isomorphism of $\Gamma$-modules. Proposition 7.1 shows now that the composition

$$
k^{\prime}=H_{2 l}\left(G L\left(k^{\prime}\right), M\left(k^{\prime}\right)\right) \stackrel{T r_{k^{\prime} / k}}{\longrightarrow} H_{2 l}(G L(k), M(k)) \rightarrow H_{2 l}\left(G L\left(k^{\prime}\right), M\left(k^{\prime}\right)\right)=k^{\prime}
$$

coincides with the usual trace map $k^{\prime} \stackrel{T r_{k^{\prime} / k}}{\longrightarrow} k \hookrightarrow k^{\prime}$. Since this composition is non trivial we conclude that $H_{2 l}(G L(k), M(k)) \neq 0$.

\section{§8. EXPLICIT RESOLUTIONS IN CHARACTERISTIC 2.}

In this section $k$ denotes a field of characteristic 2. As recognized in [FLS], this case lends itself to specific resolutions. A construction used in [FLS] gives an injective resolution of the functor $S^{m(1)}$. We develop this construction further and produce canonical injective resolutions of all functors $S^{m(r)}$. Using these resolutions for the computation of Ext-groups we get explicit extensions representing the classes $e_{r}$. One interesting point is that the above resolutions are finite so that the functors $S^{m(r)}$ have finite injective dimension (equal to $2 m\left(2^{r}-1\right)$ ). We don't know if the same holds for $p \neq 2$.

For any $V \in \mathcal{V}$ set $B^{j, i}(V)=S^{j}(V) \otimes S^{i}(V), B(V)=B^{*, *}(V)=\bigoplus_{j, i=0}^{\infty} B^{j, i}(V)$. We shall refer to $i$ as a cohomological degree and to $i+j$ as a total degree. We shall also use notation $B_{n}^{i}(V)=B^{n-i, i}(V)$. $B^{*, *}(V)$ has a natural structure of a bigraded commutative algebra. We endow this algebra with a differential $d=d_{1}$ defined in terms of the product and coproduct operations as follows:

$$
\begin{aligned}
& d: B^{j, i}(V)=S^{j}(V) \otimes S^{i}(V) \stackrel{\text { comult } \otimes 1}{\longrightarrow}\left(S^{j-1}(V) \otimes V\right) \otimes S^{i}(V)= \\
& =S^{j-1}(V) \otimes\left(V \otimes S^{i}(V)\right) \stackrel{1 \otimes \mathrm{mult}}{\longrightarrow} S^{j-1}(V) \otimes S^{i+1}(V)=B^{j-1, i+1}(V) .
\end{aligned}
$$

Note that $d$ increases the cohomological degree by 1 and preserves the total degree so that $B^{*, *}(V)$ is a direct sum of its homogenous (with respect to total degree) components $B_{n}^{*}(V)$. Furthermore $d$ is a derivation and hence $H^{*}(B(V))$ has a natural structure of a bigraded commutative algebra. Finally we have a natural homomorphism

$$
V^{(1)} \rightarrow H^{0}\left(B_{2}^{*}(V)\right): \quad v^{(1)} \mapsto \text { homology class of the cycle } v^{2} \in S^{2}(V)=B_{2}^{0}(V)
$$

Theorem 8.1[FLS]. The induced map of graded algebras $S^{*}\left(V^{(1)}(2)\right) \rightarrow$ $\rightarrow H^{*}(B(V))$ is an isomorphism. Here as before the index 2 in parantheses indicates the degree in which $V^{(1)}$ is positioned and the cohomology algebra is graded by means of total degree.

Theorem 8.1 shows that the complex $B_{n}^{*}(V)$ is acyclic if $n$ is odd whereas the complex $B_{2 m}^{*}(V)$ is a resolution of $S^{m}\left(V^{(1)}\right)$. The above construction is clearly functorial in $V$ so that we may, in particular, consider $B_{2 m}^{*}$ as an injective resolution of the polynomial functor $S^{m(1)}$. We want to show that iterating the above construction provides canonical resolutions of all functors of the form $S^{m(r)}$. 
The differential $d=d_{1}$ is one of a family of differentials $d_{l}(l=1,2, \ldots)$ defined via the formula

$$
\begin{aligned}
& d_{l}: B^{j, i}(V)=S^{j}(V) \otimes S^{i}(V) \stackrel{\text { comult } \otimes 1}{\longrightarrow}\left(S^{j-l}(V) \otimes S^{l}(V)\right) \otimes S^{i}(V)= \\
& \quad=S^{j-l}(V) \otimes\left(S^{l}(V) \otimes S^{i}(V)\right) \stackrel{1 \otimes \text { mult }}{\longrightarrow} S^{j-l}(V) \otimes S^{i+l}(V)=B^{j-l, i+l}(V) .
\end{aligned}
$$

The differentials $d_{l}$ have the following properties:

(8.2.1) $d_{l}$ increases the cohomological degree by $l$ and preserves the total degree.

(8.2.2) If we define $d_{0}$ to be the identity map then for any $x, y \in B^{*, *}(V)$ the following relation holds

$$
d_{l}(x y)=\sum_{t=0}^{l} d_{t}(x) d_{l-t}(y)
$$

(8.2.3) If $l$ is odd then $d_{l}\left(x^{2}\right)=0$ for any $x \in B(V)$. On the other hand $d_{2 j}\left(x^{2}\right)=$ $d_{j}(x)^{2}$.

(8.2.4) $d_{l}\left(x^{2^{r}}\right)=0$ unless $l \equiv 0 \bmod 2^{r}$.

Assume now that $r \geq 1$ is any integer. Consider the commutative algebra

$$
B(r)(V)=\underbrace{S^{*}(V) \otimes \ldots \otimes S^{*}(V)}_{2^{r}}=S^{*}(\underbrace{V \oplus \ldots \oplus V}_{2^{r}})
$$

We index the factors (summands) in the above formula by integers varying from 0 to $2^{r}-1$ and for any nonnegative integer $i$ (and any $s \geq 0$ ) we define $\epsilon_{s}(i)$ to be the $s$-th digit in the 2-adic expansion of $i$. For each $j$ and each $s$ with $0 \leq s \leq r-1$, we define a differential $d_{j}^{s}$ on the above algebra as follows. Split summands in the above direct sum in two groups depending on the $s$-th digit of the index of the summand. Thus $\underbrace{V \oplus \ldots \oplus V}_{2^{r-1}}=W^{s} \oplus W^{s}$, where the first (resp. the second) $W^{s}$ is the direct sum of summands corresponding to indices $i$ with $\epsilon_{s}(i)=0\left(\operatorname{resp} . \epsilon_{s}(i)=1\right)$ and two copies of $W^{s}$ are identified by identifying the summands whose indices differ only in the $s$-th digit. This gives us a natural isomorphism $B(r)(V)=B\left(W^{s}\right)$ and we define $d_{j}^{s}$ to be the differential $d_{j}$ corresponding to the algebra $B\left(W^{s}\right)$.

Lemma 8.3. The differentials $d_{j}^{s}$ and $d_{l}^{t}$ commute for all $j, l$ and all $0 \leq s, t \leq r-1$.

Proof. Fix $s$ and $t$. Assume that elements $x, y \in B(r)(V)$ have the property that $\left[d_{j}^{s}, d_{l}^{t}\right](x)=\left[d_{j}^{s}, d_{l}^{t}\right](y)=0$ for all $j$ and $l$ then one shows immediately, using the property $(8.2 .2)$ above that $\left[d_{j}^{s}, d_{l}^{t}\right](x \cdot y)=0$ for all $j, l$. Thus it suffices to check the formula in question for a set of generators of the algebra $B(r)(V)$. We will be using the following evident set of generators:

$$
x_{i}(v)=1 \otimes \ldots \otimes \underset{\text { in position } i}{v} \otimes \ldots \otimes 1
$$

The validity of the relation $\left[d_{j}^{s}, d_{l}^{t}\right](x)=0$ for these generators follows immediately from the following formula: 


$$
d_{j}^{s}\left(x_{i}(v)\right)=\left\{\begin{aligned}
0 & \text { if } j>1 \\
0 & \text { if } j=1, \text { but } \epsilon_{s}(i)=1 \\
x_{i+2^{s}}(v) & \text { if } j=1 \text { and } \epsilon_{s}(i)=0 \\
x_{i}(v) & \text { if } j=0 .
\end{aligned}\right.
$$

Lemma 8.3 guarantees that $d=d(r)=d_{1}^{0}+d_{2}^{1}+\ldots+d_{2^{r-1}}^{r-1}$ is a differential. We intend to compute the homology of $B(r)(V)$ with respect to this differential. Note first of all that all differentials $d_{j}^{s}$ (and hence also $d$ ) preserve the total degree, so that the vector space $H^{*}((B(r)(V), d))$ is naturally graded. Furthermore property (8.2.4) shows that the image of $S^{*}\left(V^{(r)}\left(2^{r}\right)\right)$ under the embeding

$$
S^{*}\left(V^{(r)}\left(2^{r}\right)\right) \hookrightarrow S^{*}(V) \hookrightarrow B(r)(V),
$$

where the first arrow is induced by the standard embeding $V^{(r)} \hookrightarrow S^{2^{r}}(V): \quad v \mapsto$ $v^{2^{r}}$ and the second arrow identifies $S^{*}(V)$ with the first tensor factor of $B(r)(V)$ (i.e. the one corresponding to index 0 ), is contained in the subspace of cycles (relative to all $d_{i}^{2^{i}} \quad 0 \leq i \leq r-1$ and hence also relative to $d$ ). This gives us a natural homomorphism of graded vector spaces

$$
S^{*}\left(V^{(r)}\left(2^{r}\right)\right) \rightarrow H^{*}((B(r)(V), d)) .
$$

Theorem 8.4. The above homomorphism $S^{*}\left(V^{(r)}\left(2^{r}\right)\right) \rightarrow H^{*}((B(r)(V), d))$ is an isomorphism.

Proof. We proceed by induction on $r$. The case $r=1$ is covered by Theorem 8.1. Assuming now that the statement is true for $r-1$ we proceed to show that it holds for $r$ as well. For a polyhomogenous element $x \in S^{m_{0}}(V) \otimes \ldots \otimes S^{m_{2^{r}-1}}(V)$ set

$$
\operatorname{deg}^{s}(x)=\sum_{\epsilon_{s}(i)=1} m_{i}
$$

Note that the differential $d_{j}^{s}$ takes polyhomogenous elements to polyhomogenous, increases $d e g^{s}$ by $j$ and does not change $d e g^{t}$ with $t \neq s$. We define differentials $d^{\prime}, d^{\prime \prime}$ and degrees $d e g^{\prime}$, deg $g^{\prime \prime}$ via the formulae

$$
\begin{gathered}
d^{\prime}=\sum_{s=0}^{r-2} d_{2^{s}}^{s} ; \quad d^{\prime \prime}=d_{2^{r-1}}^{r-1} \\
d e g^{\prime}=\sum_{s=0}^{r-2} 2^{r-s-1} \cdot d e g^{s} ; \quad d e g^{\prime \prime}=d e g^{r-1}
\end{gathered}
$$

Thus $d^{\prime}$ increases $d e g^{\prime}$ by $2^{r-1}$ and does not change $d e g^{\prime \prime}$, whereas $d^{\prime \prime}$ increases $d e g^{\prime \prime}$ by $2^{r-1}$ and does not change $d e g^{\prime}$. Since $d=d^{\prime}+d^{\prime \prime}$ we may consider $B(r)(V)$ as a bicomplex with respect to the above degrees. Consider further the first spectral sequence of this bicomplex. The $E_{1}$-term of this spectral sequence coincides with homology of the complex $\left(B(r)(V), d^{\prime}\right)$. To compute the homology of this complex 
we proceed as follows. Set $U=V \oplus V$. Note that for any $0 \leq i \leq 2^{r-1}-1$ the summands with numbers $i$ and $i+2^{r-1}$ appear in the same group when we split the summands in two groups depending on the $s$-th digit with $0 \leq s \leq r-2$. Thus if we replace two copies of $V$ with these numbers by one copy of $U$ (placed in position $i$ ) we get an isomorphism $S^{*}(\underbrace{V \oplus \ldots \oplus V}_{2^{r}})=S^{*}(\underbrace{U \oplus \ldots \oplus U}_{2^{r-1}})$ and this isomorphism is compatible with differentials $d_{2^{s}}^{s}(0 \leq s \leq r-2)$. This shows that $\left(B(r)(V), d^{\prime}\right)=$ $(B(r-1)(U), d(r-1))$. The induction hypotheseis implies that the above discussed embeding $\left(S^{*}\left(U^{(r-1)}\left(2^{r-1}\right)\right), 0\right) \hookrightarrow(B(r-1)(U), d(r-1))=\left(B(r)(V), d^{\prime}\right)$ is a quasiisomorphism. Note further that the differential $d^{\prime \prime}$ preserves $S^{*}\left(U^{(r-1)}\left(2^{r-1}\right)\right)$. Moreover the latter vector space may be identified (up to multiplication of all degrees by $2^{r-1}$ ) with $B\left(V^{(r-1)}\right)$ and the restriction of $d^{\prime \prime}$ to this vector space coincides with the corresponding differential $d$. The standard spectral sequence argument shows that the embeding (where in the complex on the left all degrees are multiplied by $2^{r-1}$ )

$$
\left(B\left(V^{(r-1)}\right), d\right) \hookrightarrow(B(r)(V), d)
$$

is a quasi-isomorphism. Thus it suffices to use the Theorem 8.1 to end the proof.

Theorem 8.4 gives injective resolutions for functors $S^{m(r)}$. To be more specific, set $d e g=d e g^{\prime}+d e g^{\prime \prime}$ (in notations of the above proof). Note that deg is always non negative and the only terms of degree zero are of the form $S^{m_{0}} \otimes 1 \otimes \ldots \otimes 1$, so that, in particular the image of $S^{*}\left(I^{(r)}\left(2^{r}\right)\right)$ in $B(r)$ consists of elements of degree zero. Furthermore the differential $d$ of the complex $B(r)$ increases $d e g$ by $2^{r-1}$ (and does not change the total degree). We derive from Theorem 8.4 the following injective resolution of the functor $S^{m(r)}$ :

$$
0 \rightarrow S^{m(r)} \rightarrow C^{0} \rightarrow C^{1} \rightarrow \ldots \rightarrow C^{n} \rightarrow \ldots
$$

Here $C^{n}(V)$ is the direct sum of those polyhomogenous components of $B(r)(V)$ which have deg equal to $n 2^{r-1}$ and total degree equal to $m 2^{r}$.

It seems useful to have a more explicit formula for the degree deg. To give such a formula we introduce the following notation: for any $0 \leq i \leq 2^{r}-1$ we let $0 \leq \bar{i} \leq 2^{r}-1$ denote the integer having the same digits in the 2-adic expansion, but going in the opposite order. One checks easily the following formula:

$$
\operatorname{deg} S^{m_{0}} \otimes \ldots \otimes S^{m_{2}-1}=\sum_{i=0}^{2^{r}-1} \bar{i} \cdot m_{i}
$$

Corollary 8.6. inj $\operatorname{dim} S^{m(r)}=2 m\left(2^{r}-1\right)$.

Proof. Consider the injective resolution (8.5.1) of the functor $S^{m(r)}$. Assume that $C^{n} \neq 0$. This means that there exists $\left(m_{0}, \ldots, m_{2^{r}-1}\right)$ such that $m_{0}+\ldots+m_{2^{r}-1}=$ $m 2^{r}, \quad \sum_{i=0}^{2^{r}-1} \bar{i} \cdot m_{i}=n 2^{r-1}$. From these equations we get:

$$
n=\frac{\sum_{i=0}^{2^{r}-1} \bar{i} m_{i}}{2^{r-1}} \leq \frac{\left(2^{r}-1\right) \sum_{i=0}^{2^{r}-1} m_{i}}{2^{r-1}}=2 m\left(2^{r}-1\right) .
$$


Thus the length of the resolution (8.5.1) equals $2 m\left(2^{r}-1\right)$ and hence $i n j \operatorname{dim} S^{m(r)}$ $\leq 2 m\left(2^{r}-1\right)$. To prove the opposite inequality we compute, using the resolution (8.5.1), the Ext-groups $\operatorname{Ext}_{\mathcal{P}}^{*}\left(\Gamma^{m(r)}, S^{m(r)}\right)$. The statement dual to Corollary 2.12 shows that for any $\left(m_{0}, \ldots, m_{2^{r}-1}\right)$ with $m_{0}+\ldots+m_{2^{r}-1}=m 2^{r}$ we have:

$$
\operatorname{Hom}_{\mathcal{P}}\left(\Gamma^{m(r)}, S^{m_{0}} \otimes \ldots \otimes S^{m_{2}{ }^{2}-1}\right)=\left(\Gamma^{m}\left(\left(k^{2^{r}}\right)^{(r)}\right)^{m_{0}, \ldots, m_{2^{r}-1}}\right)^{\#} .
$$

Furthermore this space is one dimensional if $m_{i} \equiv 0 \bmod 2^{r}$ for all $i$ and is trivial otherwise. This shows immediately that $\operatorname{Hom}_{\mathcal{P}}\left(\Gamma^{m(r)}, C^{n}\right)=0$ if $n$ is odd. On the other hand $C^{2 m\left(2^{r}-1\right)}=1 \otimes \ldots \otimes 1 \otimes S^{m 2^{r}} \cong S^{m 2^{r}}$ and hence $\operatorname{Hom}_{\mathcal{P}}\left(\Gamma^{m(r)}, C^{2 m\left(2^{r}-1\right)}\right)=k$. This computation shows that $\operatorname{Ext}_{\mathcal{P}}^{2 m\left(2^{r}-1\right)}\left(\Gamma^{m(r)}, S^{m(r)}\right)=k \neq 0$ and hence $i n j \operatorname{dim} S^{m(r)} \geq 2 m\left(2^{r}-1\right)$.

Consider finally the special case $m=1$. Corollary 8.6 shows that $i n j \operatorname{dim} I^{(r)}=$ $2^{r+1}-2$. Furthermore, repeating the argument used in the proof of the Theorem 8.6 we see that for any $\left(m_{0}, \ldots, m_{2^{r}-1}\right)$ with $\sum_{i=0}^{2^{r}-1} m_{i}=2^{r}$ we have $\operatorname{Hom}_{\mathcal{P}}\left(I^{(r)}, S^{m_{0}} \otimes\right.$ $\left.\ldots \otimes S^{m_{2^{r}-1}}\right)=0$ unless all $m_{i}$ but one are zero. Since $\operatorname{deg}\left(1 \otimes \ldots \otimes S_{j}^{2^{r}} \otimes \ldots \otimes 1\right)=2 \bar{j}$ we conclude that

$$
\operatorname{Hom}_{\mathcal{P}}\left(I^{(r)}, C^{n}\right)=\left\{\begin{array}{l}
k \text { if } n \leq 2^{r+1}-2, n \equiv 0 \bmod 2 \\
0 \text { otherwise }
\end{array}\right.
$$

Thus the complex computing $\operatorname{Ext}_{\mathcal{P}}^{*}\left(I^{(r)}, I^{(r)}\right)$ looks as follows:

$$
k \rightarrow 0 \rightarrow k \rightarrow 0 \rightarrow \ldots \rightarrow \underset{2^{r+1}-2}{k}
$$

Since the cohomology class $e_{r} \in \operatorname{Ext}_{\mathcal{P}}^{2^{r}}\left(I^{(r)}, I^{(r)}\right)$ is defined over the prime field $\mathbb{F}_{2}$ we conclude finally that $e_{r}$ corresponds to the natutal embeding

$$
I^{(r)} \hookrightarrow S^{2^{r}}=1 \otimes S^{2^{r}} \otimes \ldots \otimes 1 \subset C^{2^{r}}
$$

The above remarks show that to construct an explicit extension corresponding to $e_{r}$ we have to proceed as follows. Let $Z^{2^{r}} \subset C^{2^{r}}$ be the subspace of cocycles. Truncating the resolution $C^{\bullet}$ at the level $2^{r}$ we get an extension

$$
0 \rightarrow I^{(r)} \rightarrow C^{0} \rightarrow \ldots \rightarrow C^{2^{r}-1} \rightarrow Z^{2^{r}} \rightarrow 0
$$

Since $\operatorname{Hom}_{\mathcal{P}}\left(I^{(r)}, C^{2^{r}+1}\right)=0$ we conclude that the image of $I^{(r)}$ in $C^{2^{r}}$ is contained in $Z^{2^{r}}$. Pulling back the above extension via the homomorphism $I^{(r)} \hookrightarrow Z^{2^{r}}$ we get the extension defining the class $e_{r}$.

\section{REFERENCES}

[B] M. Bökstedt, The topological Hochschild homology of $\mathbf{Z}$ and $\mathbf{Z} / p$, preprint.

[C] P. Cartier, Une nouvelle opération sur les formes différentielles, C.-R. Acad. Sci Paris 244 (1957), 426-428.

[CPSK] E. Cline, B. Parshall, L. Scott, and W. van der Kallen, Rational and generic cohomology, Inventiones Math. 39 (1977), 143-163. 
[D] S. Donkin, On Schur Algebras and related algebra, I, J. of Algebra 104, no. 2 (1986), 310-328.

[DW] W. Dwyer, Twisted homological stability for general lienar groups, Annals of Math. 111 (1980), 239-251.

[E] L. Evens, The cohomology ring of a finite group, Trans. A.M.S. 101 (1961), 224-239.

[FLS] V. Franjou, J. Lannes, and L. Schwartz, Autor de la cohomologie de MacLane des corps finis, Inventiones Math 115 (1994), 513-538.

[FP1] E. Friedlander and B. Parshall, On the cohomology of algebraic and related finite groups, Inventiones Math. 74 (1983), 85-117.

[FP2] E. Friedlander and B. Parshall, Cohomology of infinitesimal and discrete groups, Mathematische Annalen 273 (1986), 353-374.

[F] E.Friedlander, A canonical filtration for certain rational modules, Math. Zeitschrift 188 (1985), 433-438.

[G] E.S. Golod, The cohomology ring of a finite p-group, Doklady 125 (1959), 703-706.

[J] J. Jantzen, Representations of algebraic groups (Academic Press, ed.), 1987.

[JP] M. Jibladze and T. Pirashvili, Cohomology of algebraic theories, J. of Algebra 137 (1991), 253-296.

[M] J.P. May, The cohomology of restricted Lie algebras and Hopf algebras, J. of Algebra 3 (1966), 123-146.

[Q] D. Quillen On the cohomology and K-theory of the general linear group over a finite field, Annals of Math. 96 (1972), 552-586.

[V] B. Venkov, Cohomology algebras for some classifying spaces, Dokl. Akad. Nauk. SSSR 127 (1959), 943-944.

[W] W. Waterhouse, Introduction to Affine Group Schemes (Spring-Verlag, ed.), 1979. 